\title{
Helicopter Tail Rotor Orthogonal Blade Vortex Interaction
}

\author{
F.N. Coton ${ }^{\mathrm{a}, *}$, J.S. Marshall ${ }^{\mathrm{b}}$, R.A.M ${ }^{\mathrm{c}}$ D. Galbraith ${ }^{\mathrm{a}}$, R.B. Green ${ }^{\mathrm{a}}$ \\ a Department of Aerospace Engineering, University of Glasgow, Glasgow, G12 8QQ, \\ U.K. \\ ${ }^{\mathrm{b}}$ Department of Mechanical and Industrial Engineering and IIHR - Hydroscience and \\ Engineering, The University of Iowa, Iowa City, IA 52242, U.S.
}

\begin{abstract}
The aerodynamic operating environment of the helicopter is particularly complex and, to some extent, dominated by the vortices trailed from the main and tail rotors. These vortices not only determine the form of the induced flow field but also interact with each other and with elements of the physical structure of the flight vehicle. Such interactions can have implications in terms of structural vibration, noise generation and flight performance. In this paper, the interaction of main rotor vortices with the helicopter tail rotor is considered and, in particular, the limiting case of the orthogonal interaction. The significance of the topic is introduced by highlighting the operational issues for helicopters arising from tail rotor interactions. The basic phenomenon is then described before experimental studies of the interaction are presented. Progress in numerical modelling is then considered and, finally, the prospects for future research in the area are discussed.
\end{abstract}

\footnotetext{
* Corresponding Author. Tel.: +44 141330 4305, Fax.: +44 141330 5560,

E-Mail.: f.coton@aero.gla.ac.uk
} 


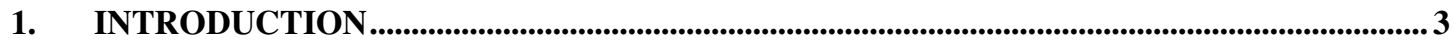

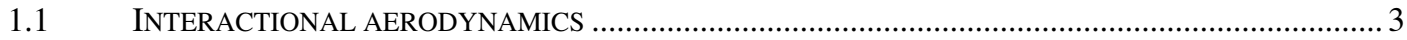

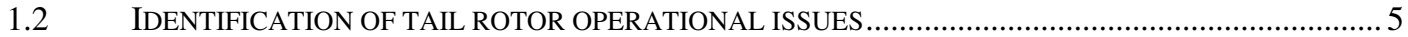

1.3 BASIC FEATURES OF ORTHOGONAL BLADE VORTEX INTERACTION .......................................... 6

2. EXPERIMENTAL STUDIES OF ORTHOGONAL BLADE VORTEX INTERACTION ...... 7

2.1 EARLY EXPERIMENTAL STUDIES OF ORTHOGONAL BLADE VORTEX INTERACTION..................... 8

2.2 EXPERIMENTAL STUDIES AT THE UNIVERSITY OF IOWA........................................................ 10

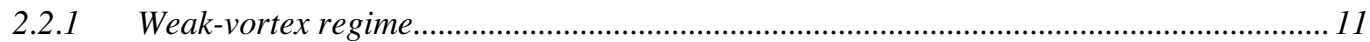

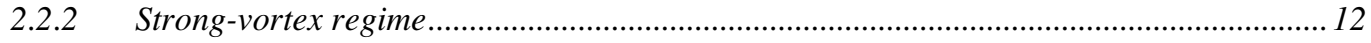

2.3 EXPERIMENTAL STUDIES AT THE UNIVERSITY OF GLASGOW ................................................ 13

2.3.1 Orthogonal interaction on a zero-loaded blade ............................................................... 18

2.3.2 Effect of vortex approach angle ....................................................................................... 20

2.3.3 Orthogonal interaction on a loaded blade ......................................................................... 21

2.3.4 The effect of vortex pre-cut on the interaction response ................................................... 22

2.3.5 The effect of sub-critical axial flow on the interaction response .......................................22

2.3.6 Interaction measurements on a model tail rotor............................................................. 23

3. NUMERICAL MODELLING OF ORTHOGONAL BLADE VORTEX INTERACTION... 24

3.1 FLUID DYNAMIC SIMULATIONS OF ORTHOGONAL BLADE VORTEX INTERACTION..................... 25

3.1.1 Inviscid flow modelling of orthogonal blade vortex interaction ......................................... 25

3.1.2 Viscous flow modelling of orthogonal blade vortex interaction.......................................22

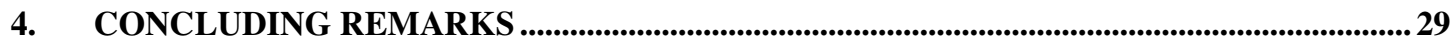

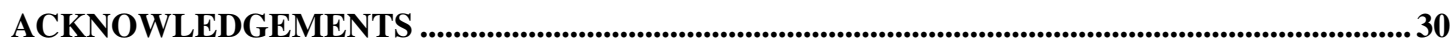

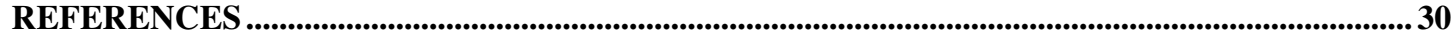




\section{Introduction}

Vortices pervade our entire environment [1] and have many forms. The type considered in this paper are those that trail from the tips of lifting surfaces and, in particular, the vortices produced by the main rotors of helicopters [2-4]. Of interest here is the manner in which these vortices interact with the helicopter tail rotor. When a vortex passes close to or impacts with a helicopter rotor blade, the event is termed a Blade Vortex Interaction (BVI). Tail rotor BVIs occur for a wide range of flight conditions and can, therefore, take many geometrical forms. In addition, not only do the main rotor vortices influence the tail rotor performance but, in some cases, particularly near hover conditions, the tail rotor influences the main rotor wake [5] development. These mutual effects complicate the flow field and so make the problem of both assessing and modelling the flow very difficult indeed. For forward level flight at moderate speed, the main rotor wake structure bears some similarity to the simplistic prescribed representation [6] shown in Fig. 1. In this figure it may be seen that as the wake structure passes the location where the tail rotor would be it would be nearly orthogonal to the tail rotor disk plane, albeit the overall field is convoluted. The pure orthogonal blade vortex interaction (OBVI) is, therefore, an important and limiting interaction case and, as such, has been the subject of a considerable volume of research in recent years. This type of interaction provides the focus for this review.

\subsection{Interactional aerodynamics}

It could be thought that, given the complexity of the aerodynamic environment and the important role of the tail rotor, there would have been intensive research to provide an adequate appreciation of the phenomena involved in support of detailed modelling. The contrary is in fact the case and the tail rotor's development and design has been an almost heuristically iterative process [7]. This is in stark contrast to the effort that has been expended on understanding main-rotor interactional aerodynamics and that has fuelled the development of advanced modelling techniques for loads, noise, vibration and Higher Harmonic Control. Today, much effort continues to be directed towards both understanding and modelling main rotor wake vortices and their interactions.

Main rotor blade vortex interactions, as shown in Fig. 2., can take a variety of forms that can be generally categorized as oblique, parallel and perpendicular/grazing $[8,9]$. When interactions occur, they produce a truly unsteady and impulsive flow field resulting in high levels of vibration and radiated noise [10].

It would seem to be axiomatic that, to develop 'proper' modelling of a particular interaction, a detailed knowledge and understanding of the interaction is desirable. To this end experiments on both parallel and oblique BVIs (Fig. 3) on the main rotor were performed as early as 1969 [11,12] and more comprehensively in later years [1315]. The first experiments were pioneering whilst the latter, with the availability of more capable instrumentation, were more able to aid identification of the dominant phenomena involved in the interactions. For the parallel case the chordwise blade surface pressure response to the main interaction exhibited a primary high-speed wave 
followed by a much slower one [13]. This was further elucidated [14] as corresponding to the collapse of the blade pressure distribution close to the moment of impact followed by the passage of the vortex across the chord (Fig. 4). Figure 4 depicts the temporal variation in upper-surface pressure distribution for a 'Head On' impact. Time runs from the rear to the front of the plot and the pressure profiles are plotted as $-\mathrm{Cp}$ against chordwise position on the vertical and horizontal axes respectively. As the vortex approaches the blade there is an obvious increase in the peak suction with a full profile behind. At impact, the entire pressure profile collapses quickly; 'the primary wave'. This is followed by the secondary wave corresponding to the much slower traverse of the vortex fragment across the chord. The resulting overall aerodynamic coefficients reflected these findings and were consistent with numerical predictions $[14,15]$.

In the case of main rotor perpendicular interactions, the axis of the tip vortex is parallel to the blade chord and perpendicular to the blade span. When this type of interaction occurs, a rotor blade is essentially passing through the turbulent wake of a preceding blade. For this reason, the interaction is often termed a Blade Wake Interaction (BWI) and is associated with the production of broadband noise. This is in stark contrast to the strong impulsive noise generation associated with BVI events. It is, however, important to realise that the same tip vortex may produce a series of BWI and BVI events as it migrates downstream. The effect of a BWI on the tip vortex structure may, therefore, influence the noise generated by subsequent BVIs.

Wittmer, Devenport and their co-workers [16-18] recognised that, whilst initial studies of BWI had focussed on the aerodynamic response of the blade during the interaction, the response of the vortex was potentially more important. They conducted a series of simplified experiments to examine the structure of a trailing tip vortex in the aftermath of a perpendicular interaction. It was established that, for cases where the vortex core passed within 0.4 chord lengths of the blade, the effect of the interaction was to both increase the vortex core size and decrease its circulation. Interestingly, it was suggested that the primary cause of this change was the interaction of the vortex with the blade wake rather than the blade itself. The interaction also produced a significant region of turbulent flow around the vortex that is consistent with the high levels of BWI noise produced by actual helicopter rotors.

Another form of interaction that is of importance to helicopters is the interaction of the main rotor wake with either the main fuselage or the tail boom. This interaction is interesting because its basic geometry can, under certain conditions, be similar to OBVI experienced by tail rotors. Both experimental [19-21] and computational studies [22] of this phenomenon have been conducted and significant advances have been made in the understanding of it. In particular, it has been established that the tip vortices deform significantly prior to impact on the vehicle surface and that the form of the pressure response at the surface varies considerably depending on relative location of the measurement point with respect to the point of impingement of the vortex core. Differences in response have also been observed between measurements made on the top and side surfaces of the airframe. The observed deformation of the vortex structure is consistent with the relatively high ratio of the body thickness to vortex core size. In the case of a tail rotor blade, this ratio is much smaller and the deformation of the vortex prior to impact with the blade is almost negligible. 
Although there have been relatively few studies of the detailed aerodynamics of helicopter tail rotors, much work was done on assessing the overall performance of particular tail rotors. These studies initially grew out of the need to resolve documented problems associated with specific helicopters. At first, the problems identified were primarily performance related and were, to some extent, overcome by manufacturers and operators in a pragmatic manner. Surveys of the state of the art $[23,24]$ tended to concentrate on the symptoms of the problems rather than their causality, albeit the interaction of the tail rotor with the main rotor wake was known to be a significant contributor to the observed handling deficiencies of particular designs or, more generally, in specific flight states.

An example of this is quartering flight at, say, Green $60^{\circ}$ i.e. a heading of sixty degrees to starboard. In this flight state, the main rotor often acts like a wing with the main rotor wake rolling up into two distinct vortices [25]. If, for example, the starboard vortex were to line up with a blade-top-forward tail rotor, then yaw control could be severely compromised by a lack of tail rotor thrust, as indicated in Fig 5. which depicts the Lynx yaw control at all headings. For this aircraft, the obvious lack of control at 'Green 60' was moderated by a tail rotor rotation reversal.

Apart from performance, the radiated noise from helicopters, both civil and military, has always been a cause for concern. In fact, the measurement and analysis of the radiated noise was the subject of a considerable body of early research [26-28] in which, much of the work was simply to assess methods of noise reduction and achieving certification [29]. In many cases, there has been considerable success in reducing acoustic emissions. For example, when a Westland Lynx was fitted with a new tail rotor, rotating top blade aft and with new aerofoils, a reduction in the acoustic signature [30] was observed. It has also been possible to identify the influence of operating parameters, such as the tail rotor thrust setting, on noise levels [30,31]. A comprehensive review [27] of the acoustic research on main rotor wake/tail rotor interactions concluded that the work, "confirmed the importance of the main rotor wake/tail rotor interaction on the noise generated by a helicopter during cruise flight". This importance was clearly recognised by industry through a drive to develop quiet tail rotors $[29,30]$.

Knowing that vortical interactions played an important role in the radiated noise, the U.K. Royal Aerospace Establishment (RAE) instrumented a Puma tail rotor with pressure transducers at both the leading edge and trailing edge to assess, via a method developed by them, the azimuthal blade loadings. Although the flight tests were successful in the main, only limited pressure data were collected [31]. This work was continued in the early 1990's [7] using an instrumented tail rotor on a Westland Lynx leading and trailing edges (Fig. 6). Using the developed measured-data analysis method [32], details of the tail rotor loading were readily obtained. In Fig. 7 single revolution variations of the leading edge pressure coefficients are presented. The resultant data, though informative, provide no information on the detailed chordal passage of the interacting vortices. Nonetheless, analysis of the data allowed the effect on the tail-rotor disc loading to be clearly highlighted. In Fig. 7 there is an obvious impulsive event where the leading edge pressure coefficient, at about $85 \%$ of rotor 
radius, experiences a sudden change from -1.0 to -2.72 around the $180^{\circ}$ azimuth position.

The underlying mechanisms for impulsively generated acoustic emissions from the tail rotor can be schematically illustrated as shown in Fig. 8. This figure depicts a two-dimensional vortex street, representative of successive main rotor vortices, interacting with a representative tail rotor with a blade-top-forward rotation. The emitted noise is shown ideally in the form of bursts. The spacing between them is the main rotor blade-passing frequency whilst that of the bursts is the tail rotor bladepassing frequency. The resultant noise is termed 'Burble'. A typical emitted noise spectrum for a helicopter in forward flight is given in Fig. 9 where the main and tail rotor tones have been highlighted. While it is obvious that the tail rotor produces significant contributions to the noise spectrum at specific frequencies, it has also been established that its contribution to the underlying broadband noise is not insignificant [33].

\subsection{Basic features of orthogonal blade vortex interaction}

The tip vortices trailed from main rotor blades can interact with the tail rotor in a variety of ways depending on the helicopter flight condition. In addition to the range of potential interaction geometries, the actual form that the interaction takes can vary depending on the relative timing of the main and tail rotor blade passes. In fact, the isolated convecting main rotor vortex can interact directly with a tail rotor blade or with the wake from the blade. In the latter case, the nature of the interaction also differs depending on whether the tail rotor blade wake is still in the roll-up process or whether it has reached the fully rolled up state. All of these interactions can influence the tail rotor performance and can occur at the same flight state during a relatively short time frame. This makes identification of the pure OBVI event from flight data problematic.

It is well known that a helicopter in forward flight can generate a flow field similar to that of a fixed wing aircraft (Fig. 10). These vortices are generated from the individual blade tip vortices rolling around each other to form larger trailed structures. This is somewhat different from the idealised wake structure presented in Fig.1. that does not model the roll-up of the outer wake segments into the large trailed structures. Both wake representations do, however, contain a series of connecting transverse tip vortex segments that are produced by the passage of the rotor blades around the forward and aft regions of the rotor disk. Thus, in forward flight the tail rotor will be in the centre of the main rotor wake and will cut each of these transverse vortex segments in sequence as they are trailed from the rear of the disc. The interactions of these segments with the tail rotor blades produce the OBVI events.

Over the past few decades, interest in the structure of rotor trailing vortices has developed as the need for greater fidelity in aerodynamic modelling has increased. Initially, numerical simulations were based on fixed wing data supplemented by rotor test data and flow visualisation. Latterly, however, difficult and detailed surveys of the rotor trailing vortices yielded additional and important information on the wake vortices including velocity components, vorticity distributions and spacial development $[34,35]$. The dominant features, however, of both the fixed and rotating 
wing tip vortices are their swirl and axial component velocities (Fig. 11). Both of these components have a role to play in determining the response to an OBVI event. The general features of the interaction, as manifest on helicopter tail rotors, are described below.

The blade response to an orthogonal interaction is characterised by impulsive and convective components [36]. During the early part of the interaction, when the vortex is initially cut, the axial velocity component of the interacting vortex is stopped abruptly. On the lower surface of the rotor blade, where the axial flow is towards the blade, this results in an impulsive compression and thickening of the vortex (Fig. 12). The opposite is true for the upper surface where there is an impulsive suction and a thinning of the vortex. This impulsive interaction is, however, short lived and is followed by a convective suction-wave corresponding to the passage of the vortex over the blade surfaces. The strengths of the lower and upper convective waves differ as a consequence of the thickening and thinning of the cut vortex. For constant vortex strength, the vortex thickening reduces the strength of the convective wave whilst thinning enhances the suction. Once the interaction is over and the interacting vortex leaves the trailing edge of the blade there is some evidence of re-combination of the upper and lower surface segments with the re-establishment of an axial flow component [37]. This may happen prior to the cut from the following blade but, given the timescales involved, the axial flow component may not fully recover.

This general description of OBVI to some extent understates the complexity of the process. Indeed, as a result of recent research, much more is understood about the phenomenon and progress has been made in modelling it. These developments are discussed in the following sections.

\section{Experimental studies of orthogonal blade vortex interaction}

One of the major problems associated with the study of OBVI is the need to isolate the phenomenon from the effects of the surrounding flow field. This is not straightforward because it requires a vortex with the same characteristics as that of a main rotor tip vortex to be produced in an otherwise aerodynamically clean environment. Most experimental model studies of tail rotor BVI have, therefore, not attempted this but instead have either been carried out as part of full aircraft configuration studies or have been acoustic studies of main and tail rotor interference. One of the earliest studies was that of White et al. [38] who conducted a wind tunnel based study of the acoustics of a model main and tail rotor configuration. This study highlighted that the acoustic emissions from the tail rotor were sensitive to a range of parameters including the direction of rotation of the tail rotor, the tail rotor tip speed and the spacing between the tail rotor and the tail fin. It was also established that the location of the interaction on the tail rotor disk was also an important factor. A similar study by Pegg and Shidler [39] in the NASA Langley anechoic facility, using a model that allowed the position of the tail rotor relative to the main rotor and the direction of the tail rotor to be varied, identified noise sources associated with the interaction of the tail rotor with both the main rotor tip vortex and its turbulent wake.

In model acoustic tests there is usually some concern that that measured results may not be representative of the full-scale. This was investigated by Tadghighi and 
Cheesman [40], who utilised an isolated $1.35 \mathrm{~m}$ diameter model tail rotor to obtain aeroacoustic measurements and compared these with full-scale results. They found that the results were both representative of the full-scale and consistent with theoretical analyses. They did, however, suggest that the turbulence level in the test facility could be an important factor depending on the test Reynolds number. Their conclusions on the applicability of model testing were later echoed by Sternfeld and Schaeffer [41] in a more generalised study of the use of models in helicopter noise research.

In addition to the reduced costs associated with model testing when compared to flight tests, there is the added advantage that the test environment is more controllable. Another advantage is the possibility of testing the tail rotor in isolation from the main rotor to provide a baseline noise signature against which interaction effects can be studied. This approach was adopted by Fitzgerald and Kohlhepp [42] in their tests of a $1 / 5^{\text {th }}$ scale main rotor, tail rotor and fuselage combination in the NASA Langley $14 \mathrm{ft} x 22 \mathrm{ft}$ wind tunnel. Interestingly, they found that the tail rotor noise was increased when it was operated in the absence of the main rotor and suggested that this indicated that the tail rotor inflow was improved when the main rotor was present. Similar results were presented by Martin et al. [33] from tests in the same facility. Additionally, however, they demonstrated that directivity strongly influences the relative dominance of the acoustic emissions from the main and tail rotor. Arguably the most comprehensive test of this type was on a $40 \%$ scaled model of the BO105 main rotor/tail rotor system in the DNW wind tunnel $[43,44]$. Both rotors were dynamically scaled and, in addition to the extensive acoustic measuring equipment used in the test, one of the tail rotor blades was instrumented with twenty miniature pressure transducers. Once again, it was shown that the noise from the tail rotor in isolation was greater than when it was operated in combination with the main rotor. The primary source of the impulsive noise from the tail rotor was identified as the interaction of the tail rotor blades with the tail rotor wake. Indeed, in combined operation, it was almost impossible to discern main rotor tip vortex interactions on the tail rotor because of these 'self' BVIs. There were, however, clear differences between the blade pressure time histories for isolated and combined operation that were attributed to interference effects between the two rotor wakes.

\subsection{Early experimental studies of orthogonal blade vortex interaction}

The studies described above serve to illustrate the problems of isolating specific OBVI events in the complex aerodynamic environment of a combined helicopter rotor system. Is clear, however, that the events do occur and that the noise emitted from the tail rotor is influenced by them. There is, therefore, a need to understand the nature of the interaction so that acoustic models can properly represent it in global rotor calculations.

One of the first attempts to isolate the phenomenon was made in a series of experiments conducted by Shlinker and Amiet [45] in support of their computational model development. In their experiments, an isolated vortex was generated from the tip of a semi-span wing. A model rotor was then placed in the path of the tip vortex such that the rotor disk was normal to the vortex axis (Fig. 13). In this experiment acoustic spectra, pressure signatures and sound directivity were measured but, 
although the interaction was observed to generate harmonic noise and impulsive waveforms, the detail of the interaction phenomenon remained obscured.

An almost identical approach was adopted in work carried out in the Bolt Beranek and Newman high-speed acoustic wind tunnel by Ahmadi [46] and later Cary [47]. Ahmadi used a tail rotor blade instrumented with four pressure transducers, located at $10 \%$ chord in upper and lower surface pairs at two span locations, to establish that the BVI events produced impulsive pressures near the leading edge. In the follow-on study by Cary, the interactions were photographed using high-speed stroboscopic illumination and he was able to determine that the incident vortex diffused rapidly on both sides of the rotor blade. He also observed that the influence of the tail rotor wake on the incident vortex was destabilizing.

Although these experiments provided further insight into the OBVI phenomenon, the relatively small number of surface pressure transducers and limited capability of the photographic system restricted the detailed information that could be obtained. Additionally, aspects of the set-up of the experiments were unrepresentative of helicopter flows. The fact that the free stream flow was in the same direction as the rotor slipstream meant that the experiment was more akin to a propeller type scenario than a tail rotor. This, with hindsight, may have been an advantage because 'self' BVI events would be avoided. More importantly, however, the test geometry caused the vortex to be cut repeatedly along its length by the blades of the rotor. This is unlike a helicopter scenario where repeated cutting of a main rotor vortex by tail rotor blades is likely to occur at approximately the same geometric location on the tip vortex helix as the vortex passes through the tail rotor. This difference may have implications in terms of the potential re-establishment of the vortical structure in the post-interaction stage and may also reduce the magnitude of the axial flow in the vortex core.

Possibly a more representative study, although not of pure OBVI, was conducted by Johnston and Sullivan [48] in an experiment that was actually designed to examine unsteady wing pressures in the wake of a propeller. In this experiment, a high aspect ratio wing instrumented with small pressure transducers was placed one chord length behind a propeller in a low speed wind tunnel. By having sixteen chordwise pressure ports at the mid span of the wing, it was possible to record the passage of vortical structures across the wing. Also, marking the propeller tip vortex with smoke allowed the interaction to be visualized. The visualisation study showed that the vortex filament thickened on one side of the interacting wing and thinned on the other side. This was attributed to the axial flow in the vortex core.

Following these studies, many aspects of the OBVI phenomenon still remained unclear. Two major studies, conducted at the University of Iowa in the US and the University of Glasgow in the UK, have gone a considerable way towards addressing this knowledge gap. These studies, which have respectively focussed on the fluid dynamics of the interaction and the blade response to the interaction, and have provided considerably more detail on both aspects of the interaction. The main findings of these two studies are presented in the following sections. 
The studies conducted at the University of Iowa examined the vortex response to OBVI for a towed blade passing orthogonally through an intake vortex, over a range of different values of the blade towing speed. The governing dimensionless parameters for OBVI in this simple configuration include the impact parameter $I=2 \pi a U / \Gamma$, the axial flow parameter $A=2 \pi a w_{0} / \Gamma$, the blade thickness parameter $T=D / a$, and the vortex Reynolds number $\operatorname{Re}_{V}=\Gamma / v$. Here $a$ and $\Gamma$ denote the vortex equilibrium core radius and circulation, $w_{0}$ is the maximum value of the vortex axial velocity, $D$ is the blade thickness, and $v$ is the kinematic viscosity (Fig. 14 ). For cases with $A$ greater than a critical value (equal to approximately 0.71 for a Rankine vortex), the vortex is said to be supercritical and linear axisymmetric waves on the vortex core are swept downstream. For values of $A$ less than this critical value, the vortex is subcritical and linear axisymmetric waves can propagate in either direction on the vortex core.

The series of experiments conducted at Iowa by Krishnamoorthy and Marshall $[8,49,50]$ particularly examine the effect of the impact parameter on OBVI for a subcritical vortex. The experiments were performed by forming an intake vortex in a cylindrical tank, with tangential jets at the tank top and an orifice through which liquid was removed at the bottom (Fig. 15). The cylindrical tank was contained within an outer rectangular tank, both of which were filled with water. An inverted funnel was placed at the top of the tank in order to eliminate vortex wandering. A slot at about the mid-section of the cylindrical tank allowed a blade to be towed through, penetrating into and cutting through the vortex core.

Laser-induced fluorescence (LIF) was used to separately visualize fluid arising from the vortex core and that arising from the blade boundary layer, with dye that fluoresces red injected into the top of the vortex core (and carried downward by the vortex axial flow) and dye that fluoresces yellow injected into a series of small holes along the blade leading edge. An argon-ion laser was used for planar visualization, in both the vertical and horizontal planes, and a Nd:YAG laser was used for volumetric visualization. Particle-image velocimetry (PIV) was used to measure the ambient velocity within the intake vortex. The axial flow parameter, vortex Reynolds number, and blade thickness parameter for all experiments were held constant at $A=0.19 \pm 0.06, \operatorname{Re}_{V}=2.5 \times 10^{4}$, and $T=1.2$. The impact parameter was varied over the interval $I=0.02-0.4$.

These experiments focused on the effect of the blade cutting on the vortex core response, the effect of the vortex on the blade boundary layer, and the interaction of the shed boundary layer with the incident vortex. Qualitatively different types of interaction were observed for cases with relatively large or small impact parameter. For large impact parameter (which might be termed a weak-vortex interaction), the blade moves toward the vortex core and penetrates into the core without separation of the blade boundary layer. For small impact parameter (termed a strong-vortex interaction), vorticity from the blade boundary layer is ejected from the blade leading edge and pulled backward by the vortex-induced velocity, eventually wrapping around and becoming entrained into the vortex core. 


\subsubsection{Weak-vortex regime}

A planar LIF photograph showing the core response of a vortex with downward axial flow to cutting by a blade at large impact parameter is given in Fig. 16 (from Marshall and Krishnamoorthy, [50]). The dye injected into the vortex core is cut off prior to cutting, so that the dye marks only the lateral boundary of the vortex core. Fluid from the blade boundary layer has been entrained into the vortex core on both sides of the blade. The core of the columnar vortex is observed to increase on the upper part of the vortex, where the vortex lines are compressed by the axial flow, and to decrease on the lower part of the vortex, where the vortex lines are stretched by the axial flow.

The observed variation in core radius in Fig. 16 is consistent with the prediction of the plug-flow model of Marshall [51]. This model assumes that the vortex has uniform axial vorticity and axial velocity over a cross-section to derive a set of onedimensional hyperbolic equations, analogous to the gas-dynamic equations, for the variation of vortex core radius $\sigma(z, t)$ and axial velocity $w(z, t)$ of the form

$$
2 \frac{D \sigma}{D t}+\sigma \frac{\partial w}{\partial z}=0, \quad \frac{\sigma^{2}}{2} \frac{D w}{D t}=-\frac{\Gamma^{2}}{8 \pi^{2} \sigma} \frac{\partial \sigma}{\partial z}
$$

as a function of time and axial distance ( $z$ ) along the vortex core (see also [52]). Here the material derivative is defined in terms of the axial convection only, as

$$
\frac{D f}{D t} \equiv \frac{\partial f}{\partial t}+w \frac{\partial f}{\partial z}
$$

These equations admit a solution from the method of characteristics of the form

$$
\begin{aligned}
& J^{+}=w-2 c=\text { const } \text { on } \frac{d z}{d t}=w+c \quad\left(C^{+} \text {characteristics }\right) \\
& J^{-}=w+2 c=\text { const } \text { on } \frac{d z}{d t}=w-c \quad\left(C^{-} \text {characteristics }\right)
\end{aligned}
$$

where the speed $c$ of long axisymmetric waves is given by

$$
c=\frac{\Gamma}{\sqrt{8} \pi \sigma}
$$

This model has an analytical solution for the problem of impulsive cutting of a subcritical columnar vortex, which is mathematically analogous to the problem of impulsive piston motion in a shock tube, or to the dam-break problem in shallowwater hydraulics. For a subcritical vortex, the plug-flow model predicts that a "vortex shock" will propagate upstream on the vortex, leading to an increase in core radius in 
the upper section of the vortex behind the shock to a value $\sigma_{C}$. [Here the terms "shock" and "expansion wave" are used in analogy to gas dynamics, but it is the vortex core radius that varies along the vortex, rather than the fluid density.] The vortex shock speed is given by

$$
W=\frac{\Gamma}{2 \pi \sigma_{C}}\left[\frac{\ln \left(\sigma_{C} / a\right)}{\sigma_{C}^{2} / a^{2}-1}\right]^{1 / 2}
$$

and the core radius near the blade is obtained by solution of the non-linear equation

$$
\sigma_{C} / a=\frac{1}{A}\left[\left(\sigma_{C}^{2} / a^{2}-1\right) \ln \left(\sigma_{C} / a\right)\right]^{1 / 2} \geq 1
$$

On the lower side of the blade, where the vortex lines are stretched by the axial flow, a vortex "expansion wave" propagates downstream, such that the core radius changes linearly with axial distance between the rear and the front locations of the expansion wave, given by

$$
z_{F}=\left(w_{0}-c_{0}\right) t, \quad z_{R}=-c_{E} t
$$

where $c_{E}=c_{0}-w_{0} / 2$ is the value of $c$ behind the expansion wave and $c_{0}$ and $w_{0}$ are the ambient values of $c$ and $w$ on the vortex. The core radius on the lower side of the vortex behind the expansion wave is obtained from the model solution as

$$
\sigma_{E} / a=1 /\left[1+\frac{\sqrt{2}}{2} A\right] \leq 1
$$

One consequence of vortex cutting observed in the experiments is development of a vortex breakdown that propagates upstream on the vortex core. The breakdown has a bubble-type form, which is usually followed by a double-helix breakdown, as shown in Fig. 17. The propagation speed of the vortex breakdown is found to agree well with the predicted vortex shock speed from the plug-flow model [49]. Similar bulging waves on the vortex core were observed to form in the experiments of vortex ring cutting by a thin plate by Weigand and Gharib [53].

\subsubsection{Strong-vortex regime}

As the vortex-induced velocity becomes sufficiently strong compared to the impact velocity $U$, corresponding to small impact parameter values, the boundary layer separates from the blade leading edge prior to the impact of the blade on the vortex core. A planar LIF photograph showing the boundary layer ejection, taken in the horizontal blade centerplane, is given in Fig. 18. The boundary-layer vorticity is first ejected at an angle of approximately $45^{\circ}$ from the line connecting the vortex center to the closest point on the blade leading edge. In a related study by Marshall [54] on orthogonal vortex-cylinder interaction, it was shown that onset of boundary layer ejection occurs when the gradient of the vortex-induced velocity component $u^{\prime}$ in the direction of blade motion $U$ along the blade normal at the leading edge exceeds the normal gradient $4 U / D$ due to the free-stream velocity relative to the blade. When 
this condition is satisfied, the inviscid flow at a position close to the blade leading edge, but located slightly off the boundary, will be oriented away from the blade (in a coordinate system attached to the blade), such as to advect boundary-layer fluid away from the blade leading edge. Marshall [54] demonstrated that this criterion results in a simple formula, for the critical distance $S_{\text {crit }}$ between the vortex center and the blade leading edge at which boundary layer is ejected, of the form

$$
S_{c r i t} U / \Gamma=0.055
$$

The prediction from (9), plotted in Fig. 19, is in excellent agreement with experimental data for boundary-layer ejection. While this result is developed for vortex-cylinder interaction, a similar argument would be expected to hold for the OBVI problem since the onset of boundary layer separation depends principally on the flow in the region of the blade leading edge.

The ejected boundary layer fluid forms a series of vortex loops, each connected at two points to the blade boundary layer, which wrap around the vortex core. The structure of these loops is shown using a volumetric LIF image in Fig. 20a. As the ejected vortex loops wrap around the columnar vortex, they are gradually entrained into the vortex core due to their self-induced velocity. A computational study of the response of a columnar vortex to a wrapped vortex loop is given by Krishnamoorthy and Marshall [8]. This study examined the process by which azimuthal vorticity is generated within the columnar vortex through a combination of radial tilting of the vortex lines by the flow induced by the vortex loop, followed by azimuthal tilting of the vortex lines due to the radial variation of the azimuthal velocity of the columnar

vortex. This process results in the generation of azimuthal vorticity streaks within the columnar vortex that have azimuthal vorticity of an opposite sign to that within the nearby vortex loop leg (see also Marshall et al. [55]).

Over sufficient time, the ejected vorticity forms a sheath of turbulence that wraps locally around the outer surface of the columnar vortex, as shown in Fig. 20b. The turbulence external to the vortex, formed from vorticity shed from the blade, gradually eats into the outer part of the columnar vortex core, and over time can have a significant impact on the vortex axial flow. This principle was demonstrated by Sun and Marshall [56] in an experiment in which a sphere was towed to within a specified position outside of the vortex core and then stopped. The subsequent interaction of the sphere wake with the columnar vortex was then examined. It was found that the presence of the sphere wake could lead to both significant variation in core area of the vortex (as is also evident in Fig. 20b) and to formation of an upward-propagating breakdown on the columnar vortex.

\subsection{Experimental studies at the University of Glasgow}

Unlike the studies at Iowa, the work at the University of Glasgow has focused on examining the blade response to OBVI in a series of wind tunnel based studies. These studies have attempted to replicate the basic geometry of a tail rotor blade vortex interaction while, at the same time, isolating the interaction from the complex flow field of a full main rotor/tail rotor configuration. Preliminary work was carried out by Copland et al. [57] to determine an appropriate means of generating the interaction 
and to demonstrate the feasibility of the methodology. The basic set-up developed at this stage was subsequently used to examine several aspects of orthogonal interaction via unsteady pressure measurement and particle image velocimetry (PIV) studies.

The most important aspect of experimentally simulating any helicopter blade vortex interactions is ensuring that the vortex generated during the experiment is representative of a rotor tip vortex. The most obvious way to satisfy this condition is to use a rotor as the vortex generator in the experiment. Depending on operating conditions, however, the wake produced by a multi-bladed rotor is generally laced not only with the primary tip vortices but also with secondary vortices resulting from the interaction of the primary vortices with the rotating blades. Isolating the response due to a specific interaction in this type of flow field is extremely difficult. One means of minimising this problem is to reduce the number of blades in the rotor system, if possible, to one. The rotor wake can be idealised further if the rotor blade only produces a tip vortex for part of its cycle. In essence, this was the approach to vortex generation adopted in the work conducted at the University of Glasgow [58]. The interacting vortex was produced by a single bladed rotor located upstream of the wind tunnel working section and the azimuthal extent of the rotor wake was controlled by varying the blade incidence around the cycle.

The fact that the vortex was to be produced by a rotating blade placed in either the contraction or settling chamber of a low speed wind tunnel meant that the generated vortex system would be subject to the accelerating flow field associated with the contraction of the wind tunnel. A numerical model of the flow field was, therefore, used to calculate the evolution of the wake geometry for a range of rotor axis positions, blade geometries and operating conditions of the rotor and the wind tunnel. From this work, it was possible to determine an appropriate test configuration that would produce an orthogonal interaction with either a rotating or stationary blade in the wind tunnel working section.

The features of the experimental facility that had to be modelled were the vortical wake structure generated from the rotating blade and the main flow through the wind tunnel. The rotor wake was represented by a free wake vortex model consisting of a lattice of shed and trailed vortex elements which were generated using classical lifting line theory. This vortex system was then convected through the contraction and working section of the wind tunnel with the superposition of the local velocity, calculated via a three dimensional source panel method, and the induced velocity components from the vortex elements. Due to the inviscid nature of this model, no account was taken of vortex dissipation or the change in vortex strength due to deformation of the vortex elements, and so the strength of each vortex element was invariant with time.

A source panel method was used to represent the constraining effects of the wind tunnel walls. The method adopted was based on the work of Hess and Smith [59] and was chosen for its relative simplicity and adaptability to internal flows. Due to the simple geometry of the modelled portion of the wind tunnel and the non-lifting nature of the body, it was deemed sufficient to represent the wall surfaces using plane quadrilateral elements with constant source distributions. 
The internal surface of the wind tunnel was discretised into approximately 1000 individual quadrilateral elements representing the settling chamber, contraction, working section and diffuser (Fig. 21). This representation gave sufficient distance upstream of the vortex generator location to be out of its disturbance environment, and extended far enough downstream to allow the vortex system to convect through and out of the working section. Since the accuracy of such calculations can depend not only on the number of quadrilateral elements used but also on the manner in which these elements are distributed over the surface, a non-uniform distribution of panels was used. In particular, panels were concentrated in the region of the contraction (area of high curvature) and the working section (area of interest).

The panel method and the wake model were loosely coupled in that the induced velocities from the wake were not taken into account when calculating the source strengths of the panels. This was done for efficiency since strongly linking the two models, by calculating the induced velocities from the wake at each panel collocation point, would have significantly increased the computational time and so limited the range of cases which could have been considered in the parametric study. This approach was considered acceptable due to the low blockage presented by the generator assembly.

At a later stage, hot-wire measurements were used to verify the free-wake model results for the actual test configuration [60]. This was done by taking a series of simultaneous measurements at two positions in the wind tunnel, one of which was fixed and the other varied. In each case, the vertical heights of the probes were adjusted until the maximum response due to the vortex passage was obtained. The measured time difference between the vortex passage at the two points then provided a direct measurement of relative wake position. These measurements were compared with a two-dimensional projection of the computed wake structure at an equivalent time, which is shown in Fig. 22. Very good agreement is observed, suggesting that the coupled panel method and free wake model provided an acceptable representation of the wake geometry in the wind tunnel.

The first test facility was designed for the Glasgow University $1.15 \mathrm{~m} \times 0.85 \mathrm{~m}$ low speed wind tunnel. The vortex generator used in this tunnel was essentially a rotor of radius $0.75 \mathrm{~m}$ that had a single rectangular planform blade of chord $0.1 \mathrm{~m}$ with a NACA 0015 cross section. During rotation, the blade pitch was varied using a springloaded pitch link running on a cylindrical cam configured such that the blade pitch varied in four equivalent $\left(90^{\circ}\right)$ phases of azimuth. The first phase set the blade at zero incidence while the blade was pointing into the settling chamber $\left(45^{\circ}\right.$ azimuthal travel on either side of the wind tunnel centre line). In the next two phases of motion, the blade was pitched from zero to $10^{\circ}$, before traversing the working section at a constant $10^{\circ}$ incidence. In the final $90^{\circ}$ phase, the spring loaded pitch link forced the blade to overcome its aerodynamic and inertial loads and follow the cam as it returned to zero degrees.

The vortex generator produced a curved, three-dimensional vortex that convected through the wind tunnel working section. A stationary NACA 0015 blade of chord $152.4 \mathrm{~mm}$ and overall span $944 \mathrm{~mm}$ was placed in the path of the convecting vortex in order to study the interaction of the vortex with the blade. The experimental setup allowed for variation of the geometric incidence of the blade and of its lateral position 
in the wind tunnel. The blade was instrumented with a chordal array of 30 miniature pressure transducers mounted around the surface of the blade. For all tests in this facility the freestream velocity was fixed at $20 \mathrm{~ms}^{-1}$ and the rotational speed of the vortex generator was 500 RPM. Based on these conditions, the nominal interacting blade Reynolds number was $2 \times 10^{5}$. The test set-up is shown schematically in Fig. 23. Tests were conducted with the blade in two lateral positions, each $225 \mathrm{~mm}$ on either side of the tunnel centreline. In one of these positions, the interaction had been predicted to be orthogonal whereas in the other position the vortex encountered the blade at an angle of 42 degrees [61].

A second test facility was also developed in the Glasgow University $2.65 \mathrm{~m} \times 2.04 \mathrm{~m}$ 'Argyll' low speed wind tunnel [62]. This facility was essentially a scaled-up version of that used in the smaller tunnel but the vortex generator design was refined to include a special hard-chromed cam-plate together with a fully articulated rotor hub, which incorporated flap and lag hinges with a cantilevered flap spring and elastomeric lag dampers. It had a rotor of radius $1.6 \mathrm{~m}$ with a single rectangular planform blade of chord $0.16 \mathrm{~m}$ with a NACA 0015 cross section.

Two series of interaction experiments were conducted in this facility. In the first, a NACA 0015 blade of chord $275 \mathrm{~mm}$ was mounted vertically in the path of the vortex, Fig. 24, at a location $4 \mathrm{~m}$, or 14.55 blade chord lengths, downstream of the rotor rig axis [63]. The blade was placed on the right side $370 \mathrm{~mm}$ from the tunnel center-line, facing into the settling chamber. At this position, the vortex was predicted to collide nominally orthogonally with the blade which was instrumented with the three chordal arrays of 27 miniature pressure transducers. The separation distance between each array was $68.75 \mathrm{~mm}$. Additional transducers were placed between the chordal arrays to provide detail of spanwise loading distributions at six chordal locations. During testing the blade could be set at different incidence values and it could be moved vertically to allow the relative location of the chordal arrays with respect to the vortex core to be changed.

In the second series of tests, a model tail rotor was placed in the path of the convecting vortex in order to study the vortex interaction with a rotating blade [64]. The tail rotor system, which had been designed at the University of Southampton, was supplied by Westland Helicopters Limited, but the blades were re-designed and constructed by the University of Glasgow in order to accommodate appropriate pressure transducers. The rotor had two constant-chord blades of the NACA0015 aerofoil section. The radius of the rotor was $428 \mathrm{~mm}$ and the chord of the blades was $100 \mathrm{~mm}$. One of the blades was instrumented with nine miniature pressure transducers mounted on the upper surface and six on the lower surface (Fig. 25). The transducers were arranged in three chordal arrays, the distances of which from the rotational centre were $69 \%, 75 \%$ and $81 \%$ of the rotor radius. Each chordal array had three transducers on the upper surface, positioned at $6 \%, 30 \%$ and $75 \%$ of the chord, and two on the lower surface, located at $10 \%$ and $65 \%$ of the chord.

The experimental set-up allowed the tail rotor to be mounted at three different vertical positions in the wind tunnel working section. The tail rotor was installed such that the axial core flow of the vortex was toward the rotor disc from the "clean" side, thus keeping the disturbance of the rotor driving and support systems on the vortex minimal prior to the vortex collision. The rotor centre was about $4 \mathrm{~m}$ downstream of 
the vortex generator rotor axis. Like the stationary blade, the tail rotor disk was located on the right side $370 \mathrm{~mm}$ from the tunnel centre-line, facing into the settling chamber. Figure 26 illustrates the experimental set up in the wind tunnel as viewed from above and includes details such as the direction of axial core flow in the interacting vortex and the definition of positive and negative tail rotor pitch angle. The trailing vortex shape shown in Fig. 26 is indicative of that predicted by the freewake simulation.

Pressure data for each transducer were recorded using a BE256 data logger in a single 32000 sample block at $20 \mathrm{kHz}$ sampling rate. This sample rate and size allowed approximately 13 rotor revolutions of data capture. Five blocks of data were obtained at each blade incidence.

In all of the test programmes hot wire velocity measurements were taken at the position of the interacting blade, or rotor, position prior to the installation of the blade. By traversing the probe vertically through the working section at this location, the transient vortex velocity field was acquired. The height of vortex core passage was considered to be the position where, on average, the maximum vertical velocity signal (v) was recorded (it is directly related to the vortex tangential velocity, through a simple transformation [60]). The interacting blade, or rotor, was then installed with the transducers placed at the desired height in relation to the vortex core path. A typical hot-wire vertical velocity record corresponding to the vortex core passage height is shown in Fig. 27. Some variations between successive vortices were observed due to vortex wandering and local turbulence levels in both facilities.

Independent velocity measurements were also made in the smaller wind tunnel using a particle image velocimetry (PIV) system based on a Spectra-Physics Nd:Yag laser. By operating the laser in a double-pulsed mode, two images of the flow field could be obtained with very small time separation $(50 \mu \mathrm{s})$. A standard cross-correlation and sub-pixel peak detection routine was used to determine the mean velocity at various locations in the region of interest. In this work, two PIV systems were used to obtain separate flow field results of the same vortex but at different locations along the chord. The system uses two lasers and two cameras carefully timed to give results separated in time. The flow was seeded with $1-2 \mu \mathrm{m}$ oil smoke particles distributed throughout the flow. The laser light was passed through an optical arrangement which converted the beam into a laser sheet which was passed through the working section and illuminated the flow seeding. The particle displacements could then be recorded using two Kodak Megaplus ES 1.0 cameras of $1 \mathrm{k}$ x $1 \mathrm{k}$ resolution. The experiment was designed so that each camera detected the flow pattern illuminated by its own laser. Full details of the PIV technique can be found in Green et al. [65].

Particle image velocimetry (PIV) was also used to verify the position of the vortex and to quantify the total circulation about the core. A relatively high rotational velocity gradient exists within the vortex core that subjects entrained smoke particles to high centripetal and coriolis accelerations. Any particles initially within the core follow a spiral path which ejects them into the surrounding fluid. This was noticed as a dramatic drop in seed particle density within the vortex core region and hence the PIV system has poor resolution of the velocities within the core. The PIV measurements are therefore more useful for measuring the total circulation of the vortex rather than information within the core. Errors induced in the circulation 
measurements by vortex wandering in probe-based techniques can be reduced using PIV if the sampling region is larger than the wandering amplitude of the vortex. A summary of the vortex parameters for the nominal test conditions in the smaller wind tunnel, measured using both PIV and Hot-wire anemometry, can be found in Table 1. The hot wire results were used to obtain estimates of the vortex core size. This is defined as the distance between the peak tangential velocities on a line passing through the vortex centre. The PIV measurements were used to obtain the vortex circulation. The hot wire measurements were also used to obtain circulation measurements and the differences are indicated on the table. The benefits of using the PIV system are obvious with the uncertainty in the measurements reduced by $50 \%$.

The vortex information presented in Table 1 was used to estimate the blade impact parameter which, for this case was 2.16. This value implies that a weak interaction occurs during the experiments with no boundary layer separation before the vortex collides with the leading edge. This is also the dominant tail rotor interaction mechanism for a helicopter in forward flight with an advance ratio greater than about 0.1 . The corresponding values for the test programme in the larger wind tunnel were also indicative of a weak interaction.

The last row of Table 1 is indicative of a significant cross-stream velocity associated with the vortex core axial flow. As discussed previously, the value of the axial flow parameter, $A=0.89$, determines some aspects of the vortex dynamics during a vortex cut. For these experiments this value indicates that the vortex is supercritical and only downstream area-waves of small amplitude can be supported on the vortex core [66]. If the vortex is supercritical it can be expected that when the vortex impacts with the instrumented blade, a jet-like flow will occur on the lower surface and a thinning of the vortex core will occur on the upper surface. In the experiments in the larger wind tunnel, both sub and super-critical cases were considered and this is discussed further in the following section.

In the following sections results are presented for a range of interaction cases. Unless otherwise stated, the results were obtained in the smaller wind tunnel facility.

\subsubsection{Orthogonal interaction on a zero-loaded blade}

For the basic orthogonal interaction tests studied in the smaller wind tunnel, the interacting blade was placed $225 \mathrm{~mm}$ to the right of the wind tunnel working section centreline. It was known, from the results of the numerical model and the hot-wire measurements, that the vortex approached this location at $90^{\circ}$ to the mean flow direction. The unsteady blade pressures were converted to pressure coefficients using the recorded freestream velocity, air density and pressure. When interpreting the results presented below, it should also be noted here that the upper surface experiences a vortex core flow away from the surface while the lower surface experiences a core flow directed toward the surface.

Figures 28 illustrates the temporal variation of the pressure data recorded as the vortex was cut by the blade when the transducers were placed in the path of the core. In each plot, the steady component of the pressure signal has been removed in order to increase the clarity of the results. The steady component was determined by averaging 32000 pressure samples obtained while the rotor rig was idle and making sure the interacting blade was not in the rotor blade wake. Hence, -(Cpu-Cps) is plotted on the 
vertical axis with the chord position of the transducers $(x / c)$ on one axis and nondimensional time $(t V / c)$ on the axis in the foreground. To further increase the clarity of the plots only every fifth data sample is presented. The upper surface interaction data in Fig. 28a shows a strong suction peak at the leading edge when the vortex first encounters the blade. As the vortex passes over the surface, the suction peak diminishes, but is still significant over the remainder of the chord. For the lower surface (Fig. 28b), an increase in pressure occurs just downstream of the leading edge but is rapidly diminished as the vortex travels over the blade surface. At approximately the quarter chord point, the pressure ridge transforms into a slight suction ridge that continues to convect towards the trailing edge.

The surface pressure results obtained are interesting and illustrate the complexity of the vortex interaction. Although the only results presented here have been for the transducer array aligned with the vortex core, measurements were made, and have been fully documented [61], above and below the vortex core path. Common to all records is the presence of a suction peak on the upper and a pressure peak on the lower surface in the vicinity of the leading edge. This aerodynamic response can be explained in terms of the core axial flow. For the current configuration, the vortex axial flow is expected to travel towards the lower surface and away from the upper surface hence initiating the pressure and suction peaks observed. After the initial vortex cut, any axial core flow will be greatly reduced and the low pressure within the vortex core would be allowed to dominate, thereby creating a suction ridge over the surface.

Basic orthogonal interaction data obtained in the larger wind tunnel facility are presented in Figs. 29a \&b. For this case, the wind tunnel velocity was set at $50 \mathrm{~m} / \mathrm{s}$ and the measurements were, once again, taken at the vortex core passage height. These results exhibit a generally lower level of response in terms of the main unsteady features. This may be attributed to the higher value of the impact parameter in this case, 36.84, producing a generally weaker interaction. It is also apparent that the unsteady response to the interaction not as rapid, short-lived and localized as in the smaller tunnel whereas the duration of the interaction is much longer. One likely reason for this is the much larger ratio of the vortex core radius to airfoil chord in this case $(0.328)$ compared with the smaller tunnel $(0.065)$.

The response of the vortex to the interaction in the smaller wind tunnel was investigated using the PIV system. The rotor and laser system were synchronised so that the laser pulses illuminated the vortex as it passed through the required location(s) in the wind tunnel. By varying the delay between the two lasers and by moving the blade along the tunnel working section, the vortex interaction was investigated along the entire blade chord. The results presented here were obtained by placing the laser sheet $15 \mathrm{~mm}$ from the blade centre-line, parallel to the blade chord. At the maximum blade thickness the laser sheet was at a distance of only $3 \mathrm{~mm}$ from the lower blade surface.

Figures 30 show a series of vortex flow fields measured near the lower surface of the blade as the vortex collides with the blade. In each frame, the leading edge is indicated and the mean horizontal velocity component has been removed in order to highlight the vortex flow. In Fig. 30a the vortex is just upstream of the leading edge. Figure $30 \mathrm{~b}$ shows the same vortex $1.7 \mathrm{~ms}$ later, after it has interacted with the leading 
edge. Fig. 30b shows the velocity vectors spiralling outwards from the vortex core. This effect can be seen more clearly in Fig. 30c which shows the vortex at a location further downstream.

PIV measurements of the flow over the upper surface of the blade during the interaction were also performed by Early et al. [67]. Here the vortex core axial flow is directed away from the blade surface. As the vortex passed over the blade surface the PIV showed the flow spiralling inwards towards the vortex centre, suggesting a sinklike inflow. However the effective sink strength was much smaller in magnitude than the source strength for the lower surface interaction. The vortex core was observed to shrink only slightly in size and the vorticity levels generally remained constant along the chord. The differences in the flow behaviour between the two sides of the blade would appear to be consistent with the sense of the vortex core axial flow. For the lower surface the production of the source-like outflow after blade-vortex interaction originates from the blocking of the vortex core axial flow, but for the upper surface the vortex axial flow must be re-established to the non-interaction value a short distance away from the blade surface. This would tend to stretch the vortex and cause an in-flow close to the blade surface. These flow features are in general agreement with the findings of the work at Iowa.

The pressure data were integrated over the blade surface to give normal force and quarter chord pitching moment coefficients during the vortex interaction. Figure 31 plots the force and moment coefficient data against non-dimensionalised time for the transducer array aligned with the vortex core. A significant, impulsive normal force is experienced by the blade (Fig. 31a) as the vortex is cut by the leading edge. The normal force then decays as the vortex travels down the chord. The quarter chord pitching moment data (Fig. 31b) illustrates a large nose up moment is experienced by the blade as the vortex approaches the leading edge. A rapid change in pitching moment sign is subsequently observed which can be attributed to vortex passing the quarter chord point. Finally, pre-interaction pitching moment values are recovered after the vortex passes over the trailing edge.

The trends in normal force and pitching moment observed in the larger wind tunnel facility are similar in form to those presented above. For example, Fig. 32 presents the normal force coefficient variation corresponding to the pressure measurements shown in Figs 29. In addition, this figure also shows how the coefficients change for measurement positions above and below the vortex core centre. At the vortex core height, the magnitude of the $\mathrm{Cn}$ response is greatly reduced in comparison to the results from the smaller facility. This is a direct consequence of the greatly reduced axial flow velocity in relation to the free stream, $w_{o} / \mathrm{V}=0.044$, in the larger wind tunnel. Away from the vortex centreline, the normal force response changes depending on whether the measurement station is above or below the centre. It is possible that the rotation of the vortex plays a role in this behaviour but this is the subject of ongoing investigation.

\subsubsection{Effect of vortex approach angle}

The effect of a significant change in vortex approach angle was studied by placing the interacting blade $225 \mathrm{~mm}$ to the left of the wind tunnel centreline. At this location, both the hot-wire measurements and the numerical model indicated that the vortex approach angle was approximately $42^{\circ}$. Interestingly, as shown in Fig. 33, the general 
characteristics of the pressure response for this case were similar to those of the purely orthogonal case although the magnitude of the response was not as great. This is consistent with the general findings of Johnston and Sullivan in their propeller wake interaction experiments where the interactions were non-orthogonal. Some specific differences were, however, observed. In particular, the suction ridge over the trailing edge on the upper surface was not as sharp and there was no establishment of a suction ridge on the lower surface. Nevertheless, the marked similarity between this case and the purely orthogonal case suggests that the gross interaction characteristics are little altered by quite significant changes in vortex onset angle.

\subsubsection{Orthogonal interaction on a loaded blade}

The general form of the pressure response during interaction with a loaded blade is very similar in form to the zero incidence case. The main difference, at moderate incidence, is the magnitude of the suction and pressure peaks achieved. The measurements show that the suction peak on the upper surface leading edge is seen to intensify when the blade is set at positive incidence and reduce somewhat at negative incidence. This effect can be observed in more detail in Fig. 34a where peak suction measurements obtained during the interaction from a transducer placed at $\mathrm{x} / \mathrm{c}=0.009$ are plotted against blade incidence. Each data point represents an average of twenty interactions and the error bars indicate one standard deviation either side of the mean. Despite a significant scatter, thought to be due to vortex wandering, the results show the suction peak increases in magnitude on the upper surface as the blade incidence is increased. Further, a clear maximum is observed at $\alpha=6^{\circ}$. This maximum in suction peak also occurs at the same incidence setting for transducer locations further downstream. With the blade incidence set below $\alpha=-4^{\circ}$, the mean value in suction peak is reduced (compared with $\alpha=0^{\circ}$ ) and remains relatively constant.

On the lower surface, the change in incidence has a similar effect on the observed pressure pulse. As the incidence is increased in the positive sense the pressure pulse is reduced. When the incidence becomes negative the pressure pulse at the leading edge increases in magnitude. In Fig. 34b, the peak pressure results from twenty orthogonal vortex interactions have been extracted from a transducer on the lower surface and near the leading edge $(\mathrm{x} / \mathrm{c}=0.011)$. An increase in pressure pulse magnitude (denoted by a decrease in (Cpu-Cps)) with decreasing incidence can be observed from the results. The general form of the curve is monotonic except at negative incidence values beyond $\alpha=-5^{\circ}$. It is interesting to note that, in this respect, this behaviour is almost a mirror image of that presented in Fig. 34a.

The way in which the above changes are manifest in the normal force response is particularly interesting. Figure 35 shows the variation in the difference between the peak and time averaged normal force coefficients during the interaction over the range of incidence settings used in the experiments. The plotted value $\Delta \mathrm{Cn}$ therefore represents the impulsive normal load imparted to the blade by the interaction of the vortex only with the offset of the steady aerodynamics removed. Each data point in Fig. 35 represents the mean of twenty vortex interactions and the error-bars indicate the standard deviation. The results show that as the blade incidence is changed, the impulsive peak normal force experienced by the blade is approximately the same. Interestingly, force measurements taken during a simulated main rotor blade vortex interaction [68] produced a similar result despite the fact that the vortex interaction geometry was of a different type. 
It would appear that the peak magnitude in normal force is maintained due to the amplification and attenuation of suction and pressure peaks around the leading edge at different incidence settings. At positive incidence, the suction peak is increased while the pressure pulse is reduced and the opposite is true at negative incidence. Therefore, even if the upper and lower surface pressure distributions are altered, the overall effect on transient normal force is the same.

\subsubsection{The effect of vortex pre-cut on the interaction response}

In forward flight, the main rotor tip vortex may be cut several times by tail rotor blades after the initial interaction. The extent to which these secondary interactions are significant in terms of noise generation is presently unknown. In an attempt to gain a basic insight into this phenomenon, a series of experiments were conducted in which the vortex was pre-cut by a blade located one chord length upstream of the instrumented blade.

Surface pressure measurements of the vortex interaction when an upstream or preceding blade is installed are shown in Fig. 36. The results are of generally the same nature as the clean interaction tests but are reduced in magnitude. A suction ridge develops on the upper surface and a mild pressure pulse transforms into a very weak suction ridge on the lower surface in a similar way to the clean interaction tests.

The similarity between these results and the clean interaction data indicates that a complete vortex exists before the interaction with the instrumented blade. As the vortex is completely severed by the first interaction, the vortex must reconnect within the space between the two blades. The extent of the similarity of the response suggests that the reconnection process is complete with both rotational and axial velocity components being re-established. This contrasts with the findings of Cary [47], where the vortex was cut at multiple locations along its length but is consistent with the results of Johnston and Sullivan [48] for a near orthogonal interaction although the fate of the axial component, it that case, was not observed. The results presented here indicate that while the vortex cutting process removes significant momentum from the core, complete destruction is avoided. As the vortex strength is expected to increase away from the blade, a pressure gradient will exist along the core that may promote the regeneration of both the axial and rotational flow components.

As may be expected from the pressure data, the normal force and pitching moment coefficient curves exhibit the same basic features as those of the clean interaction case. The most significant difference is a reduction in the severity of the response to the interaction.

\subsubsection{The effect of sub-critical axial flow on the interaction response}

Testing in the larger wind tunnel was conducted over wide range of wind tunnel velocities and vortex generator settings. These are outlined in Table 2 where it may be observed that the different settings also provided a range of axial flow parameters.

Of the cases shown in Table 2 , the $30 \mathrm{~m} / \mathrm{s}$ case is clearly sub-critical and the rest are super-critical. The effect of this can be observed in Fig. 37 where the variations with time of the interactional contribution to the blade normal force coefficients at the vortex centre height for the different free stream velocities are plotted for a blade 
incidence of $6^{\circ}$. These data illustrate well the importance of the axial flow parameter. The most interesting feature in this figure is that the impulsive increment in $(\mathrm{Cn}-\mathrm{Cn} 0)$ for the case of $\mathrm{V}=30 \mathrm{~m} / \mathrm{s}$ is much less than in the other three cases for which the magnitudes of the normal force responses are broadly similar. For the sub-critical flow the pressure waves propagate both upstream and downstream resulting in a more moderate vortex cutting response. Marshall and Krishnamoorthy [50] provided a theoretical estimate of the normal force coefficient response during vortex cutting for sub-critical flows. They noted that the response is characterized by a reduction in normal force in the early stages of the interaction followed by a monotonic rise towards an asymptotic value. This description is in general agreement with the result presented in Fig. 37 at $\mathrm{V}=30 \mathrm{~m} / \mathrm{s}$.

\subsubsection{Interaction measurements on a model tail rotor}

In this section, results are presented for the case of the model tail rotor interacting with the tip vortex from the vortex generator. Since there were only five pressure transducers in each chordal array, it is not possible to present surface pressure distributions of the type shown above. Instead, the pressure time histories for individual transducers are presented and compared for cases with and without an interacting vortex. Once again, in all of the results presented in this section, the lower surface has been defined as the side towards which the vortex axial flow is directed.

The way in which the interaction manifests itself on the upper surface of the rotor blade at a position close to the leading edge $(x / c=0.06, r / R=0.69)$ is shown in Fig. 38 for a blade pitch angle of $2^{\circ}$. In this case, as in all of the cases reported in this section, the free stream velocity was $30 \mathrm{~m} / \mathrm{s}$, the rotational speed of the vortex generator was fixed at 400 revolutions per minute (RPM) and the tail rotor rotational speed was set at 1600 RPM.

In Fig. 38 there are two overlaid measured pressure coefficient variations for four rotations of the tail rotor. One set of data was measured with the vortex generator switched off (dotted line) and the other with it running normally (full line). One of the main features of the figure is the cyclic variation in the level of the pressure coefficient. The relative motion of the tail rotor blade with respect to the free stream velocity causes this. When the tail rotor is below the rotor axis and moving into the oncoming wind the dynamic pressure on the blade is greater than when it is moving above the rotor axis in the same direction as the wind. Another significant feature of the figure is the sharp downward spike in each cycle. This corresponds to the passage of the rotor blade through the wake of its own hub and is accompanied by severe, high frequency, blade flapping. The severity of this is primarily due to the physical scaling of the tail rotor dimensions and its effect on the hub wake. There are no 'self' BVI events apparent in the trace recorded with the vortex generator switched off. This is because the tip vortices from the tail rotor are relatively weak and are carried downstream rather than towards the tail rotor disk by the incoming flow. In the figure the tail rotor interaction with the incoming vortex occurs when the blade is just above centre on the front of the tail rotor disk and is framed by a rectangular box in each plot. The interaction is characterised by a sharp increase in the leading edge suction in a similar manner to that observed during measurements on the stationary blade.

Figure 39 shows the response at the corresponding leading-edge transducer on the lower surface. The previous studies on the stationary blade would suggest that the 
response should be quite different on this side of the blade. In fact, it would be anticipated that, rather than increased suction, the lower surface leading edge would experience a rise in pressure during the interaction due to the axial core flow impinging on the surface. This is indeed the case.

Figure 40 shows pressure coefficient variations measured at two locations on the upper surface of the rotor blade for the same test case as that presented in Figs 38 and 39. The specific locations are both at radial position $\mathrm{r} / \mathrm{R}=0.69$ but lie further down the chord. In fact, comparison of the two plots in this figure with Fig. 38 allows the pressure response due to the chordal progression of the vortex to be observed. It is immediately obvious that, by the time the vortex has progressed to $30 \%$ of chord, the additional suction due to the vortex cutting has diminished substantially. Indeed, at this location only a very small disturbance, in terms of both duration and magnitude, can be detected. Further down the chord at $\mathrm{x} / \mathrm{c}=0.75$ there is no obvious sign of any change in the level of the pressure coefficient compared with the 'no vortex' case. Taken with the other results at this radial position, this suggests that the gross behaviour is similar to that previously measured on the stationary blade.

\section{Numerical modelling of orthogonal blade vortex interaction}

There have been relatively few computational studies of helicopter tail rotor blade vortex interaction and, of these, most have attempted to provide estimates of the acoustic emissions from the tail rotor rather than attempting to model the detail of the interaction itself. One of the first attempts to estimate the acoustic emissions was made by Shlinker and Amiet [45] who studied the phenomenon of vortex cutting both experimentally and numerically. They characterised the noise source as an unsteady loading fluctuation that was estimated using unsteady thin aerofoil theory in combination with a prescribed vortex velocity distribution. Lowson's moving dipole theory [69] was then used to predict the far-field noise. George and Chou [70], and later Tadghigi [71], used an almost identical approach to predict the noise associated with the chopping of a near normally incident vortex by a flat plate. George and Chou used the CAMRAD free wake model to predict the main rotor wake geometry with the simplifying assumption that the tail rotor had no effect on the main rotor wake structure. They then utilised the calculated tip vortex geometry as an input to an idealised vortex formulation used for the interaction simulation. It was found that the relative phasing of the main and tail rotors had a strong influence on the generated noise. It was also recognized, however, that the simulation ignored the effect of axial flow in the vortex core and it was suggested that this could be an important factor in the noise generation process.

Quackenbush et al. [72] and later Yin and Ahmed [73] also employed free wake simulations to predict the aerodynamics of tail rotor blade vortex interactions. The former used a novel technique to extract high-resolution flow information from relatively coarse initial wake simulations. They used this technique to investigate the contributions of the different parts of the main rotor wake to the noise generated by the tail rotor. Yin and Ahmed, on the other hand, used a three-dimensional panel method based free wake simulation to evaluate the unsteady pressure distributions on the blades of the tail rotor. The acoustic response was then calculated using a code 
based on the Ffowcs Williams Hawkings equation [74]. They noted that, in hover, a change in the direction of the tail rotor rotation produced a change in the tonal content and directivity of the noise produced. There was no significant change in the peak noise level however. Also, in contradiction to reported experimental studies, they found no decrease in tail rotor noise levels when operated in the presence of the main rotor. They did obtain qualitative agreement with experiments for climbing flight where they found the tail rotor noise dominated the acoustic emissions from the helicopter. Interestingly, they suggested that noise emissions may be highly configuration specific and that this may account for some of the differences between the predicted acoustic response and previous experiments.

A more recent acoustic simulation study, using a similar approach but based on a high resolution unsteady compressible Euler calculation scheme, has been conducted by Yang et al. [75]. In this work, the computational domain was divided into a series of overlapping grids representing the main rotor, tail rotor, near field and far field domains. The test case used was from a $1 / 7^{\text {th }}$ scale test of the AH-1 helicopter in the DNW wind tunnel [76] and predictions were made for both hovering and forward flight. Interactions of the main rotor tip vortices with the tail rotor were identified in the forward flight computations but were found to be generally less significant than the more frequent 'self' BVIs. It was, however, demonstrated that this depended to some extent on the directivity. Figures $41 \& 42$ show the sound pressure levels from the tail rotor measured at two different locations, both 1000 rotor radii from the main rotor axis. In Fig. 41 interactions of the main rotor wake with the tail rotor are visible in the second and fifth revolutions of the tail rotor and, at this measurement location, produce sound pressure levels that are much lower than the 'self' BVIs. At the second location, Fig 42, the sound pressure levels associated with the two types of interaction are much closer in magnitude. In the future this method will be used to examine a broader range of operationally realistic cases to establish the significance of tail rotor settings on acoustic emissions. This should also allow a more comprehensive understanding of the relationship between 'self' and main rotor tip vortex interactions on the tail rotor disk to be developed.

\subsection{Fluid dynamic simulations of orthogonal blade vortex interaction}

Despite the interest in the prediction of the acoustics of tail rotors, relatively few researchers have focussed on predicting the physical detail of the interaction itself. Computational models of orthogonal blade-vortex interaction have, however, been developed for a wide variety of conditions utilizing several different simplifying approximations. This section first examines inviscid flow studies of the bending of vortex lines around a blade leading edge penetrating into a vortex core, and of the response of a vortex with non-zero axial flow to impulsive chopping of the vortex. Following this, a recent viscous flow computational study that examines the problem of reconnection of vortex lines from the vortex to the blade boundary layer during vortex cutting, along with the subsequent response of a vortex core with non-zero axial flow, will be presented.

\subsubsection{Inviscid flow modelling of orthogonal blade vortex interaction}

When a blade leading edge penetrates into a vortex core, there exists a variety of forces acting on the blade due to a combination of viscous and inviscid effects. The 
inviscid force in the direction of blade motion $U$ was examined in a series of computations by Marshall and Grant [77] using an inviscid vortex method to study impact of a vortex ring on a blade with different impact parameter values. The inviscid force, which acts to pull the blade toward the vortex, is due to the decrease in pressure near the blade leading edge as the blade penetrates into the vortex core, as shown in Fig. 43b. Howe [78,79] showed that under the assumptions of the slenderbody approximation, the drag force exerted on a blade chopping through a vortex with no axial flow should vary with the square of the vortex circulation. Computations of Marshall and Grant from the full inviscid theory with impact parameter values ranging from 0.6 to 3.1 , using a NACA0012 blade having thickness parameter $T=1.6$, yield drag coefficients that scale nearly in proportion to $\Gamma^{2}$, which agrees well with Howe's prediction even though the conditions for the slender-body theory are not satisfied by the airfoil used in these computations. Marshall and Grant argue that this scaling should hold under the more general rapid-distortion approximation, which requires only that the vortex deformation is caused primarily by the potential flow past the blade, rather than by the vortex-induced flow. Computational results for vorticity vectors from this inviscid study are shown in Fig. 43a, which show that the vortex lines deflect around the blade leading edge during penetration, creating a highintensity vorticity sheet surrounding the leading edge.

A study of the inviscid response of a vortex core to impulsive cutting by a blade in an incompressible fluid is reported by Krishnamoorthy and Marshall [50] and, for supercritical vortex flow, by Lee et al. [80]. The average vortex core radius increases on the upstream side and decreases on the downstream side of the blade relative to the vortex jet flow, similar to the experimental results shown in Fig. 16. The computed core radius behind these wave fields is found to compare well with predictions obtained using the plug-flow model for the vortex jet by Marshall [51]. Krishnamoorthy and Marshall show that for subcritical vortices, an upstreampropagating wave forms on the side of the vortex that is compressed by the axial vortex jet flow, as shown in Fig. 44. This wave is characterized by a series of vortex rings of alternating sign. While Krishnamoorthy and Marshall do not observe this upstream-propagating wave field for supercritical vortex flow, a later study of the vortex chopping problem in a compressible fluid by Sheikh and Hillier [81] report upstream-propagating waves with alternating-sign vortex rings for cases well into the supercritical vortex regime. We recall that a supercritical vortex is by definition one where the axial jet flow is sufficiently strong that all linear axisymmetric waves on the vortex core are swept downstream. Certain nonlinear wave forms can remain stationary, or propagate upstream, on the vortex core even under supercritical conditions, as reported by Benjamin [82] and Leibovich and Kribus [83] for axisymmetric solitary waves, although Leibovich and Kribus report occurrence of nonlinear periodic wave trains (with alternating-sign azimuthal vorticity) only for subcritical flow. Sheikh and Hillier report that the fluid compressibility in their study causes a local decrease in the axial flow parameter (which they term the "normal impact parameter") close to the blade, which could explain their observation of upstream-propagating waves for cases where such waves are not observed for incompressible vortex flows. A physical explanation of the vorticity dynamics leading to formation of the alternating-sign vortex rings is also given by Sheikh and Hillier, which involves a combination of reorientation of initially axial vorticity in the radial direction by the jet flow as it impinges on the plate, and then tilting of this vorticity in the azimuthal direction by a radial gradient in the vortex azimuthal velocity. 


\subsubsection{Viscous flow modelling of orthogonal blade vortex interaction}

A computation of the full viscous orthogonal vortex cutting problem has only recently been reported by Liu and Marshall [84]. This computation was performed using a block-structured finite-volume method on a rectangular domain (Fig. 45), with periodic boundary conditions on the top and bottom surfaces and symmetry boundary conditions on the side surfaces. The computations were performed in the frame of the blade, where the steady flow past the blade was first computed and then the columnar vortex with a given axial velocity was input upstream of the blade. Values reported for core radius and axial velocity were measured at the time of initial vortex impact on the vortex outer core.

Typical results for cutting of a supercritical vortex are given in Fig. 46, which shows contours of the vertical vorticity component $\omega_{z}$ at four different times during the cutting process for a case with large downward axial flow $(A=3.8)$ and large impact parameter $(I=3.8)$. Prior to impact of the blade onto the vortex core, we observe positive $\omega_{z}$ within the vortex core and negative $\omega_{z}$ within the blade boundary layer. As the blade leading edge penetrates into the core, these two regions of vorticity cross-diffuse, leading to reconnection of the vortex lines in the vortex to those in the blade boundary layer. However, as the vortex core center passes over the blade leading edge, the velocity induced by the vortex along the blade span changes direction, which has the effect of reversing the sign of $\omega_{z}$ within the blade boundary layer near the leading edge. This sign reversal cuts off the vorticity cross-diffusion, consequently halting the vortex line reconnection process. The consequence of these events can be seen in Fig. 47, where we show the vortex lines for a case with no axial flow $(A=0)$ at the same time as in Figure 46d. In this figure, the vortex lines on the right-hand side of the vortex (to the right of the vertical line on the blade), have reconnected with those in the blade boundary layer and deflect rearward into the blade wake. The vortex lines to the left of this vertical line have not reconnected, and instead deflect around the blade leading edge and continue upward into the upper section of the vortex core. Shortly after the reversal in spanwise velocity along the leading edge has occurred and all of the vorticity $\omega_{z}$ within the region of the boundary layer near the leading edge has become positive, the reconnection process is halted and all remaining vortex lines deflect around the blade nose as the blade penetrates further into the vortex core.

Also associated with the vortex cutting process is a chopping of the vortex axial flow, resulting in an increase in core radius (and decrease in vorticity magnitude) in the upper section of the vortex and a decrease in core radius (and increase in vorticity magnitude) in the lower section of the vortex. In Fig. 46 it may be observed that the increase in vorticity within the lower section of the vortex causes separation of the blade boundary layer on the side of the vortex that opposes the free-stream velocity. Interestingly, boundary layer separation is not observed at an earlier time (e.g., Fig. $46 \mathrm{~b})$ as the blade leading edge penetrates into the axial jet within the vortex core.

The effect of vortex Reynolds number on the decrease in positive circulation $\Gamma^{+}$and axial flow $Q$ in the blade centerplane $z=0$ is examined in Fig. 48 for cases with $\operatorname{Re}_{V}=73,245$, and 488, where $\Gamma^{+}$is obtained by integrating $\omega_{z}$ over all regions of 
the blade centerplane where $\omega_{z}>0$. Both $\Gamma^{+}$and $Q$ are approximately constant until the blade leading edge begins penetrating into the outer part of the vortex core at about $t c / U=0.5$. After this point both $\Gamma^{+}$and $Q$ decrease rapidly until about $t c / U=1.0$, at which point the blade has penetrated nearly completely through the vortex core. Neither quantity exhibits significant influence of Reynolds number within the range of $\mathrm{Re}_{v}$ examined.

The chopping of the vortex axial flow produces an asymmetry across the blade centerplane, which leads to increased pressure on the upper blade surface and decreased pressure on the lower blade surface. The difference between the pressure gradient for the case shown in Fig. 46 and the same case with no axial flow is shown in Fig. 49a. In this plot the blade surface is opened out, such that the leading edge is a vertical line in the center, the upper surface is to the left of this line, and the lower surface is to the right of this line. It is observed that the principal pressure differences caused by this asymmetry occur within the vortex cores. The consequence of this pressure difference is a net (lift) force acting on the blade in the direction of the vortex axial velocity. One measure of this lift force, which is convenient for experimental studies, is to integrate the pressure force over a section of the blade, corresponding to a line $y=$ const in Fig. 49a, which when nondimensionalized yields the sectional lift coefficient $C_{n}$. In Fig. 49b, we show a comparison between the value of $C_{n}$ obtained experimentally by Wang et al. [63] (symbols) and the computed values (solid line) for a slice $y=0$ (passing through the vortex core). All parameters for the two cases were the same except for the Reynolds number, where $\mathrm{Re}_{V}=44$ for the computations and $\mathrm{Re}_{V}=51,000$ in the experiments. The maximum value of $C_{n}$ in the predictions agrees reasonably closely with the experimental value, despite the large difference in Reynolds number. We do notice that the experimental value increases more rapidly in time than the computations, which is likely due to an increased amount of inviscid deflection of the vorticity around the blade leading edge for the higher Reynolds number experiments than observed in the computations.

Computations with various values of the parameters $A, I, T$ and $\operatorname{Re}_{v}$ indicate that the lift force (obtained by integrating pressure force over the entire blade surface) is not sensitive to either $T$ or $\operatorname{Re}_{v}$ and that it increases linearly with both $A$ and $I$. These results suggest that the computations can be correlated by plotting the parameter $C_{L}^{\prime \prime} \equiv L /\left(\pi \rho a^{2} w_{0} U\right)$ as a function of dimensionless time. This correlation is shown in Fig. 50 to collapse all of the computational data to within about $\pm 30 \%$.

It is interesting that the correlation in Fig. 50 does not depend on the vortex circulation, but rather only on the axial velocity and impact velocity. Much of the emphasis in the helicopter aerodynamics community has focused on accurate prediction of rotor vortex circulation and on preventing erosion of the vortex circulation by numerical dissipation during the computations. By contrast, the result in Fig. 50 suggests that accurate prediction of the vortex core radius and vortex axial velocity is critical in order to accurately predict the impulsive force on the blade during OBVI. This observation may have significant influence on methods used to predict helicopter vortices, since the net vortex circulation is relatively insensitive to the turbulence surrounding the vortex, whereas both vortex core radius and axial 
velocity can be significantly influenced by the turbulence both inside and surrounding the vortex core (Ragab and Sreedhar, [85]).

\section{Concluding Remarks}

Unlike many fluid dynamic phenomena, helicopter tail rotor interaction has been the subject of research for less than thirty years. Initially, studies focused very much on the solution of related operational problems with design developments being based, to some extent, on trial and error. Only recently has the detail of the interaction process been clarified through a series of computational and experimental studies.

Orthogonal BVI is an important limiting case of the tail rotor interaction phenomenon as it is manifest when a helicopter is in forward climbing flight. This is the flight condition where tail rotor noise dominates the emission spectrum. Orthogonal BVI has particular characteristics that distinguish it from other types of interaction. In particular, it is the only type of interaction where the dominant parameter is the axial flow within the vortex rather than its rotational component. Since calculation of the axial flow within a tip vortex is more difficult than estimation of the circulation strength, prediction of even the isolated interaction response is problematic. When the entire helicopter flow field is considered the complexity of the problem increases considerably. If, however, realistic numerical or empirical representations of tip vortex axial flow can be incorporated into contemporary rotor prediction codes, a basis for predicting the response during orthogonal interaction will have been established. In addition to the prediction methods presented here, work currently underway at the University of Glasgow suggests that indicial modelling, of the kind routinely used in unsteady rotor airload calculations [86], may provide a fast and reliable means of predicting the unsteady interactional response.

In the longer term, it is clear that the key to accurate prediction of tail rotor acoustics is that all of the interactional aerodynamics have to be captured within the modelling strategy. An illustration of this is that although the interaction of the tail rotor blade with a main rotor tip vortex results in an undesirable acoustic emission, the interaction of the main rotor wake with the tail rotor wake may produce a beneficial effect through the amelioration of tail rotor 'self' BVIs. As yet, the precise mechanism for this phenomenon is unclear and further experimental investigation of it would, therefore, be desirable. This would also support the development of more comprehensive flow simulations that, although computationally expensive, are becoming more feasible with each passing year. 


\section{Acknowledgements}

Research support for J. Marshall was provided by Hitachi Industries, Ltd., and by the U.S. Army Research Office under grant number DAAH04-96-1-0081. The work at Glasgow was supported by the U.K. MoD, Westland Helicopters, the Engineering and Physical Sciences Research Council and the European Research Office of the U.S. Army under grant N68171-00-M-5988.

The authors wish to acknowledge the substantial contributions of Dr. C. Copland, Dr. C. Doolan, Prof. T. Wang and Ms. J. Early to the work carried out at the University of Glasgow.

\section{References}

1. Lugt HJ, Vortex Flow in Nature and Technology, John Wiley and Sons, ISBN No. 0-471-86925-2, 1983.

2. Chigier NA, Corsiglia VR, Tip Vortices - Velocity Distributions, NASA TM X62,087, 1971.

3. Newman BG, Flow in a Viscous Trailing Vortex. Aeronautical Quarterly 1959;10:149-162

4. Rorke JB, Moffett RC, Measurement of Vortex Velocities Over a wide Range of Vortex Age, Downstream Distance and Free Stream Velocity. NASA CR 145213, 1977.

5. Brocklehurst A, Main Rotor/Tail Rotor Interactions Near Hover, Westlands Research Memorandum 535, 1986.

6. Beddoes TS, A Wake Model for High Resolution Airloads, Proceedings of the $2^{\text {nd }}$ Int. Con. On Basic Rotorcraft Research, Triangle Park, NC, 1985

7. Ellin ADS, An In-flight Experimental Investigation of Helicopter Main Rotor/Tail rotor Interactions, PhD Dissertation, University of Glasgow, 1993

8. Krishnamoorthy S, Marshall JS, Three-dimensional Blade-vortex Interaction in the Strong Vortex Regime, Physics of Fluids 1998;10(11):2828-2845

9. Norman TR, Light JS, Rotor Tip Vortex Geometry Measurements Using the Wide Field Shadowgraph Technique, AIAA paper 86-1780. In: Proceedings of the $4^{\text {th }}$ AIAA Applied Aerodynamics Conference, San Diego, 1986

10. Sheridan PF, Smith RP, Interactional Aerodynamics - a New Challenge to Helicopter Technology, Journal of the American Helicopter Society, 1980;25(3)

11. Surendraiah M, An Experimental Study of Rotor Blade-vortex Interaction, MS Thesis, University of Pennsylvania, 1969

12. Padakannaya R, Experimental Study of Rotor Unsteady Airloads Due to Bladevortex Interaction, NASA CR-1909, 1971

13. Caradonna FX, Lautenschlager JL, Silva MJ, An Experimental Study of Rotorvortex Interactions, AIAA Paper88-0045, 1988

14. Horner MB, Saliveros E, Kokkalis, A, Galbraith, RAMcD, Results from a Set of Low Speed Blade-vortex Interaction Experiments, Experiments in Fluids 1993;14:341-352

15. Renzoni P, Mayle, RE, Incremental Force and Moment Coefficients for a Parallel Blade-vortex Interaction, AIAA Journal, 1991:29:6-13 
16. Wittmer KS, Devenport WJ, Rife MC, Glegg SAL, Perpendicular blade vortex interaction, AIAA Journal, 1995;33(9):1667-1674

17. Wittmer KS, Devenport WJ, Effects of Perpendicular Blade Vortex Interaction. Part 1: Turbulence Structure and Development, AIAA Journal, 1999;37(7):805812

18. Wittmer KS, Devenport WJ, Glegg SAL, Effects of Perpendicular Blade Vortex Interaction. Part 2: Parameter Study, AIAA Journal, 1999;37(7):813-817

19. Liou S, Komerath N, McMahon H, Measurement of Transient Vortex-Surface Interaction Phenomena, 27 ${ }^{\text {th }}$ AIAA Aerospace Sciences Meeting, Reno, 1989

20. Leishman JG, Bi N-P, Aerodynamic interactions between a rotor and a fuselage in forward flight, Journal of the American Helicopter Society, 1990;35(3):22-31

21. Bi N-P, Leishman JG, Crouse GL, Investigation of Rotor Tip Vortex Interactions with a Body, AIAA Journal of Aircraft, 1993;30(6):879-888

22. Affes H, Conlisk AT, Model for Rotor Tip Vortex-Airframe Interaction, Part 1: Theory, AIAA Journal, 1993;31(12):2263-2273

23. Cook CV, A Review of Tail Rotor Design and Performance, Vertica, 1978;2:163181

24. Smith AC, Review of Helicopter Low Speed Handling Problems Associated with the Tail Rotor, RAE TM FS(b) 683, 1987

25. Newman SJ, The Foundations of Helicopter Flight, Edward Arnold Press, 1994, ISBN 0340587024

26. Leverton JW, Pollard JS, Wills CR, Main Rotor Wake/Tail Rotor Interaction, Vertica, 1977;1:213-221

27. Leverton JW, Pike TC, The Importance of Tail Rotor Interaction as an Acoustic Source. In: Proceedings Of the $49^{\text {th }}$ American Helicopter Society Forum, St. Louis, 1993. p. 73-88

28. Samokhin VF, Impulsive Noise of the Helicopter Tail Rotor. In: Proceedings of the $21^{\text {st }}$ European Rotorcraft Forum, St. Petersburg, 1995

29. Jacobs EW, Mancini J, Visintainer JA, Jackson TA, Acoustic Flight Test Results for the Sikorsky S-76 Quiet Tail Rotor at Reduced Tip Speed. In: Proceedings of the 53 ${ }^{\text {rd }}$ American Helicopter Society Forum, Virginia Beach, 1997. p. 23-41

30. Leverton JW, Reduction of Helicopter Noise by use of a Quiet Tail Rotor. In: Proceedings of the $6^{\text {th }}$ European Rotorcraft Forum, 1981

31. Baldwin SF, Handley CS, Flight Tests to Explore Tail Rotor Limitations in the Low Speed Envelope. In: Proceedings of the $14^{\text {th }}$ European Rotorcraft Forum, 1988

32. Brotherhood P, The Determination of Helicopter Blade Local Normal Force Coefficient, Incidence and Stall Boundaries from Flight Measurements of Leading and Trailing Edge Pressures, DRA Technical Memorandum, 1992.

33. Martin RM, Burley CL, Elliott, JW, Acoustic Test Of A Model Rotor And Tail Rotor: Results For The Isolated Rotor And Combined Configuration, NASA TM 101550, 1989

34. Bhagwat MJ, Leishman JG, Generalized viscous vortex model for application to free-vortex wake and aeroacoustic calculations. In: Proceedings of the $58^{\text {th }}$ Annual forum of the American Helicopter Society, Montreal, 2002

35. McAlister KW, Heineck JT, Measurements of the Early Development of Trailing Vorticity from a Rotor, NASA/TP-2002-211848, 2002

36. Doolan CJ, Coton FN, Galbraith RAMcD, Surface Pressure Measurements of the Orthogonal Vortex Interaction, AIAA Journal, 2001;39(1):88-95 
37. Doolan CJ, Coton FN, Galbraith, RAMcD, The effect of a preceding blade on the orthogonal vortex interaction, Journal of the American Helicopter Society, 2001;46(3):221-227

38. White RP, Balcerak JC, Pegg RJ, A Parametric Model Study of the Noise Generated by the Aerodynamic Interaction of the Tail Rotor with the Wake of the Main Rotor. In: AHS Mid-East Region Symposium on Rotor Technology, Essington, 1976

39. Pegg RJ, Shidler, PA, Exploratory Wind Tunnel Investigation of the Effect of the Main Rotor Wake on Tail Rotor Noise, NASA CP-2052, Pt. 1, 1978. p. 205-219

40. Tadghighi H, and Cheeseman IC, A Study of Helicopter Rotor Noise, with Special Reference to Tail Rotors, using an Acoustic Wind Tunnel, Vertica, 1983;7(1):9-32

41. Sternfeld HJr, Schaeffer EG, The Role of Wind Tunnel Models in Helicopter Noise Research. In: Proceedings of the $42^{\text {nd }}$ American Helicopter Society Forum, Washington, June, 1986. p. 513-523

42. Fitzgerald J, Kohlhepp F, Research Investigation of Helicopter Main Rotor/Tail Rotor Interaction Noise, NASA CR 4143, May, 1988

43. Schultz KJ, Splettstoesser WR, Model Tail Rotor Noise Study in the DNW Measured Acoustics, Blade Pressures, Noise Predictions. In: Proceedings of the $18^{\text {th }}$ European Rotorcraft Forum, Arles, September, 1992

44. Schultz KJ, Splettstoesser WR, Helicopter Main Rotor/Tail Rotor Noise Radiation Characteristics from Scaled Model Rotor Experiments in the DNW. In:

Proceedings of the $49^{\text {th }}$ American Helicopter Society Forum, St. Louis, May, 1993

45. Shlinker RH, Amiet RK, Rotor Vortex Interaction Noise, AIAA Paper 83-0720. In: Proceedings of the $8^{\text {th }}$ AIAA Aeroacoustics Conference, Atlanta, 1983

46. Ahmadi A, An experimental investigation of blade-vortex interaction at normal incidence, AIAA Journal of Aircraft, 1986;23(1):47-55

47. Cary CM, An Experimental Investigation of the Chopping of Helicopter Main Rotor Tip Vortices by the Tail Rotor, Part 2: High Speed Photographic Study, NASA CR 177457, Sept., 1987

48. Johnston RT, Sullivan JP, Unsteady wing surface pressures in the wake of propeller, AIAA Paper 92-0277, 1992

49. Krishnamoorthy S, Marshall JS, An experimental investigation of 'vortex shocks', Physics of Fluids, 1994;6(11):3737-3741

50. Marshall JS, Krishnamoorthy S, On the instantaneous cutting of a columnar vortex with non-zero axial flow, Journal of Fluid Mechanics, 1997;351:41-74

51. Marshall JS, Vortex cutting by a blade. Part I. General theory and a simple solution, AIAA Journal, 1994;32(6):1145-1150

52. Marshall JS, Inviscid Incompressible Flow, John Wiley \& Sons, New York, 2001, pp. 283-288

53. Weigand A, The response of a vortex ring to a transient, spatial cut, Ph.D. dissertation, University of California, San Diego, 1993

54. Marshall JS, Models of Secondary Vorticity Evolution during Normal VortexCylinder Interaction, AIAA Journal, 2002;40(1):170-172

55. Marshall JS, Brancher P, Giovannini A, Interaction of unequal anti-parallel vortex tubes, Journal of Fluid Mechanics, 2001;446:229-252

56. Sun M. and Marshall, JS, A flow visualization study of vortex interaction with the wake of a sphere, Journal of Fluids Engineering, 2000;122:560-568 
57. Copland C, Coton FN, Galbraith RAMcD, A study of helicopter tail rotor interaction; Phase 1 - proof of concept. In: Proceedings of the 24th European Rotorcraft Forum, Marseilles, France, 1998

58. Copland C, Methods of Generating Vortices in Wind Tunnels, Ph.D. thesis, Department of Aerospace Engineering, University of Glasgow, 1997

59. Hess JL, Smith, AMO, Calculations of Potential Flow About Arbitrary Bodies, Progress in the Aeronautical Sciences, 1966;8:1-139

60. Doolan C, Coton FN, and Galbraith RAMcD, Measurement of Three-Dimensional Vortices using a Hot Wire Anemometer," AIAA Paper No: 99-3810. In Proceedings of the 30th AIAA Fluid Dynamics Conference, Norfolk,VA, 1999

61. Doolan C, Coton FN, and Galbraith RAMcD, Three-Dimensional Vortex Interactions with a Stationary Blade, The Aeronautical Journal, 1999;103,(1030):579-587

62. Doolan CJ, Green RB, Coton, FN, Galbraith, RAMcD, The orthogonal bladevortex interaction experimental programme at the University of Glasgow. In Proceedings of the 26th European Rotorcraft Forum, The Hague, Sept. 2000

63. Wang T, Doolan CJ, Coton FN Galbraith, RAMcD, Experimental Study of the Three-Dimensionality of Orthogonal Blade Vortex Interaction, AIAA Journal, 2002;40(10):2037-2046

64. Coton FN, Galbraith RAMcD, Wang T, Newman SJ, A Wind Tunnel Based Study of Helicopter Tail Rotor Blade Vortex Interaction, The Aeronautical Journal, 2004;108(1083):237-244

65. Green RB, Doolan CJ, Cannon, RM, Measurements of the orthogonal bladevortex interaction using a particle image velocimetry technique, Experiments in Fluids, 2000;29:369-379

66. Lundgren TS, Ashurst WT, Area-varying waves on curved vortex tubes with application to vortex breakdown, Journal of Fluid Mechanics, 1989;200:283-307

67. Early JM, Green RB, Coton FN, Flow visualisation of the orthogonal blade-vortex interaction using particle image velocimetry, The Aeronautical Journal, 2001;106(1057):137-145

68. Masson CA, Green RB, Galbraith RAM, Coton FN, Experimental investigation of a loaded rotor blade's interaction with a single vortex, The Aeronautical Journal, 1998;102(1018):451-457

69. Lowson MV, The Sound Field for Singularities in Motion, Proceedings of the Royal Society of London, Series A, 1965;286

70. George AR, Chou, ST, Helicopter Tail Rotor Blade-Vortex Interaction Noise, NASA CR 183178, 1987

71. Tadghighi H, An Analytical Model for Prediction of Main Rotor/Tail Rotor Interaction Noise, AIAA paper 89-1130, April 1989

72. Quakenbush TR, Bliss DB, Mahajan A, High Resolution Flow Field Prediction for Tail Rotor Aeroacoustics. In Proceedings of the $45^{\text {th }}$ American Helicopter Society Forum, Boston, May, 1989

73. Yin JP, Ahmed SR, Helicopter Main Rotor/Tail Rotor Interaction, Journal of the American Helicopter Society, 2000;45:293-302

74. Ffowcs-Williams JE, Hawkings DL, Sound generation by turbulence and surfaces in arbitrary motion, Philosophical Transactions of the Royal Society A,1969;264(1151):321-344

75. Yang C, Aoyama T, Saito S, Numerical analysis of interaction noise between main rotor and tail rotor of helicopter. In: Proceedings of the $24^{\text {th }}$ International Congress of the Aeronautical Sciences, Japan, Sept. 2004 
76. Boxwell DA, Schmitz FH, Splettstoesser WR, Schults KJ, Helicopter model rotorblade vortex interaction impulsive noise: scalability and parametric variations, J. of American Helicopter society, 1987;32(1):3-12

77. Marshall JS, Grant JR, Penetration of a blade into a vortex core: vorticity response and unsteady blade forces, Journal of Fluid Mechanics, 1996;306:83-109

78. Howe MS, Contributions to the theory of sound production by vortex-airfoil interaction, with application to vortices with finite axial velocity defect, Proceedings of the Royal Society of London A, 1988:420:157-182

79. Howe MS, On unsteady surface forces, and sound produced by the normal chopping of a rectilinear vortex, Journal of Fluid Mechanics, 1989;206:131-153

80. Lee JA, Burggraf OR, Conlisk AT, On the impulsive blocking of a vortex-jet, Journal of Fluid Mechanics, 1998;369:301-331.

81. Sheikh AH, Hillier R, The compressible perpendicular interaction of a columnar vortex-jet with a flat plate, AIAA Paper 2001-2846, 2001

82. Benjamin TB, Some developments in the theory of vortex breakdown, Journal of Fluid Mechanics, 1967;28:65-84

83. Leibovich S, Kribus A, Large-amplitude wavetrains and solitary waves in vortices, Journal of Fluid Mechanics, 1990;216:459-504

84. Liu X, and Marshall JS, Blade penetration into a vortex core with and without axial core flow, Journal of Fluid Mechanics, 2004; 519: 81-103

85. Ragab S, Sreedhar M, Numerical simulation of vortices with axial velocity deficits, Physics of Fluids, 1995;7(3):549-558

86. Leishman JG, and Beddoes TS, A Semi-Empirical Model for Dynamic Stall, Journal of the American Helicopter Society, 1989:34(3) 


\begin{tabular}{|c|c|}
\hline$a / c$ & $0.065 \pm 0.031$ \\
\hline$\Gamma /(\Omega R a)(\mathrm{PIV})$ & $0.144 \pm 0.022$ \\
\hline$\Gamma /(\Omega R a)$ (Hot wire) & $0.120 \pm 0.044$ \\
\hline$w_{o} / V_{\infty}$ & $0.4 \pm 0.12$ \\
\hline
\end{tabular}

Table 1: A summary of vortex parameters in the small wind tunnel.

\begin{tabular}{ccccc}
\hline \hline$V(\mathrm{~m} / \mathrm{s})$ & 20 & 30 & 40 & 50 \\
\hline $\begin{array}{c}\text { Rotor speed } \\
\text { (r.p.m.) }\end{array}$ & 168 & 252 & 336 & 420 \\
$\begin{array}{c}\text { Vortex height } \\
(\mathrm{mm})\end{array}$ & 980 & 960 & 920 & 900 \\
$a(\mathrm{~mm})$ & $74.18 \pm 16.35$ & $84.83 \pm 33.31$ & $76.30 \pm 10.24$ & $90.30 \pm 49.32$ \\
$\mathrm{Max} V_{\theta}(\mathrm{m} / \mathrm{s})$ & $2.77 \pm 0.35$ & $3.20 \pm 0.45$ & $3.17 \pm 0.38$ & $1.72 \pm 0.41$ \\
$w_{0}(\mathrm{~m} / \mathrm{s})$ & $1.62 \pm 0.23$ & $1.27 \pm 0.23$ & $1.91 \pm 0.25$ & $2.20 \pm 0.20$ \\
$\Gamma\left(\mathrm{m}^{2} / \mathrm{s}\right)$ & $0.90 \pm 0.26$ & $1.26 \pm 0.41$ & $1.26 \pm 0.25$ & $0.77 \pm 0.27$ \\
$T$ & 0.556 & 0.486 & 0.541 & 0.457 \\
$I$ & 10.36 & 12.69 & 15.22 & 36.84 \\
$A$ & 0.84 & 0.54 & 0.73 & 1.62 \\
\hline \hline
\end{tabular}

Table 2: Vortex parameters used during testing in the large wind tunnel 


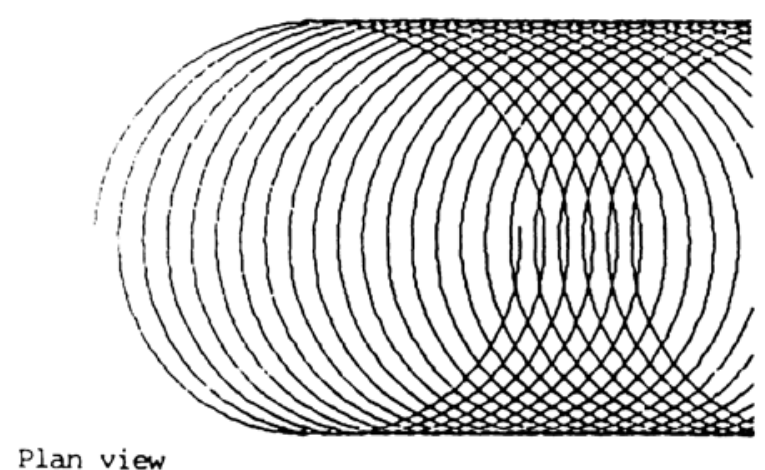

Plan view

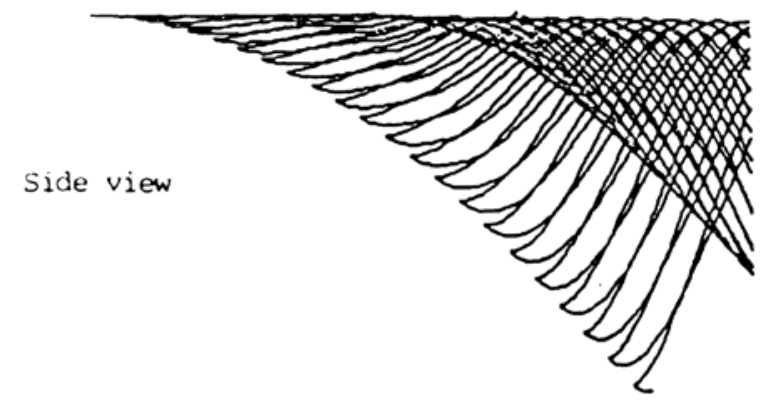

Rear (3/4) view from above disc plane

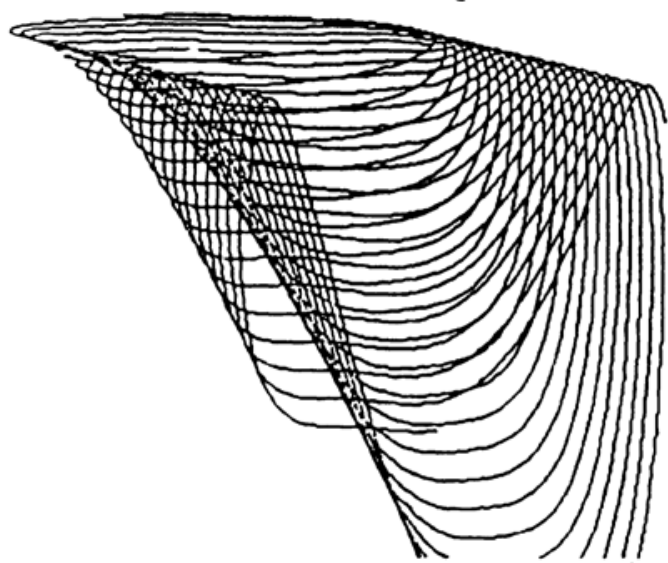

Figure 1. Idealised prescribed wake structure produced by the method of Beddoes [6] for low speed forward flight at advance ratio 0.07 


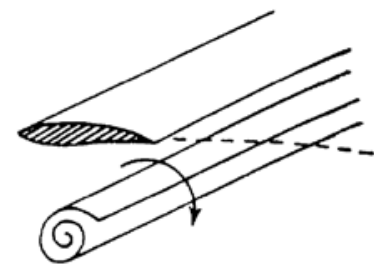

(a)

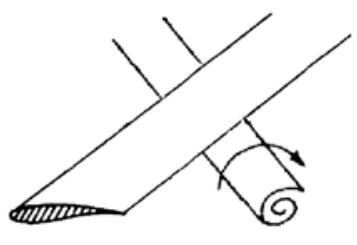

(b)

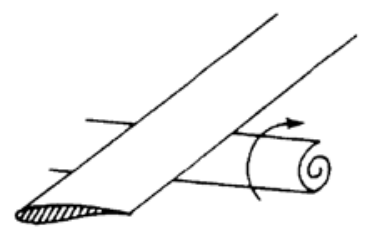

(c)

Figure 2 Types of helicopter main rotor blade vortex interaction (a) parallel, (b) oblique and (c) perpendicular or grazing 


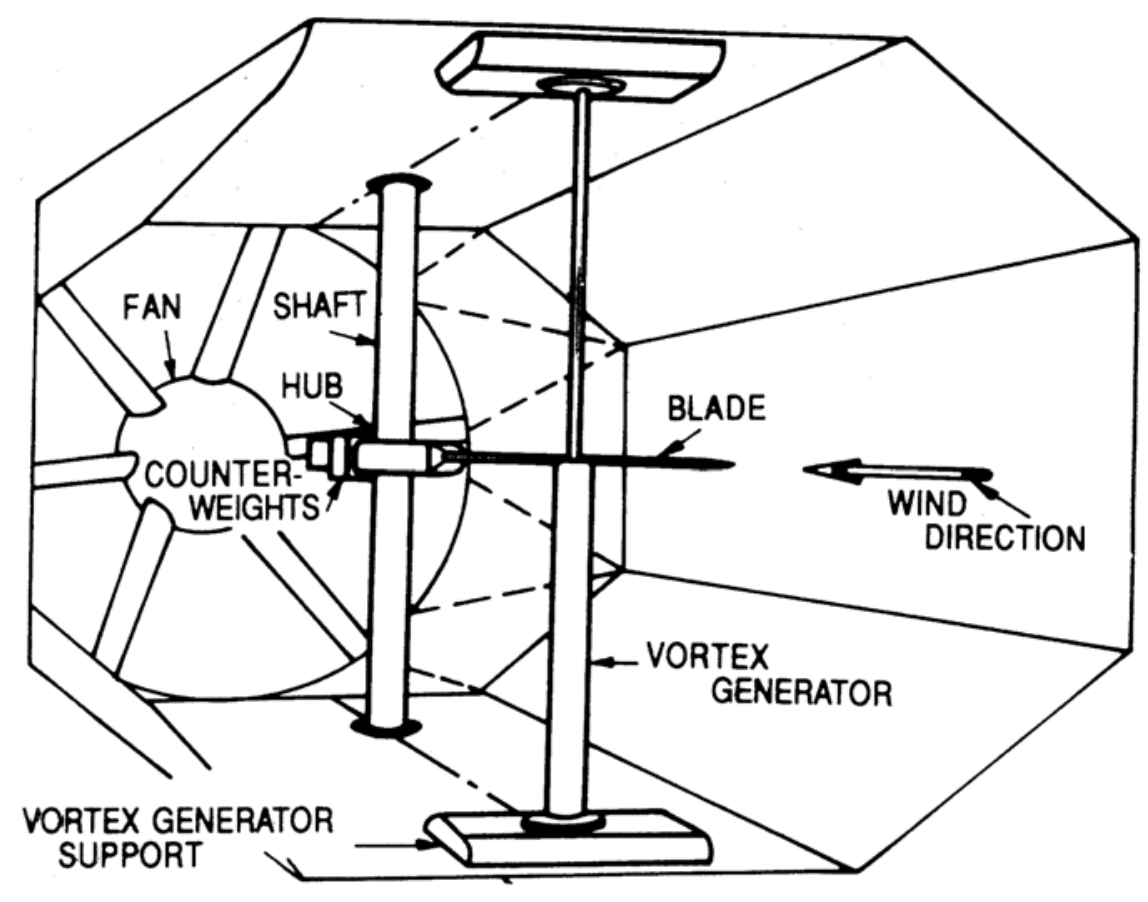

Figure 3. Test set-up used by Horner et al. [14] to study parallel and oblique BVI in which a vortex generated at the intersection of the blades of the vortex generator interacts with the rotor located downstream. 


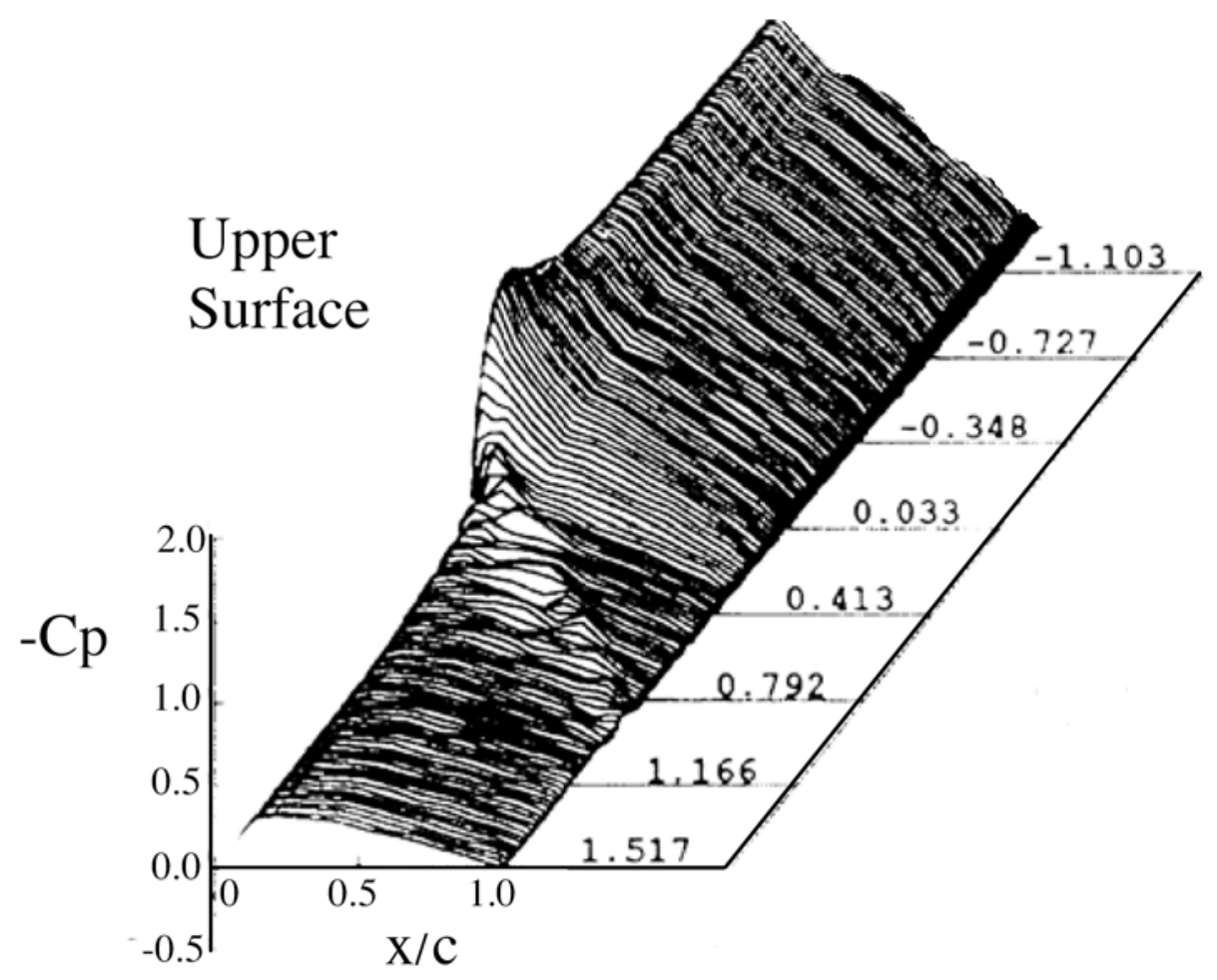

Figure 4 Measured upper surface pressure response during 'head-on' parallel BVI [14] 


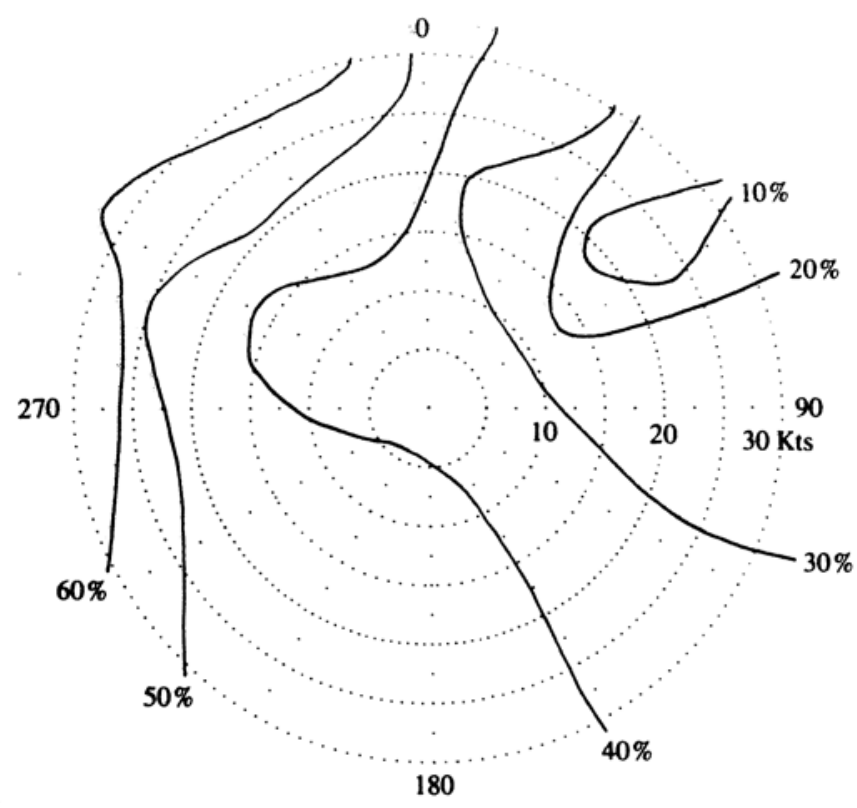

Figure 5. Yaw control margin versus relative wind speed and direction for a Lynx AH Mk5 helicopter [7] 


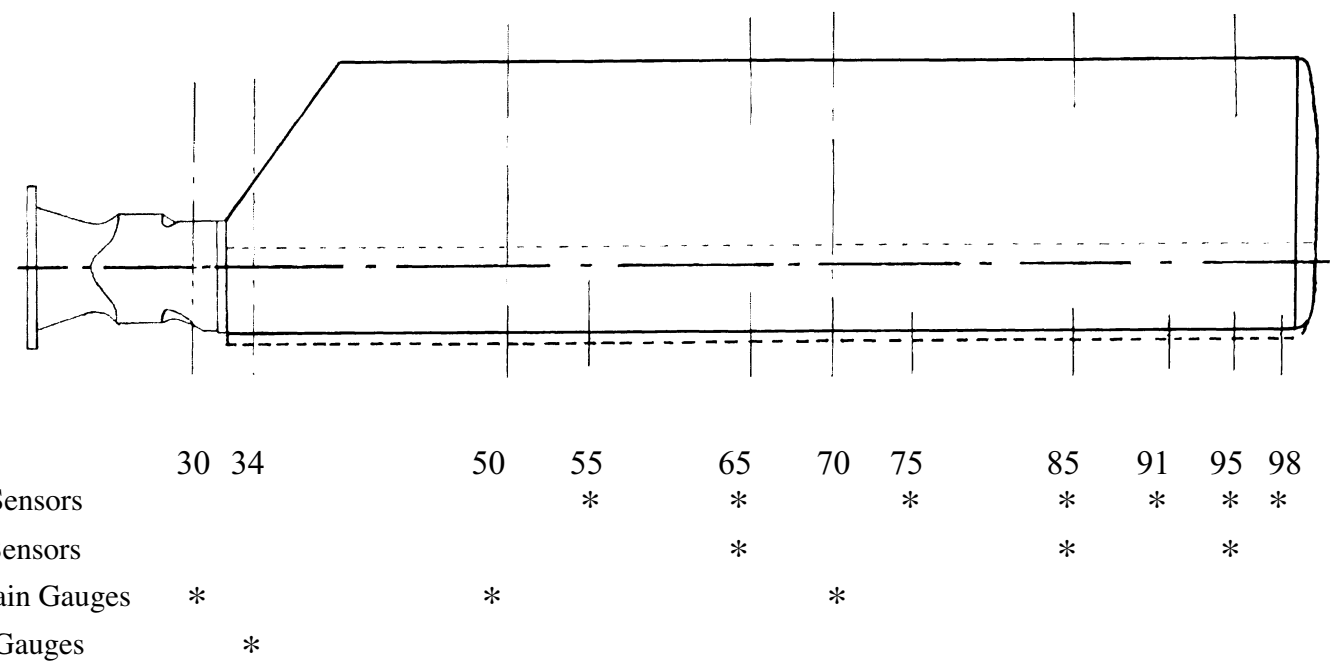

Figure 6. Layout of instrumentation on Westland Lynx tail rotor blade used to study tail rotor interaction during flight tests [7] 


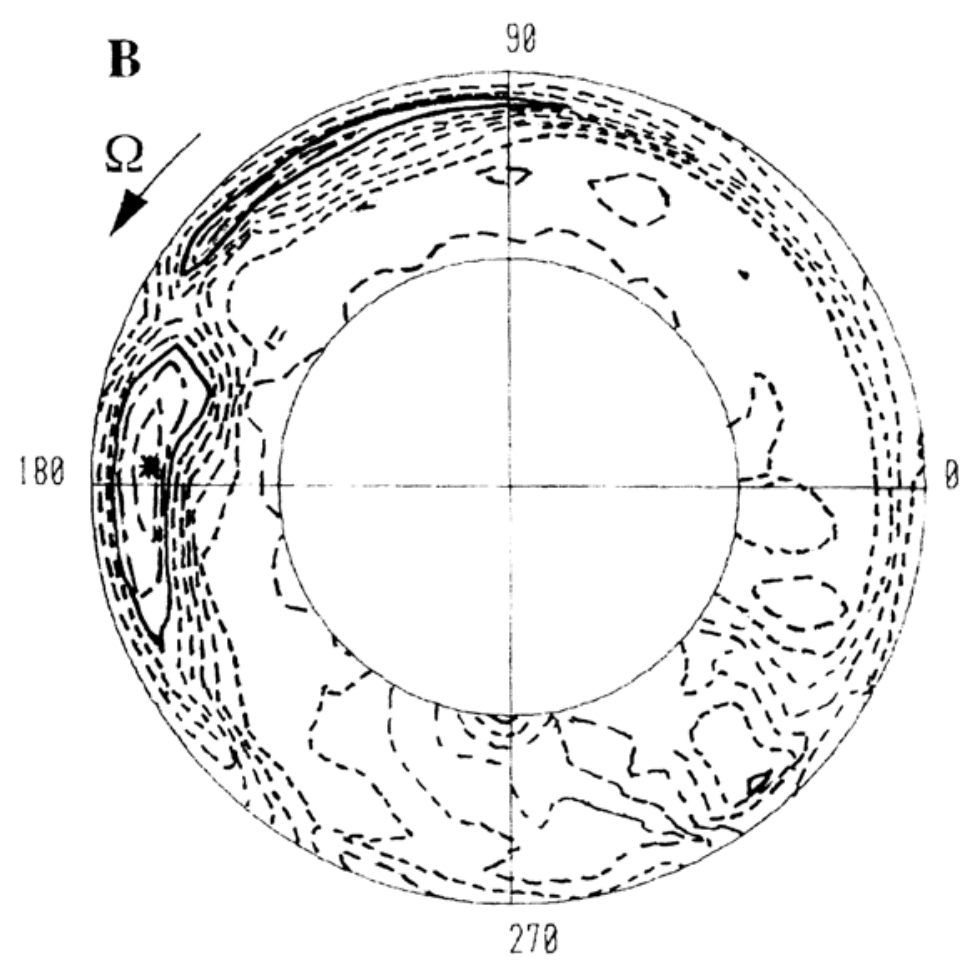

Figure 7 Contours of leading edge pressure coefficient on the tail rotor of a Westland Lynx helicopter in forward flight [7]. An impulsive interaction with a main rotor tip vortex is highlighted just after $180^{\circ}$ by the asterisk. 


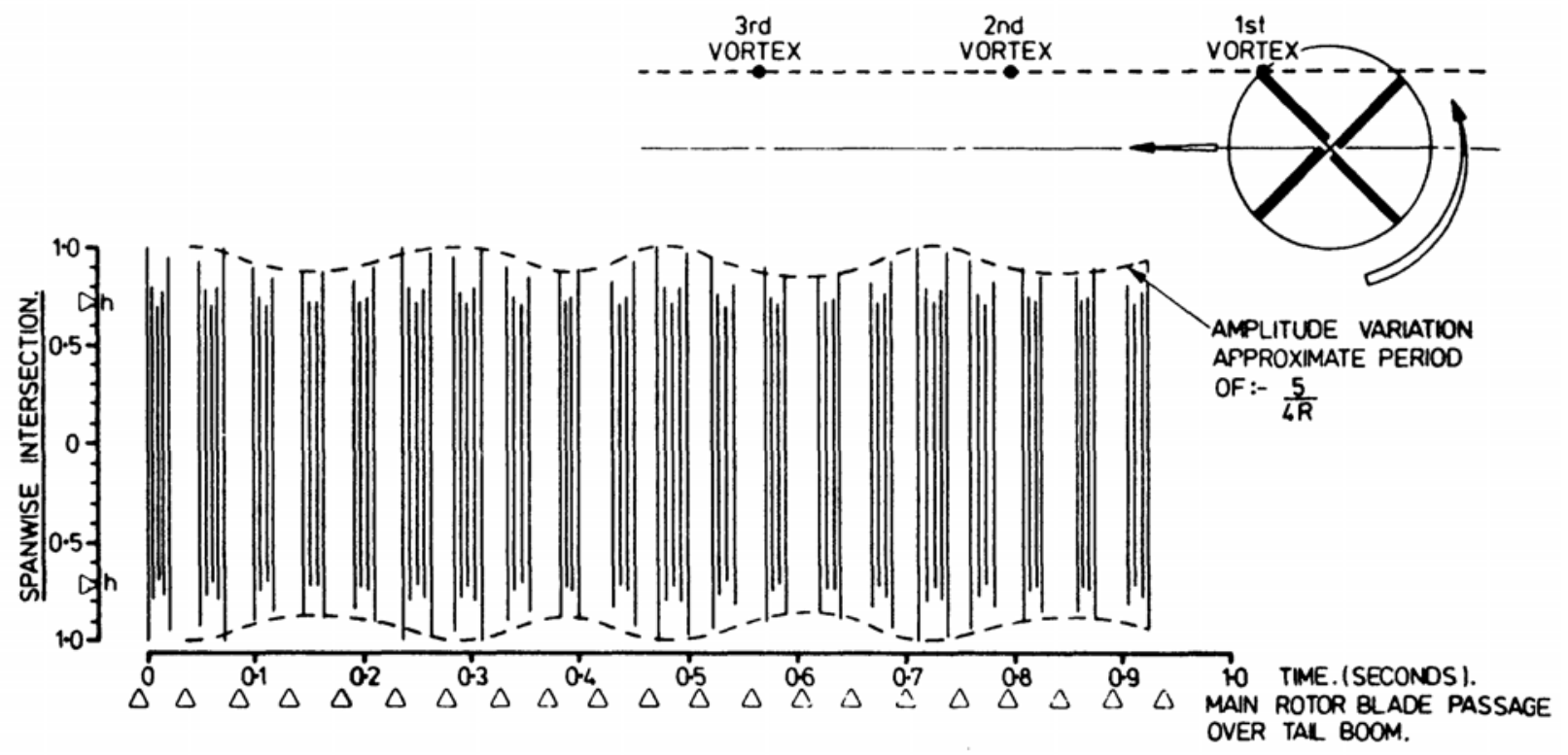

Figure 8 Schematic of helicopter main rotor tip vortex interaction with tail rotor and corresponding intersection envelope [26] 


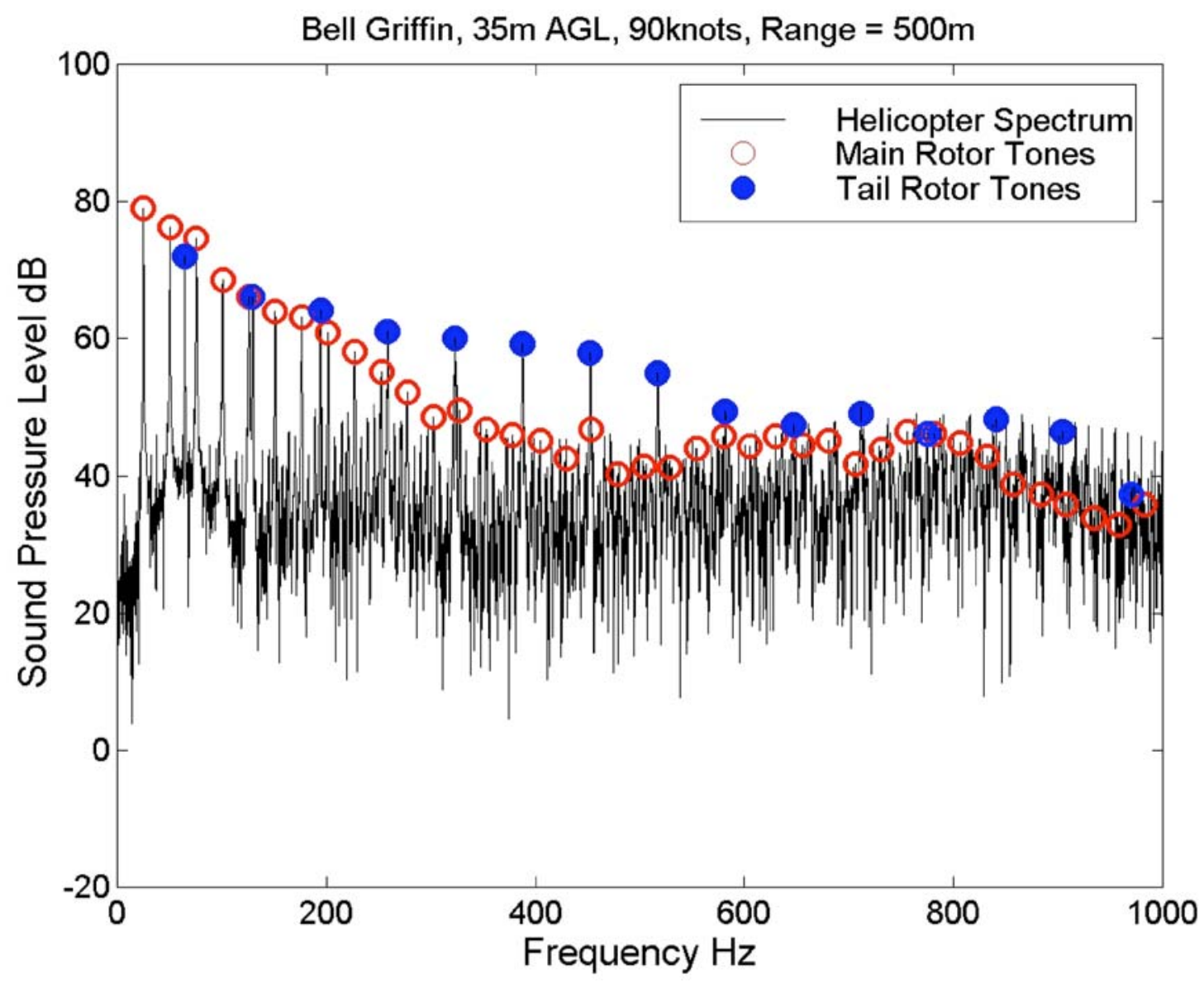

Figure 9. Typical noise spectrum from a helicopter in forward flight highlighting both the main and tail rotor tones. 


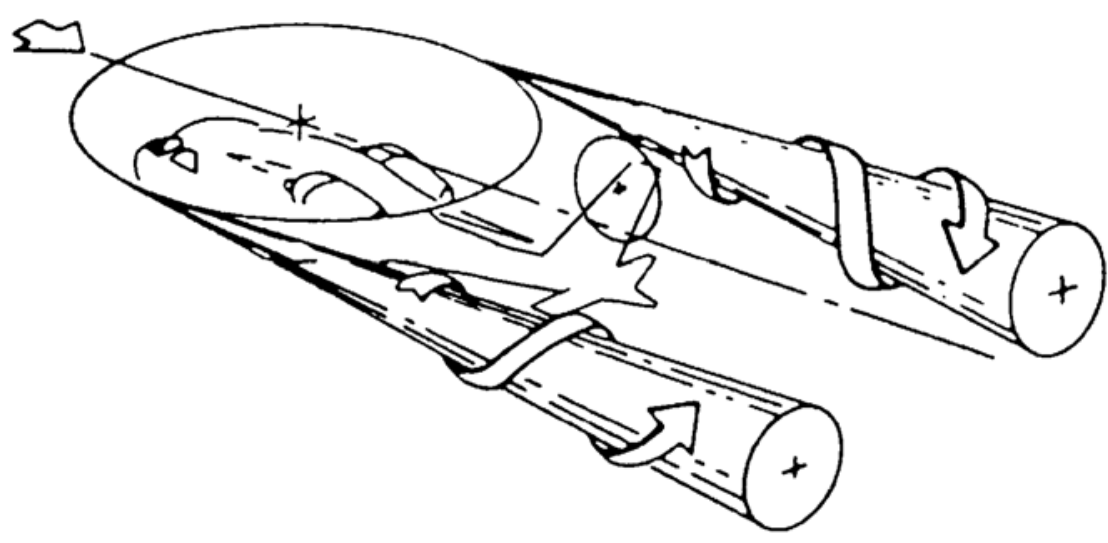

Figure 10 Illustration of the roll-up of helicopter tip vortices during forward flight 
(a) Swirl velocity

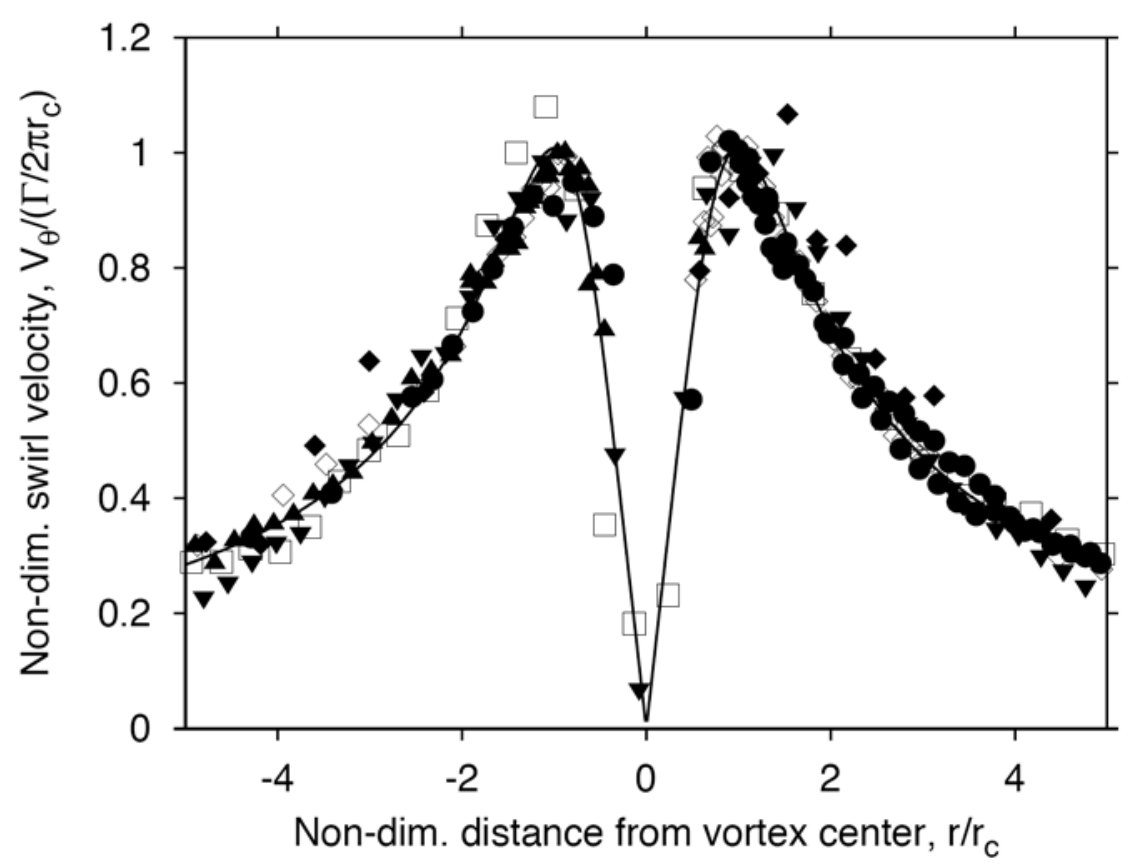

(b) Axial velocity

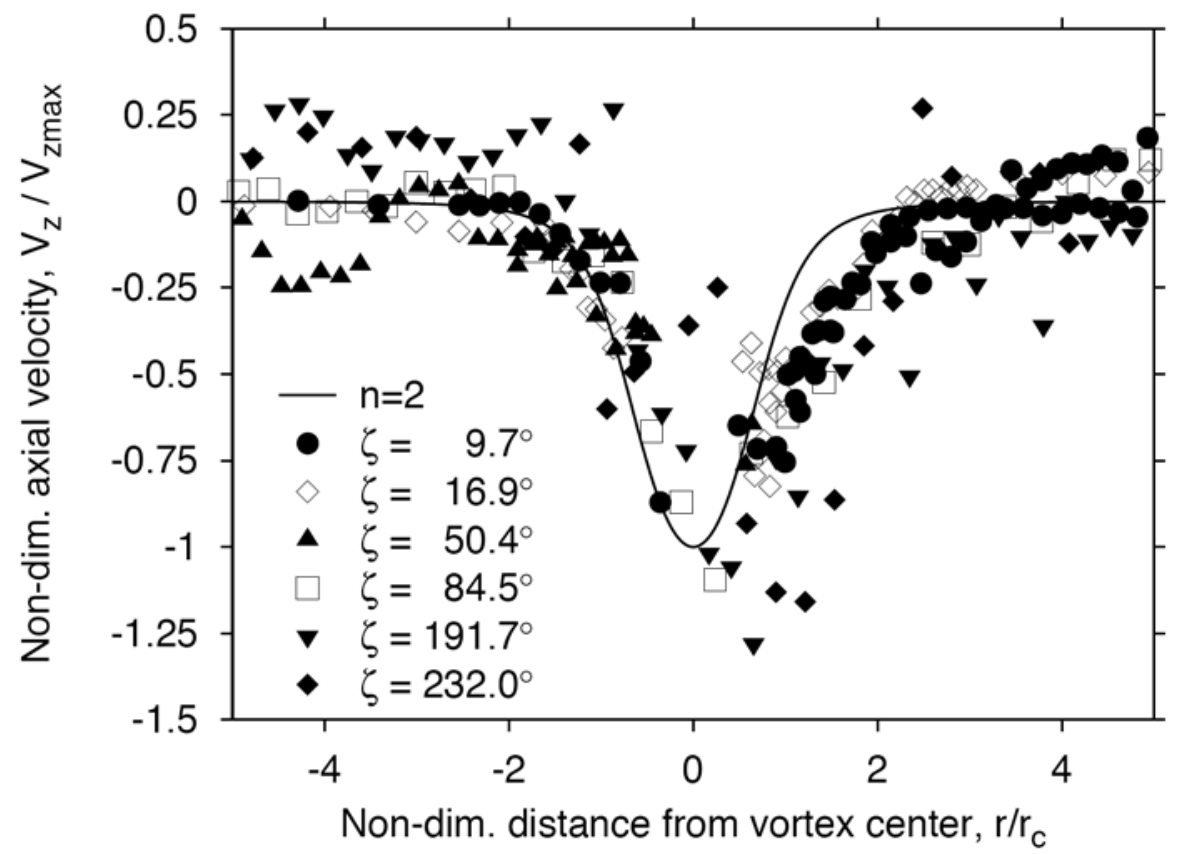

Figure 11. Measured axial and swirl velocities in the tip vortex of a model helicopter rotor compared with an analytical model prediction [34] 


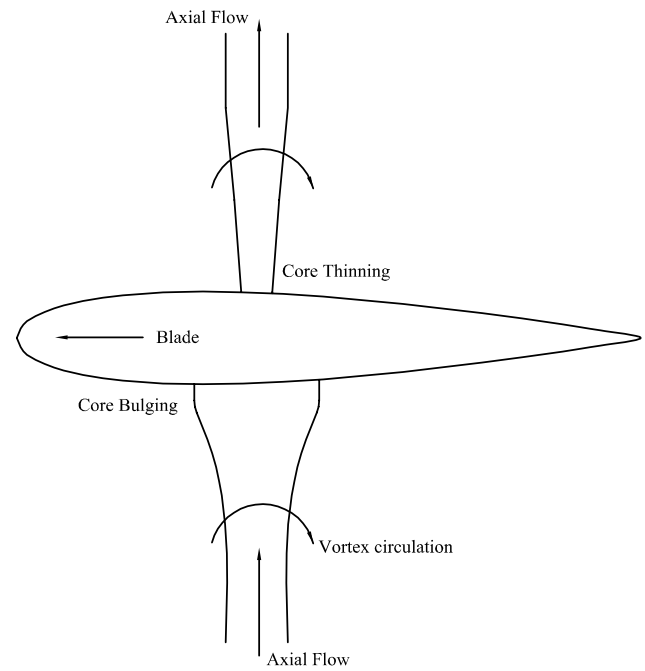

Figure 12 Simplistic illustration of vortex deformation during the OBVI cutting process 


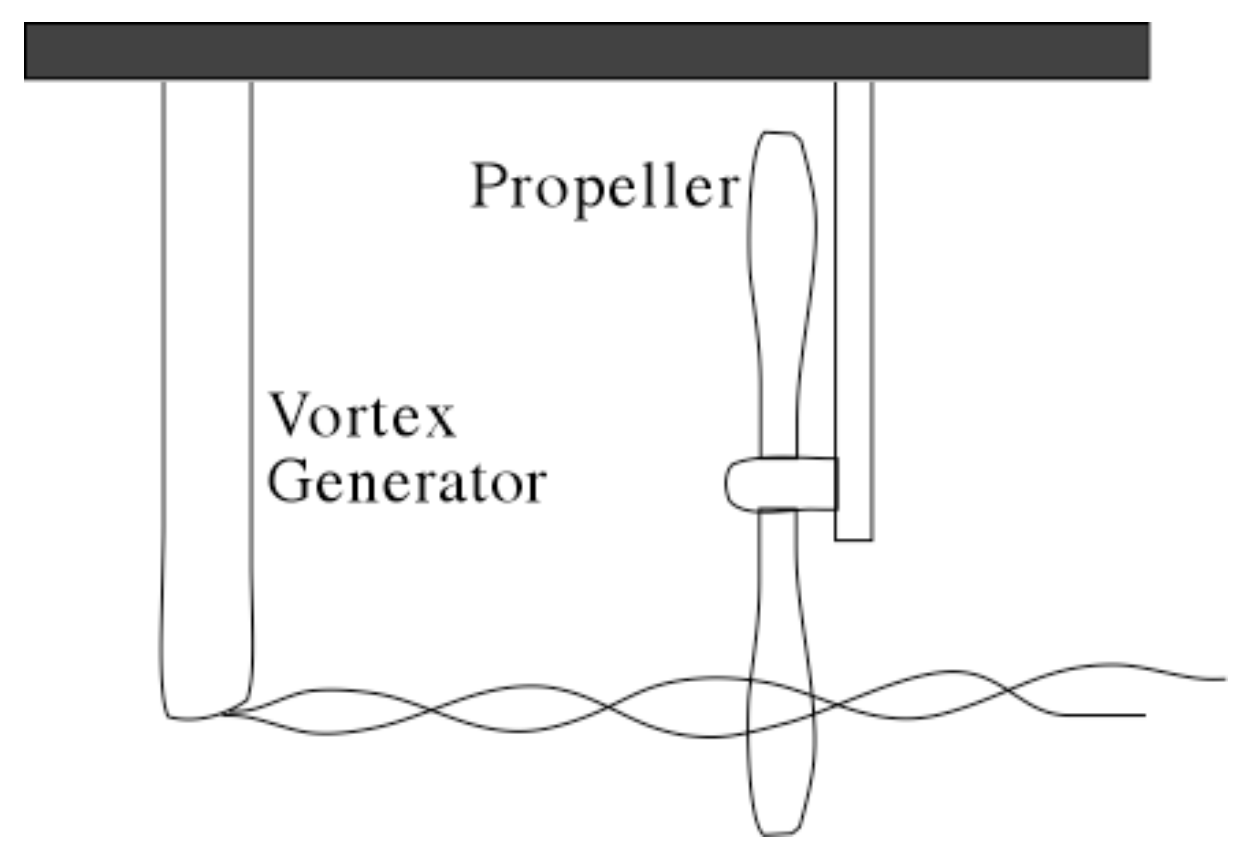

Figure 13. Schematic of the test set-up used by Shlinker and Amiet [45] during their experiments on tail rotor blade vortex interaction 


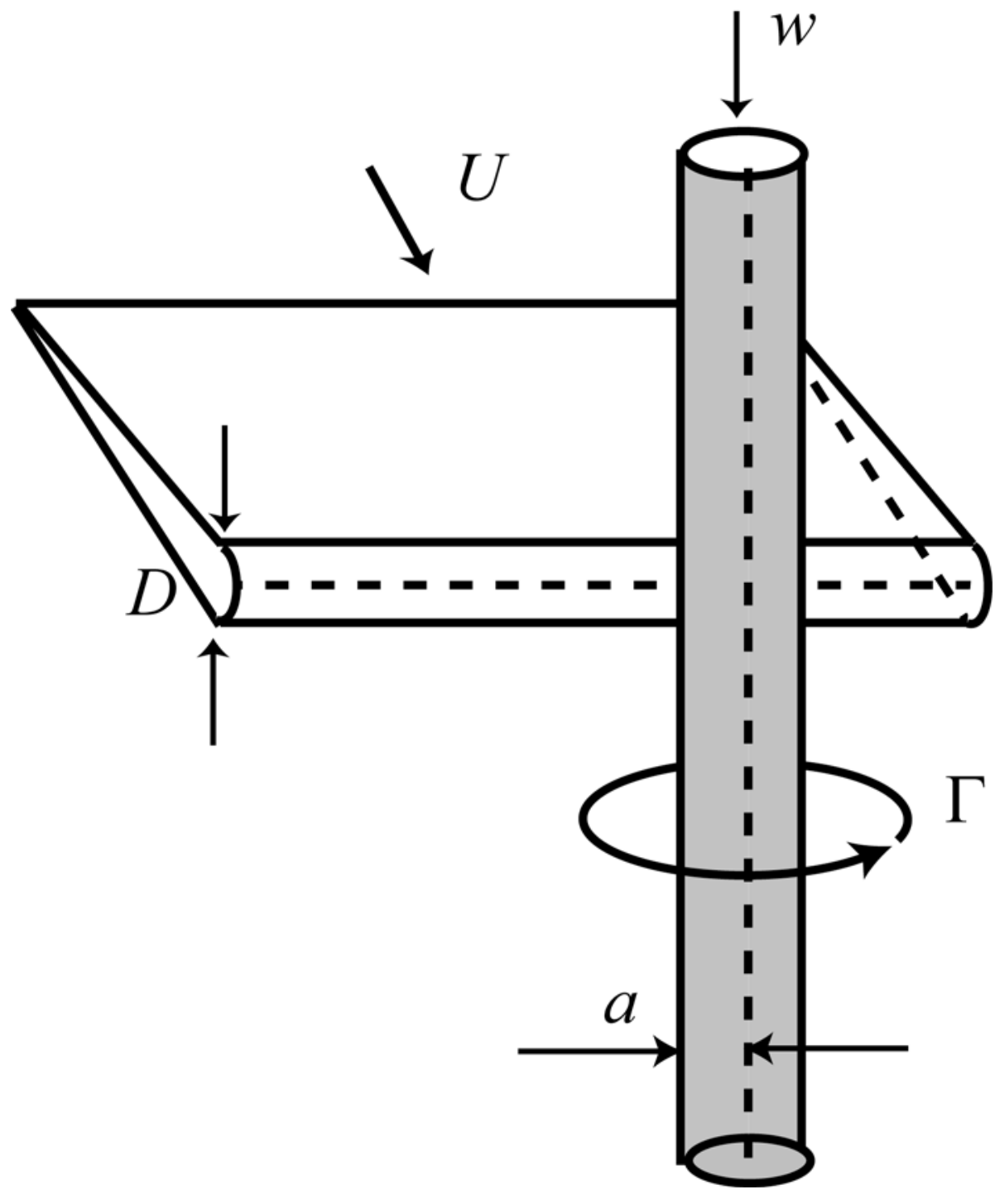

Figure 14. Schematic diagram showing parameters for a blade passing orthogonally into the vortex of a columnar vortex. 


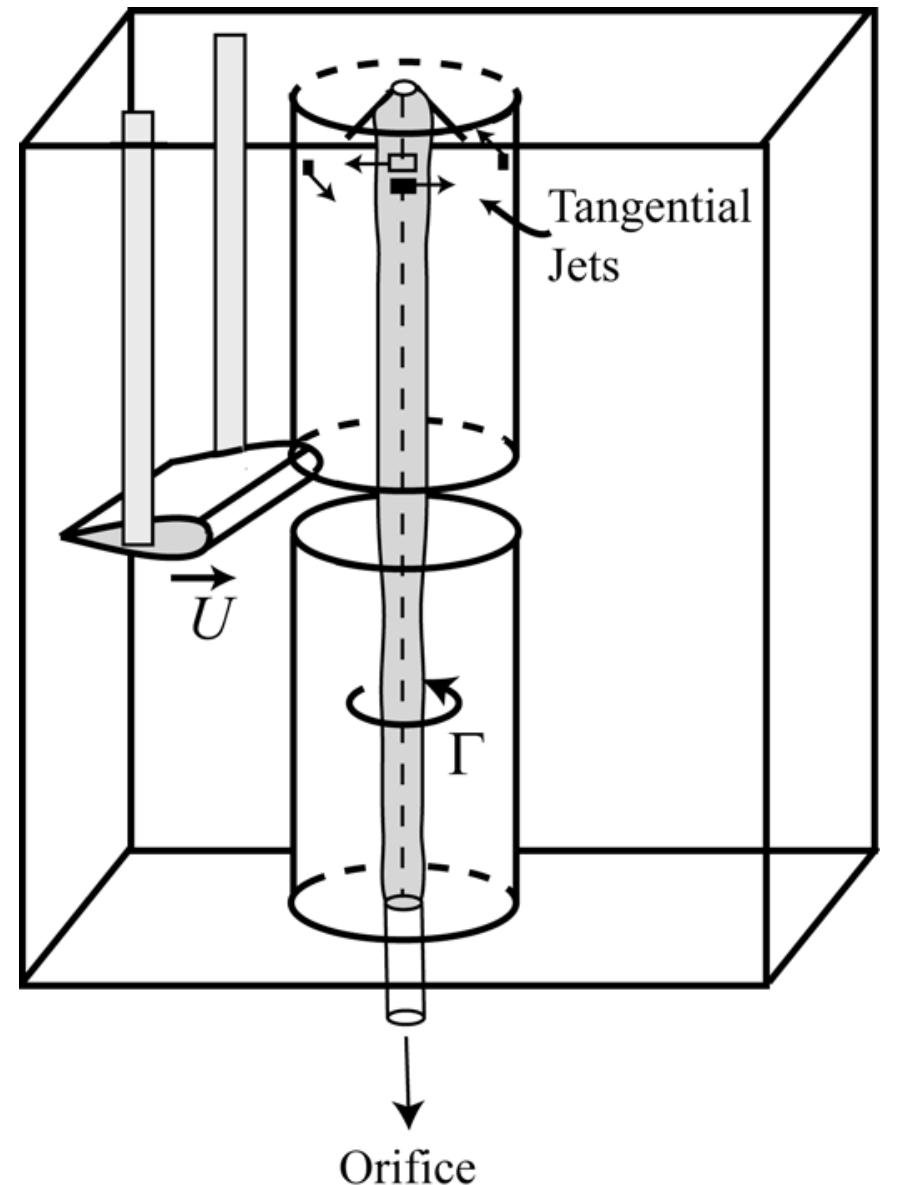

Figure 15. Schematic of the apparatus used for the University of Iowa experiments 


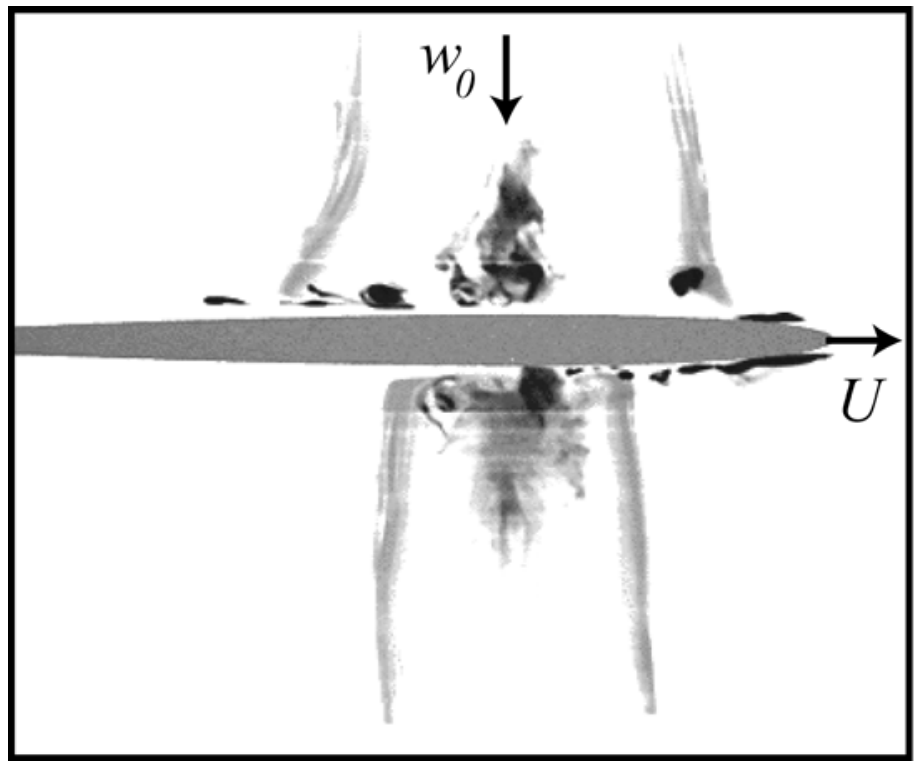

Figure 16. Planar LIF photograph showing the response of a vortex core with downward axial flow to cutting by a blade with impact parameter $I=0.3[50]$. 


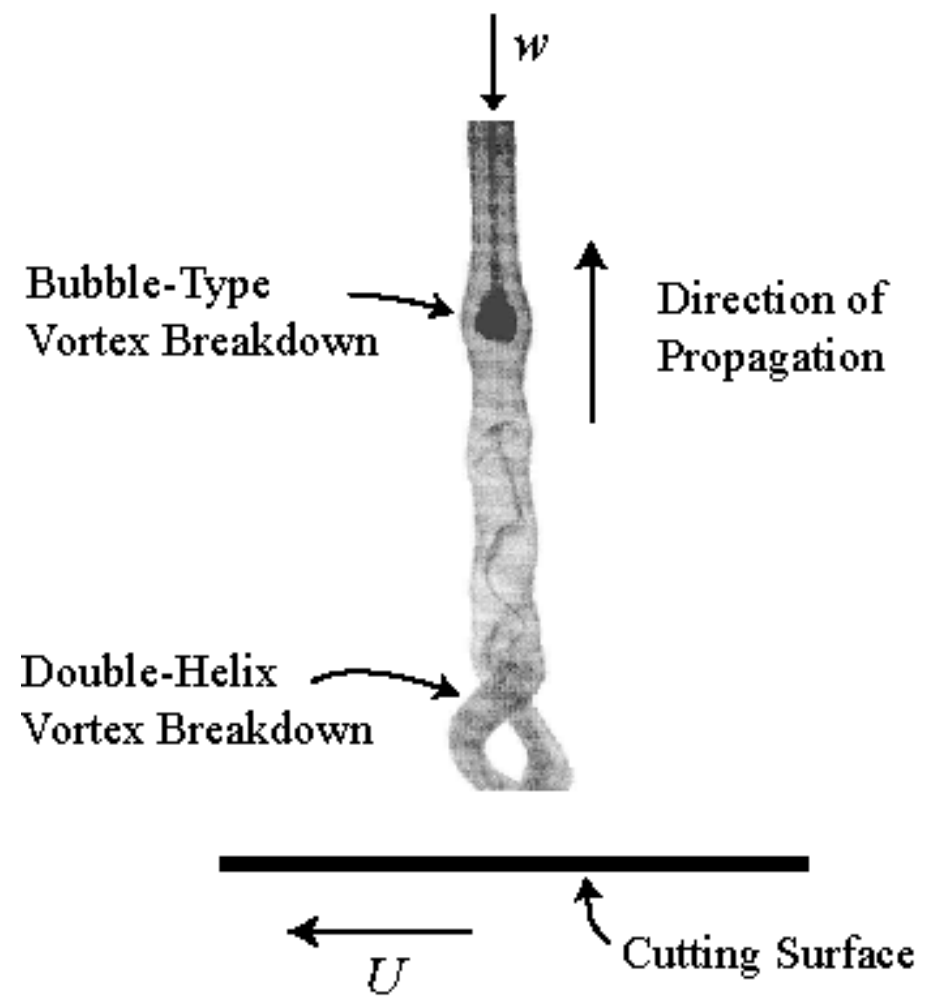

Figure 17. Upstream-propagating bubble-type vortex breakdown, followed by a helical breakdown, on the vortex core following cutting by a blade [49]. The cutting surface is shown schematically in the figure. 


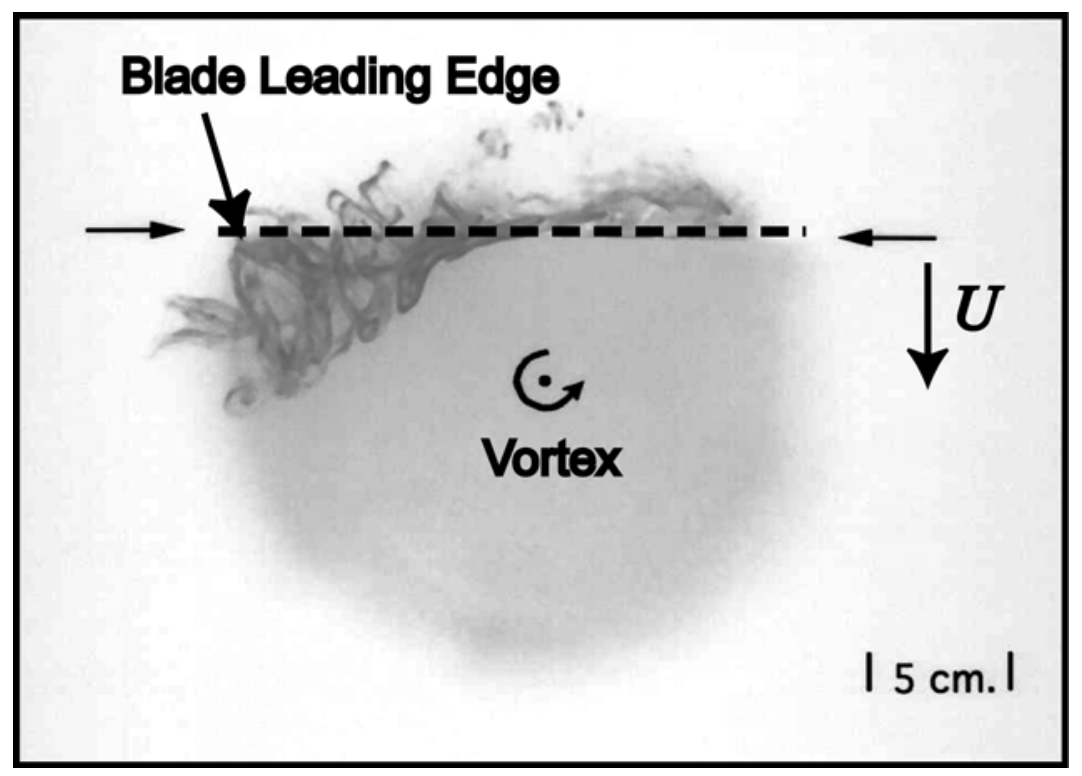

Figure 18. Planar LIF photograph in the plane of the blade showing ejection of boundary layer fluid from the blade leading edge for a case with $I=0.05[8]$. 


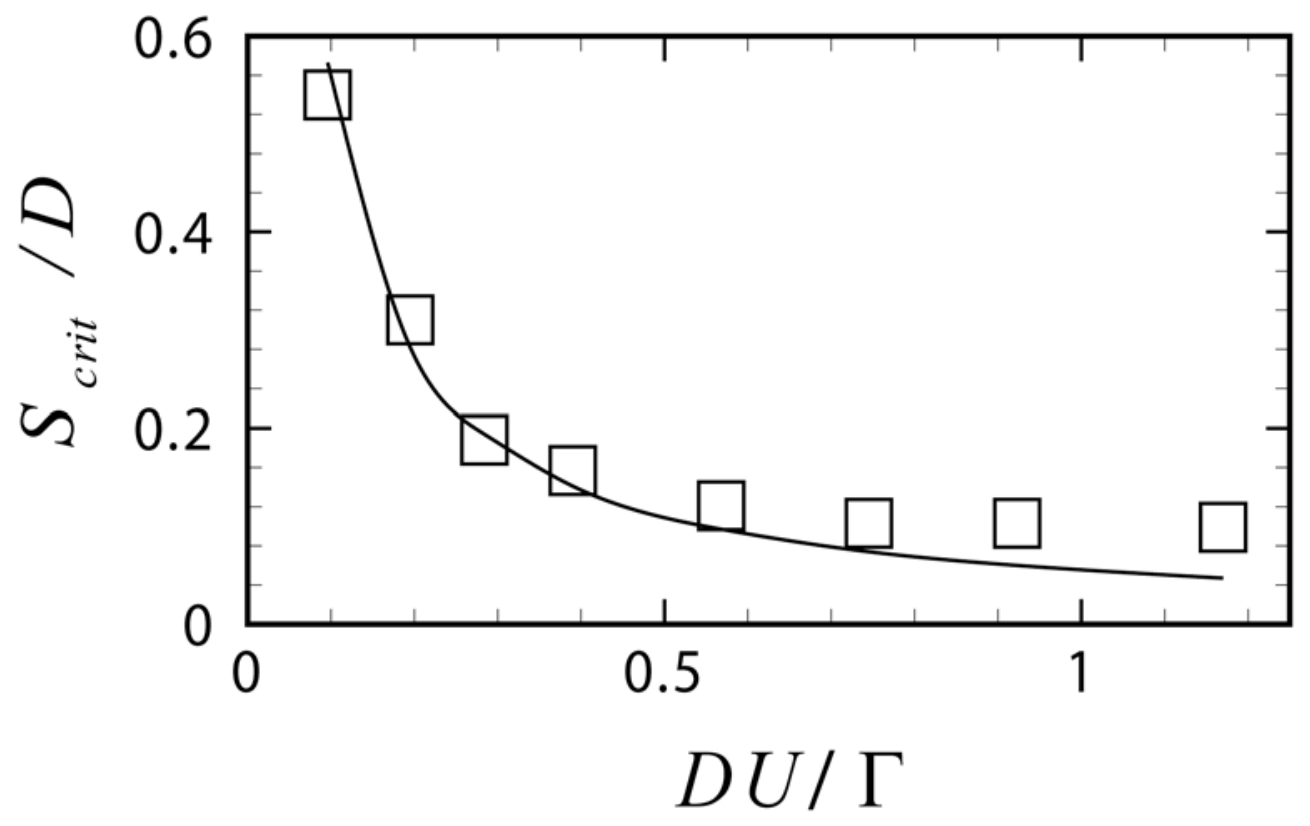

Figure 19. Plot showing experimental results (squares) and theoretical prediction (solid curve) for critical vortexbody separation distance $S_{\text {crit }}$ in vortex-cylinder interaction coinciding with onset of separation from the cylinder leading edge [54]. 


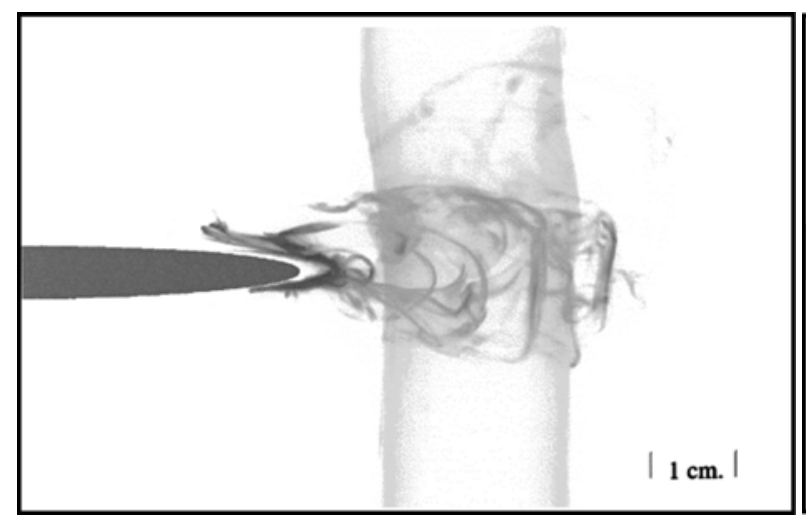

(a)

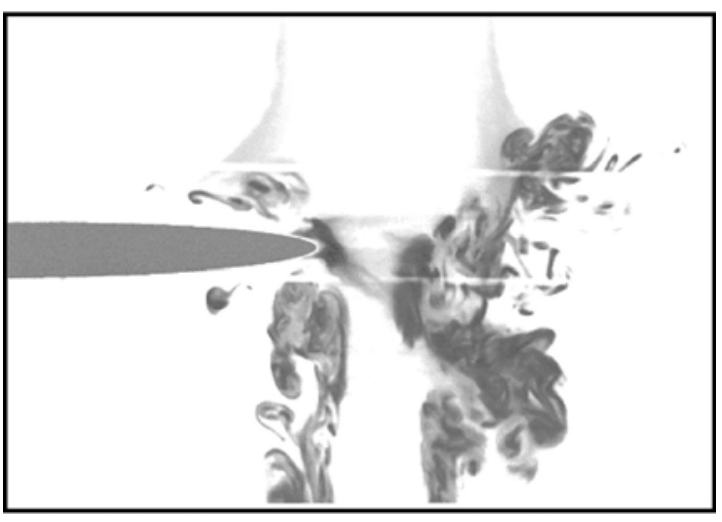

(b)

Figure 20 LIF photographs of blade-vortex interaction with $I=0.05$ showing (a) three-dimensional view of vorticity loops formed from vorticity ejected from blade boundary layer and (b) planar view of turbulence wrapping around the columnar vortex after long time [8]. 


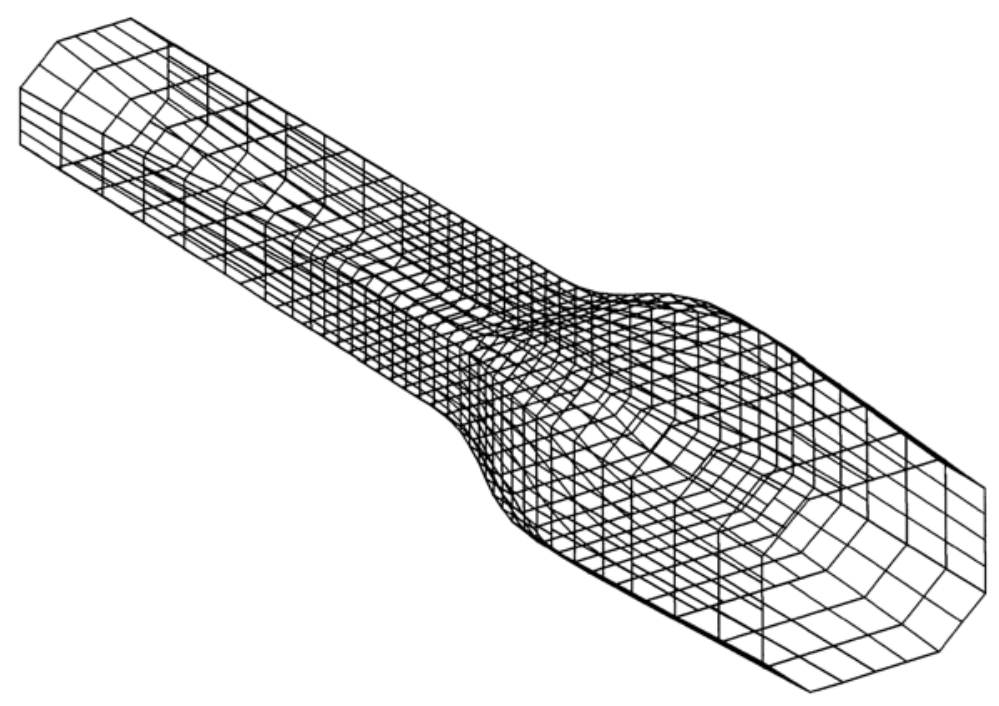

Figure 21. Source panel distribution representing the Glasgow University $1.15 \mathrm{~m} \times 0.85 \mathrm{~m}$ low speed wind tunnel contraction, working section and high-speed diffuser [57]. 


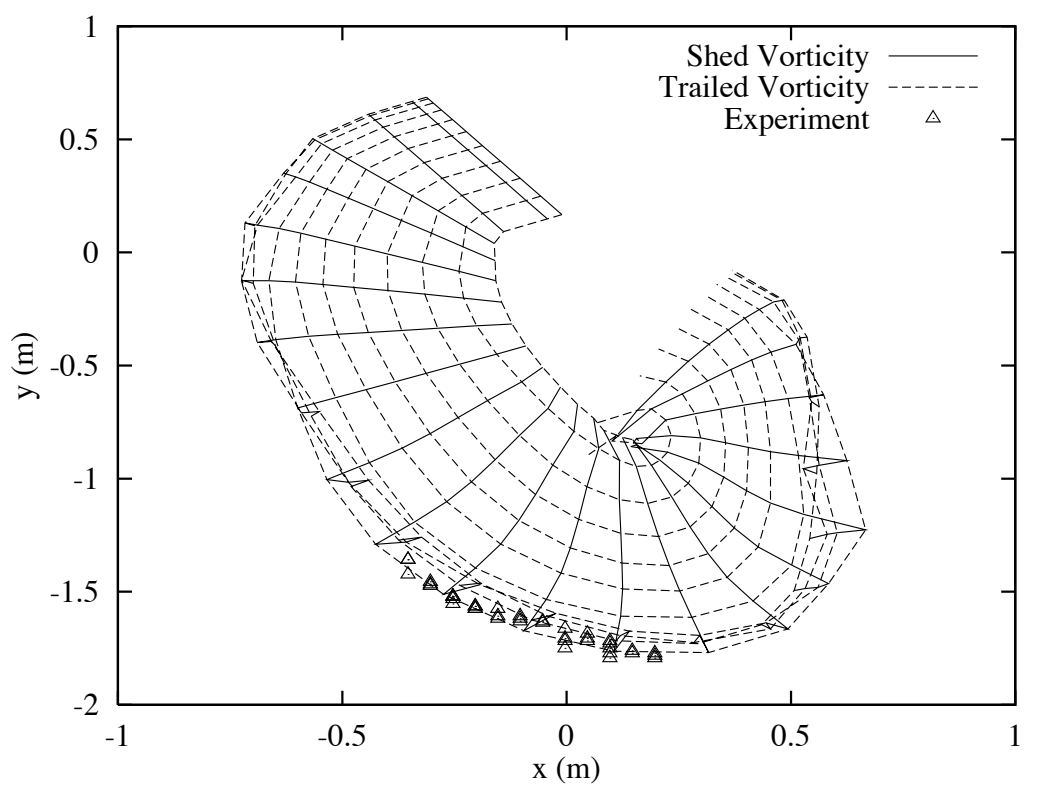

Figure 22 Comparison of computed wake geometry with hot-wire measurements of the wake produced by the single-bladed vortex generator in the Glasgow University $1.15 \mathrm{~m} \times 0.85 \mathrm{~m}$ low speed wind tunnel [60]. 


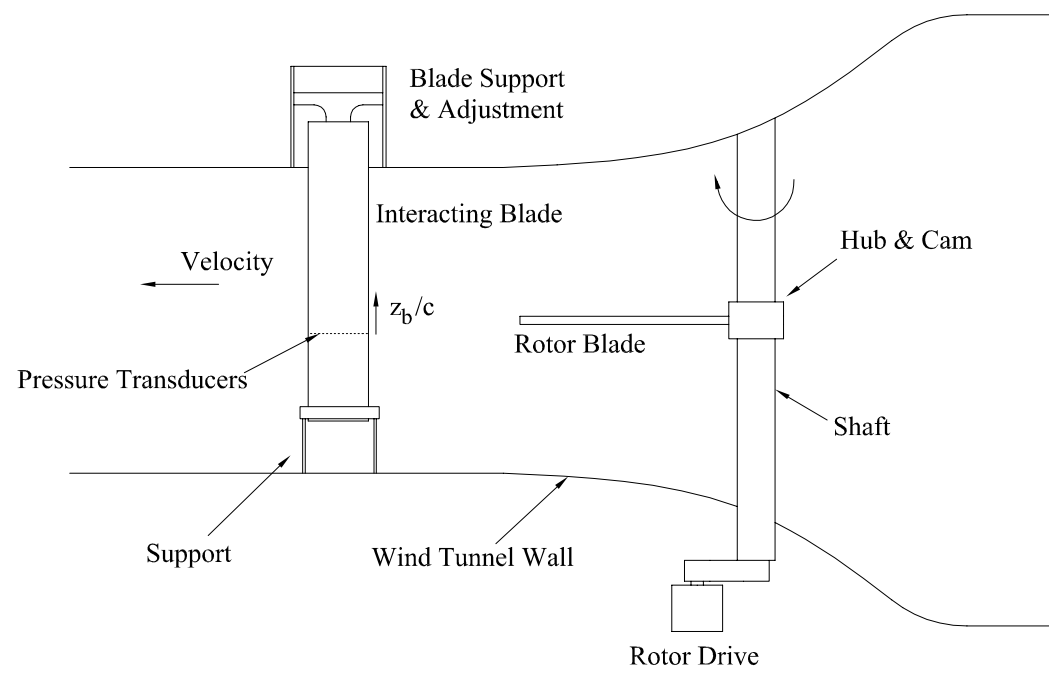

Figure 23 Test set-up in Glasgow University $1.15 \mathrm{~m}$ x $0.85 \mathrm{~m}$ low speed wind tunnel 


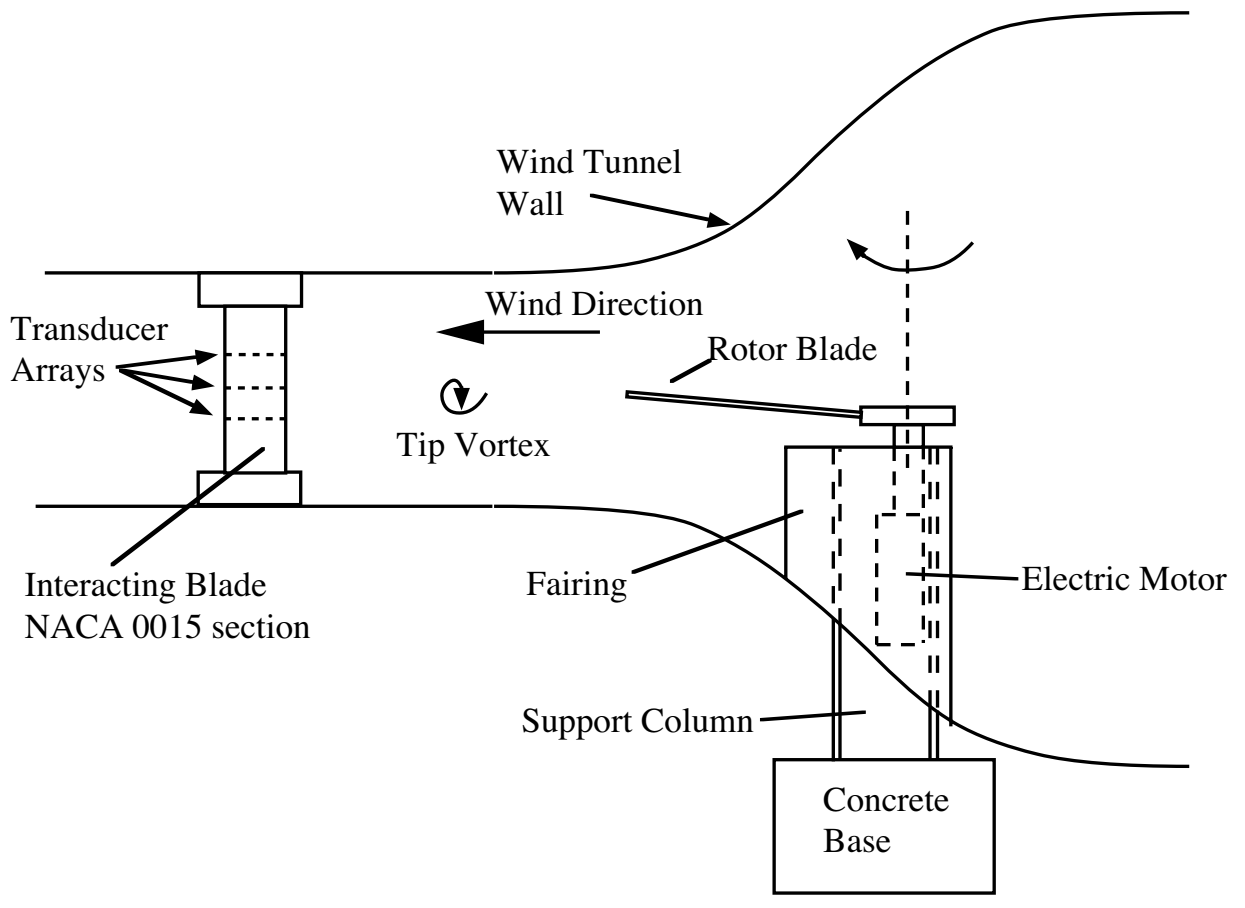

Figure 24 Test Set-up in the Glasgow University $2.65 \mathrm{~m}$ x 2.04m 'Argyll' low speed wind tunnel 


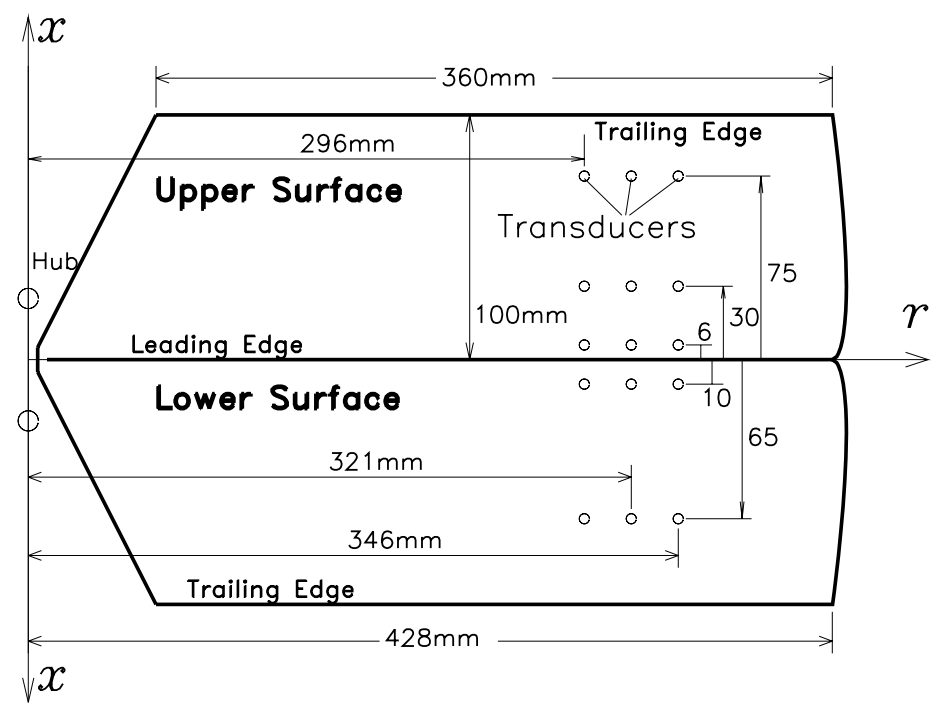

Figure 25 Pressure transducer layout on model tail rotor blade used in testing in the Glasgow University $2.65 \mathrm{~m} \mathrm{x}$ $2.04 \mathrm{~m}$ low speed wind tunnel 


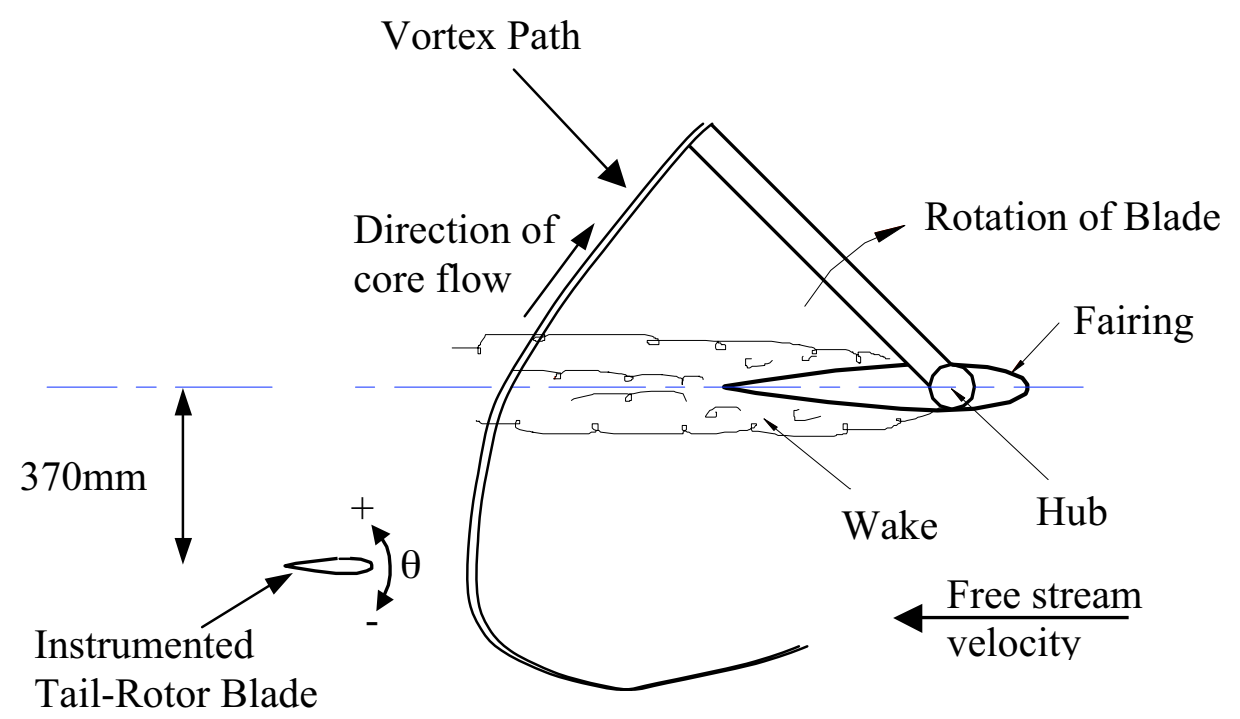

Figure 26 Plan view of test set-up in the Glasgow University $2.65 \mathrm{~m} \times 2.04 \mathrm{~m}$ low speed wind tunnel showing lateral position of tail rotor 


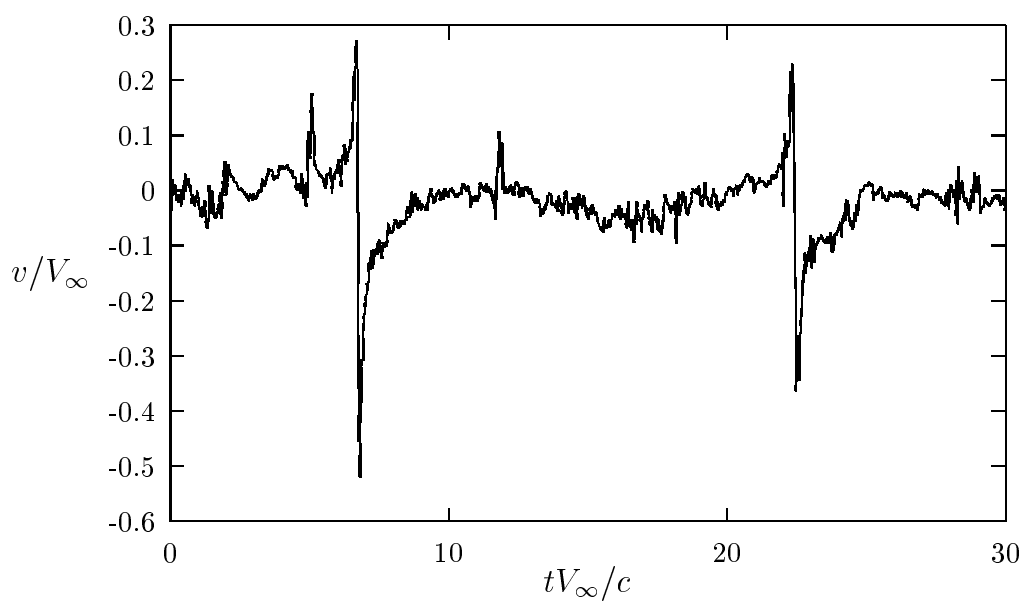

Figure 27 Typical vertical velocity trace measured through the core of the tip vortex produced by the vortex generator in the Glasgow University $1.15 \mathrm{~m} \times 0.85 \mathrm{~m}$ low speed wind tunnel 


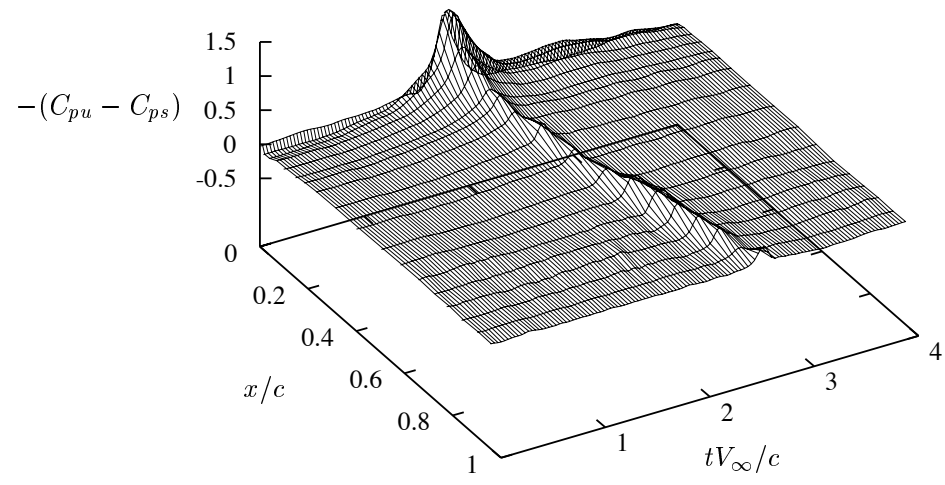

Figure 28a Unsteady upper surface pressures during a clean orthogonal interaction measured in the Glasgow University $1.15 \mathrm{~m} \times 0.85 \mathrm{~m}$ low speed wind tunnel [61]

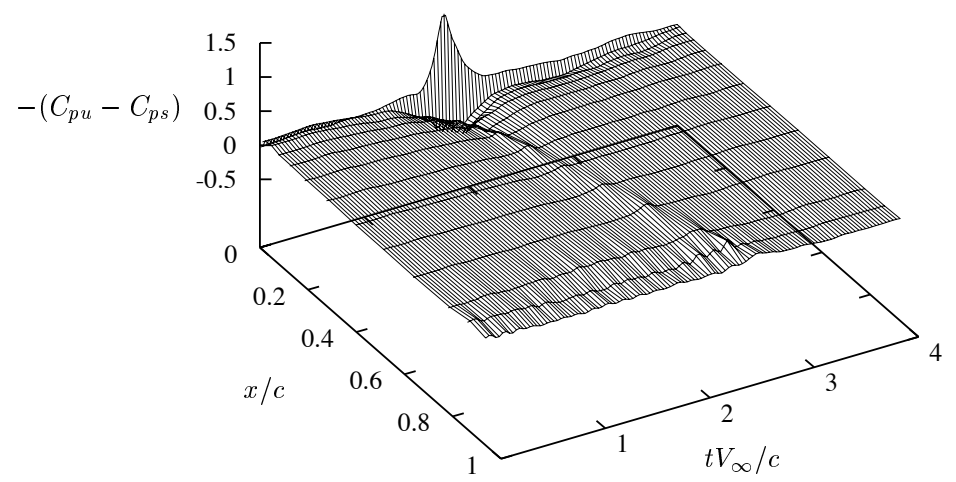

Figure 28b Unsteady lower surface pressures during a clean orthogonal interaction measured in the Glasgow University $1.15 \mathrm{~m} \times 0.85 \mathrm{~m}$ low speed wind tunnel [61] 


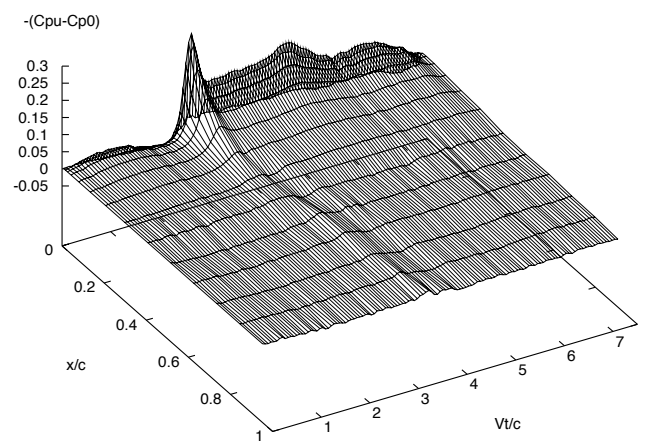

Figure 29a Unsteady upper surface pressure distribution measured in the Glasgow University $2.65 \mathrm{~m} \times 2.04 \mathrm{~m}$ low speed wind tunnel [63]

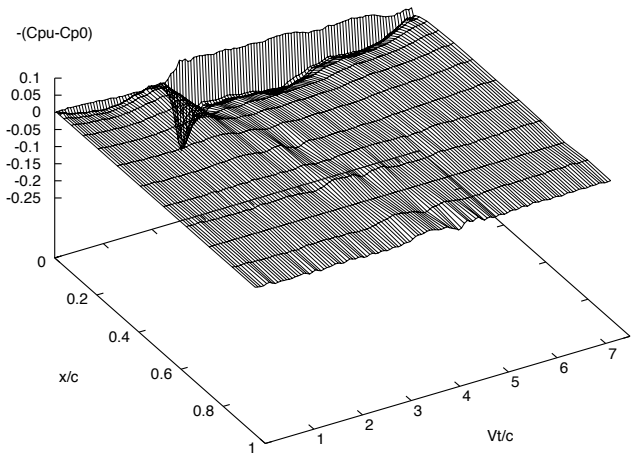

Figure 29b Unsteady lower surface pressure distribution measured in the Glasgow University $2.65 \mathrm{~m} \times 2.04 \mathrm{~m}$ low speed wind tunnel [63] 


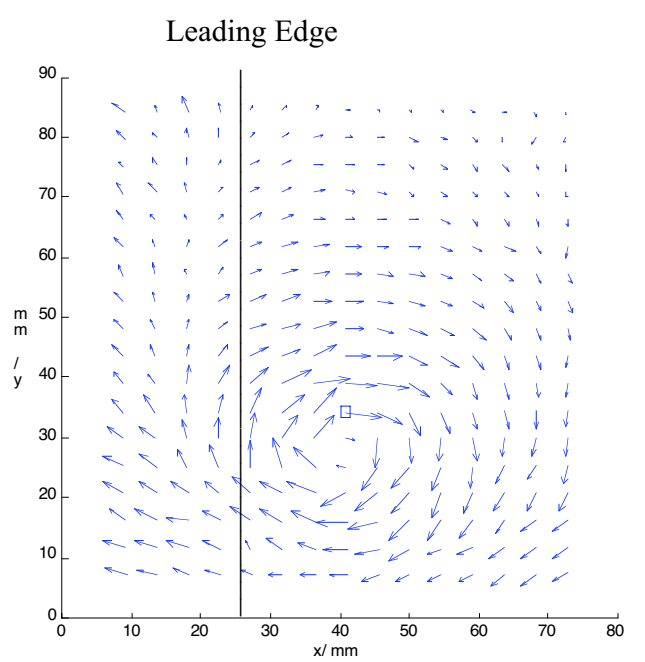

(a) Vortex approaching leading edge

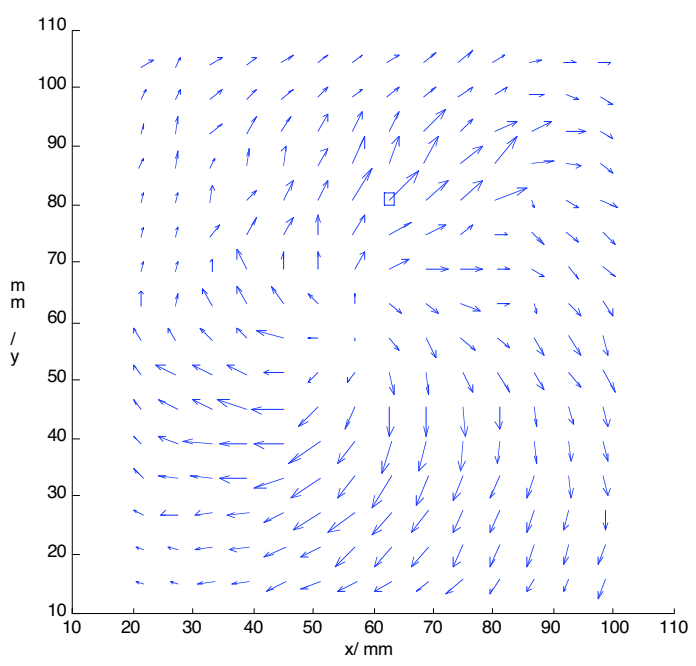

(c) Vortex just past mid-chord

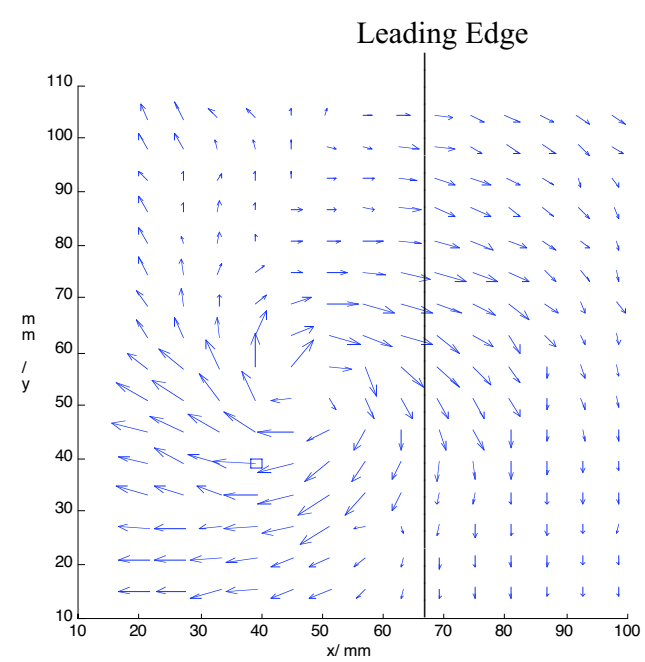

(b) Vortex crossing leading edge

Figure 30. PIV measurement of OBVI on the lower surface of a blade. The mean flow, from left to right, has been removed for clarity. 


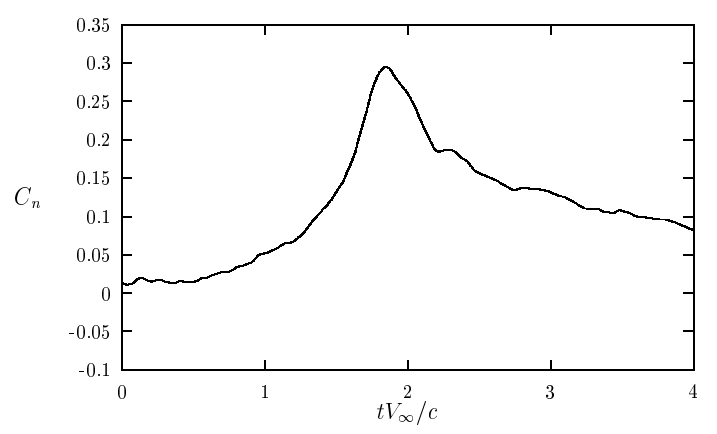

Figure 31a Normal force coefficient during clean orthogonal interaction measured in the Glasgow University $1.15 \mathrm{~m}$ $\mathrm{x} 0.85 \mathrm{~m}$ low speed wind tunnel [61]

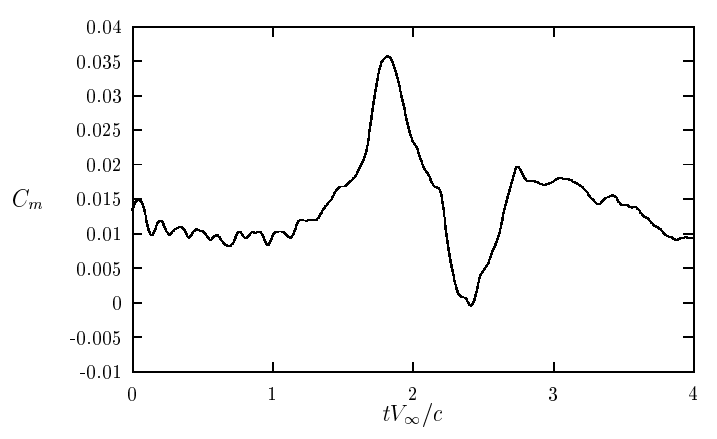

Figure $31 \mathrm{~b}$ Pitching moment coefficient during clean orthogonal interaction measured in the Glasgow University $1.15 \mathrm{~m} \times 0.85 \mathrm{~m}$ low speed wind tunnel [61] 


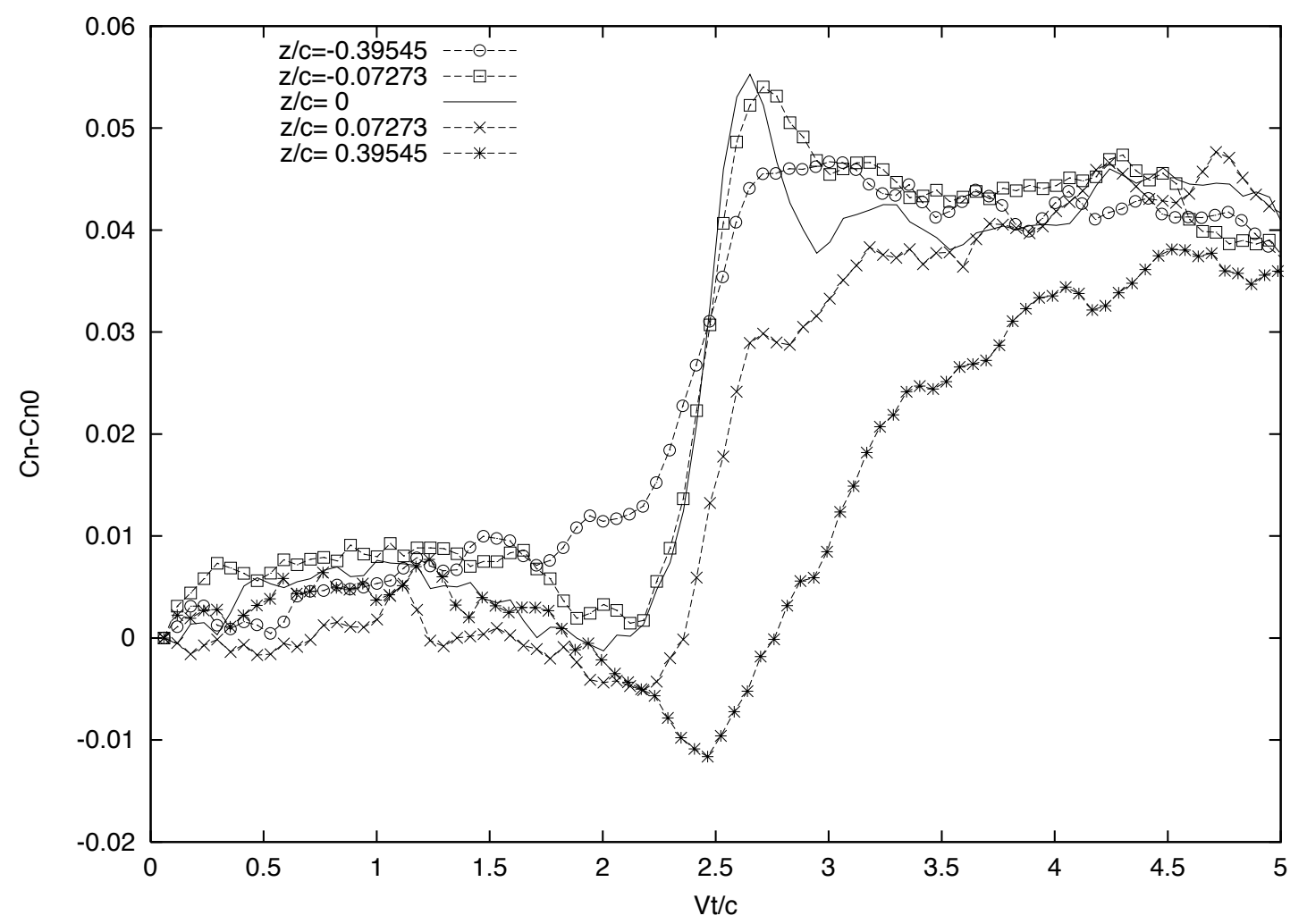

Figure 32 Normal force coefficient response at a series of positions relative to the vortex core passage height measured in the Glasgow University $2.65 \mathrm{~m} \times 2.04 \mathrm{~m}$ low speed wind tunnel [63] 


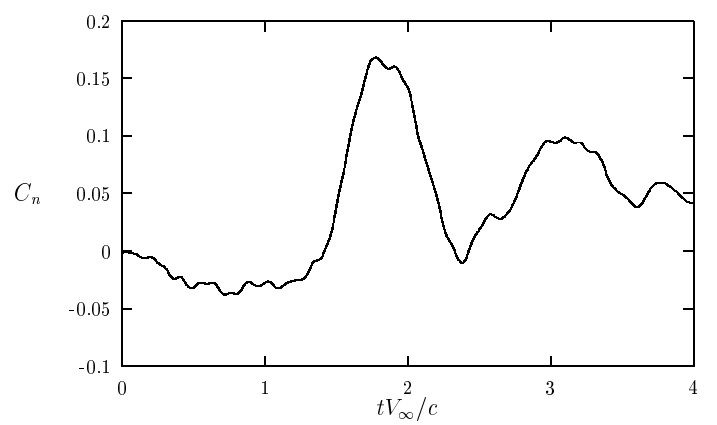

Figure 33a Normal force coefficient during non- orthogonal interaction measured in the Glasgow University $1.15 \mathrm{~m} x$ $0.85 \mathrm{~m}$ low speed wind tunnel [61]

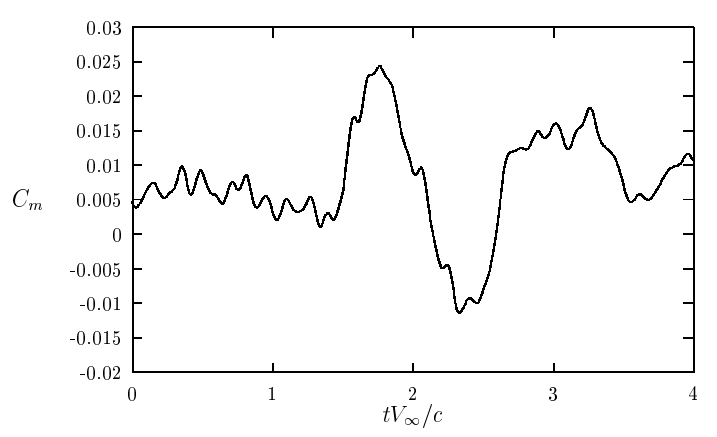

Figure 33b Pitching moment coefficient during non- orthogonal interaction measured in the Glasgow University $1.15 \mathrm{~m} \times 0.85 \mathrm{~m}$ low speed wind tunnel [61] 


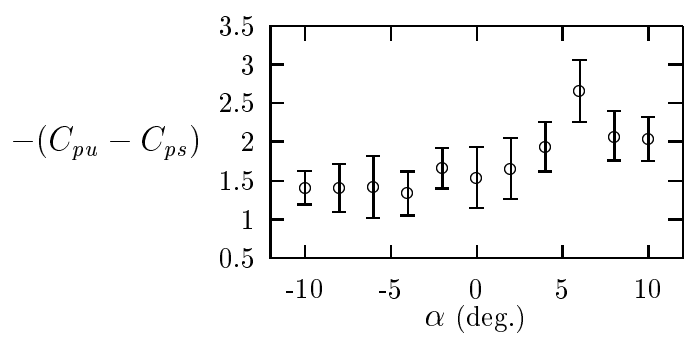

Figure 34a Variation of upper surface leading edge peak suction versus incidence during orthogonal interaction measured in the Glasgow University $1.15 \mathrm{~m}$ x $0.85 \mathrm{~m}$ low speed wind tunnel [36]

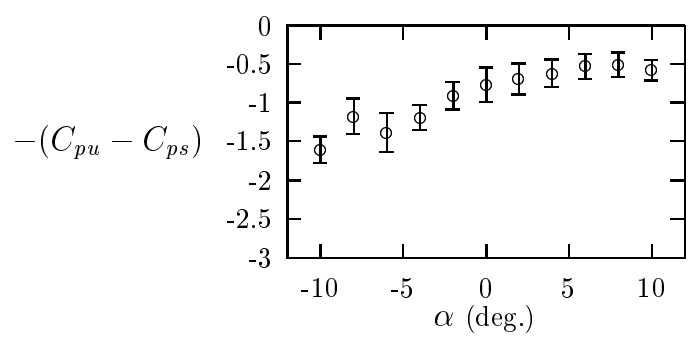

Figure $34 \mathrm{~b}$ Variation of lower surface leading edge peak suction versus incidence during orthogonal interaction measured in the Glasgow University $1.15 \mathrm{~m} \times 0.85 \mathrm{~m}$ low speed wind tunnel [36] 


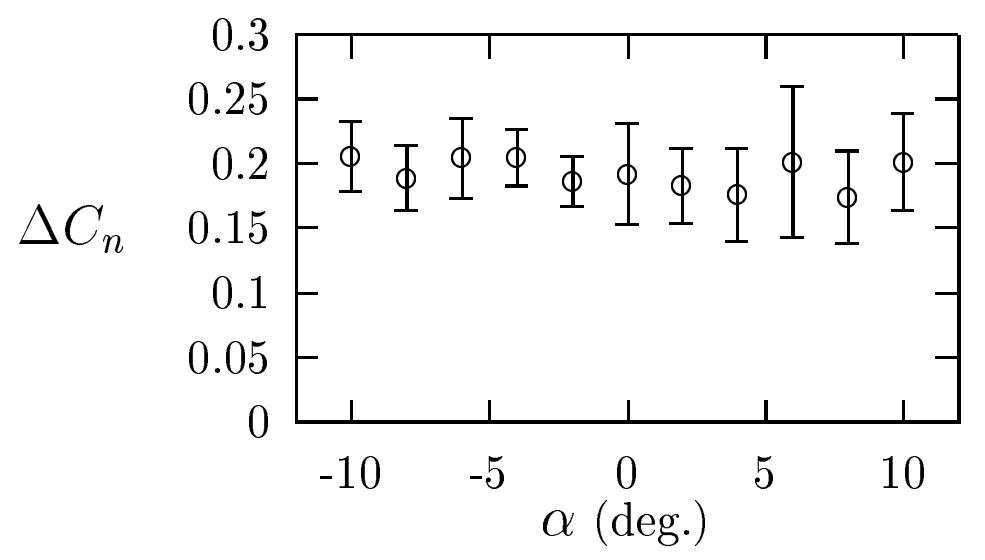

Figure 35 Variation of impulsive normal force with incidence during OBVI measured in the Glasgow University $1.15 \mathrm{~m} \times 0.85 \mathrm{~m}$ low speed wind tunnel [36] 


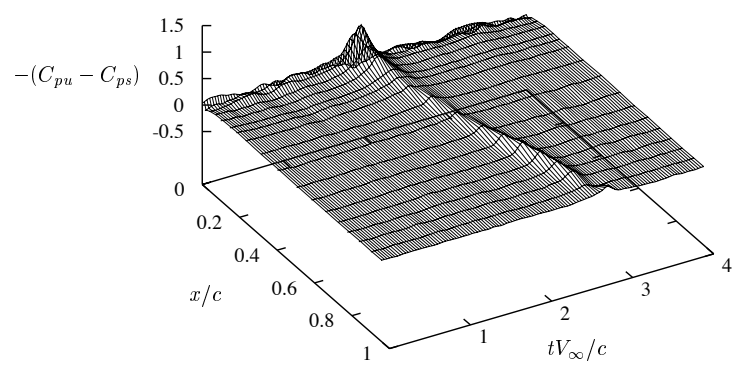

Figure 36a Unsteady upper surface pressures during a secondary orthogonal interaction measured in the Glasgow University $1.15 \mathrm{~m} \mathrm{x} 0.85 \mathrm{~m}$ low speed wind tunnel [37]

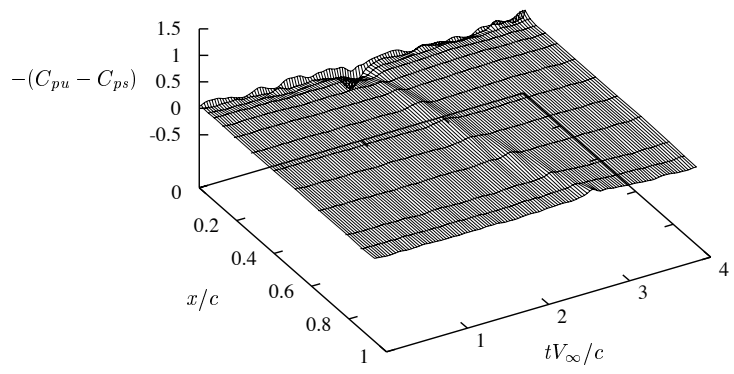

Figure $36 \mathrm{~b}$ Unsteady lower surface pressures during a secondary orthogonal interaction measured in the Glasgow University $1.15 \mathrm{~m} \times 0.85 \mathrm{~m}$ low speed wind tunnel [37] 


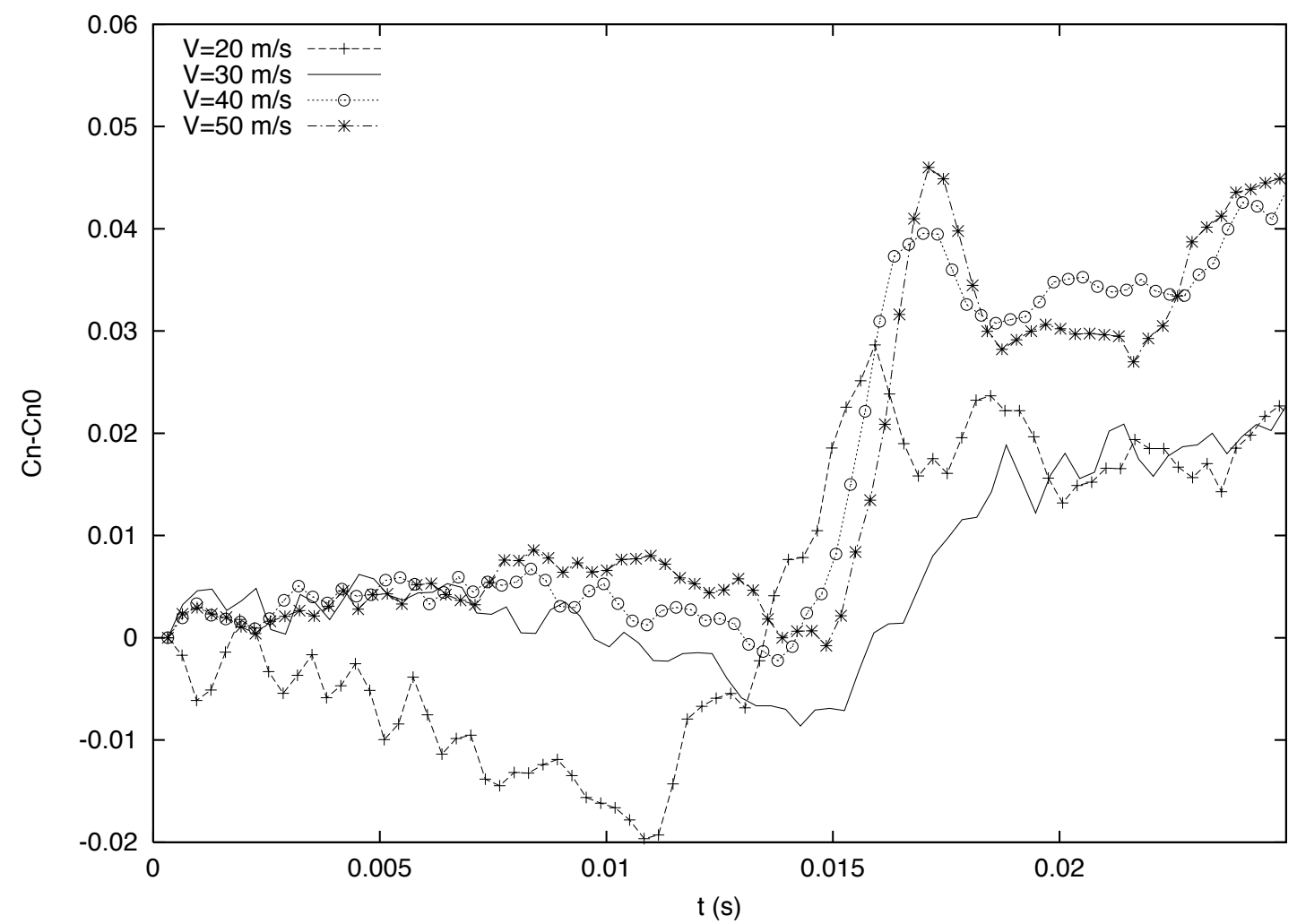

Figure 37. Variation of normal force coefficient during orthogonal interaction at $\alpha=6^{\circ}$ for different wind speeds measured in the Glasgow University $2.65 \mathrm{~m} \times 2.04 \mathrm{~m}$ low speed wind tunnel [63] 


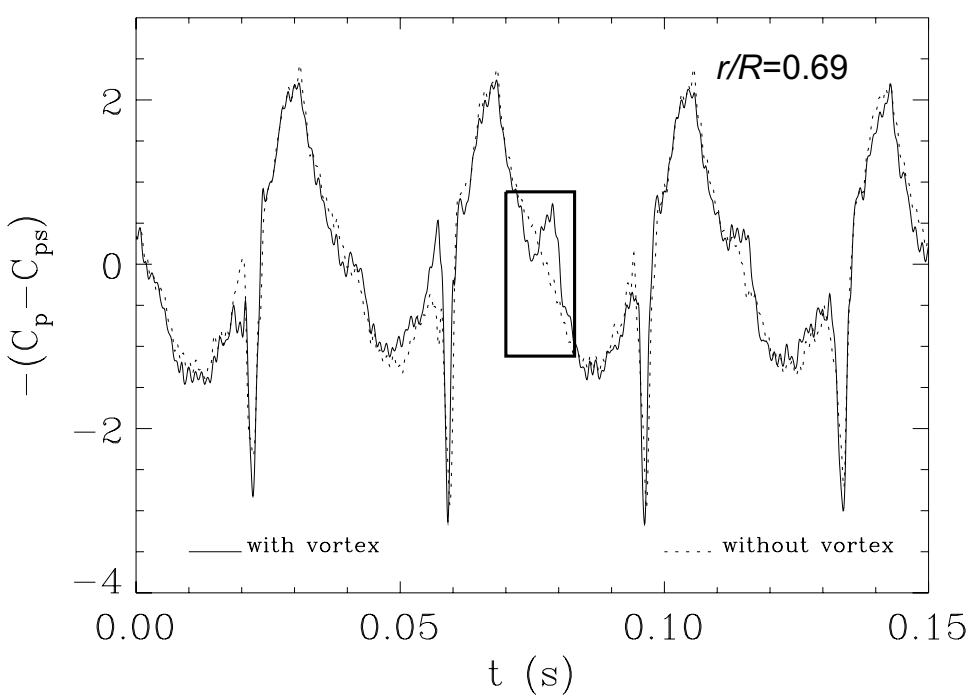

Figure 38 Pressure coefficient variations measured near the upper surface leading edge of a model tail rotor $(\mathrm{x} / \mathrm{c}=0.06, \mathrm{r} / \mathrm{R}=0.69)$ in the Glasgow University $2.65 \mathrm{~m} \times 2.04 \mathrm{~m}$ low speed wind tunnel [64]. The box highlights an OBVI event. 


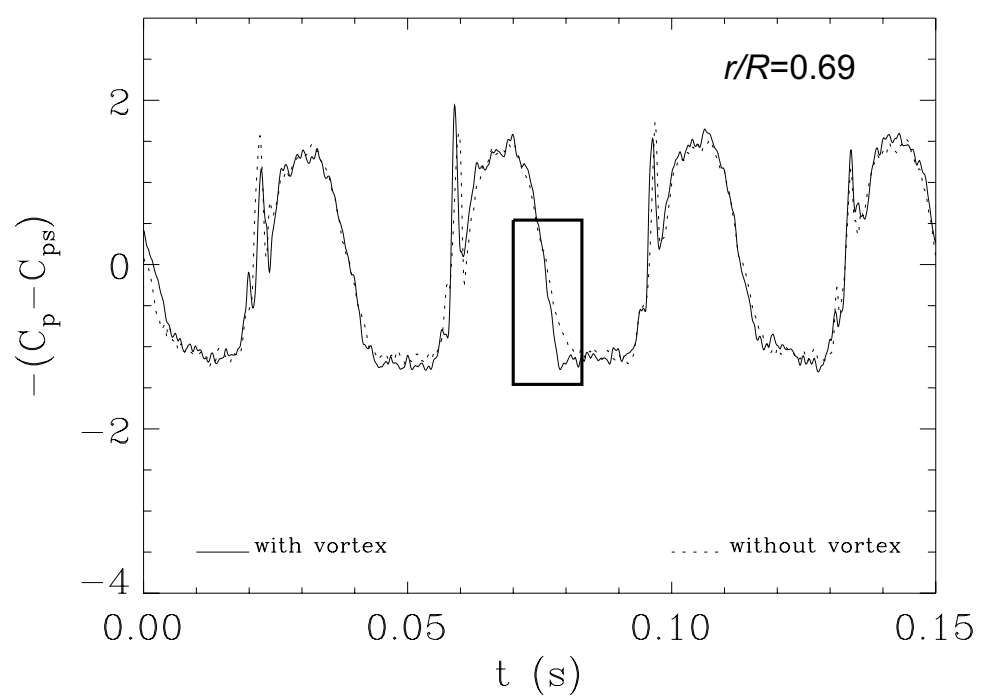

Figure 39 Pressure coefficient variations measured near the lower surface leading edge of a model tail rotor $(\mathrm{x} / \mathrm{c}=0.1$, $\mathrm{r} / \mathrm{R}=0.69$ ) in the Glasgow University $2.65 \mathrm{~m} \times 2.04 \mathrm{~m}$ low speed wind tunnel [64]. The box highlights an OBVI event. 

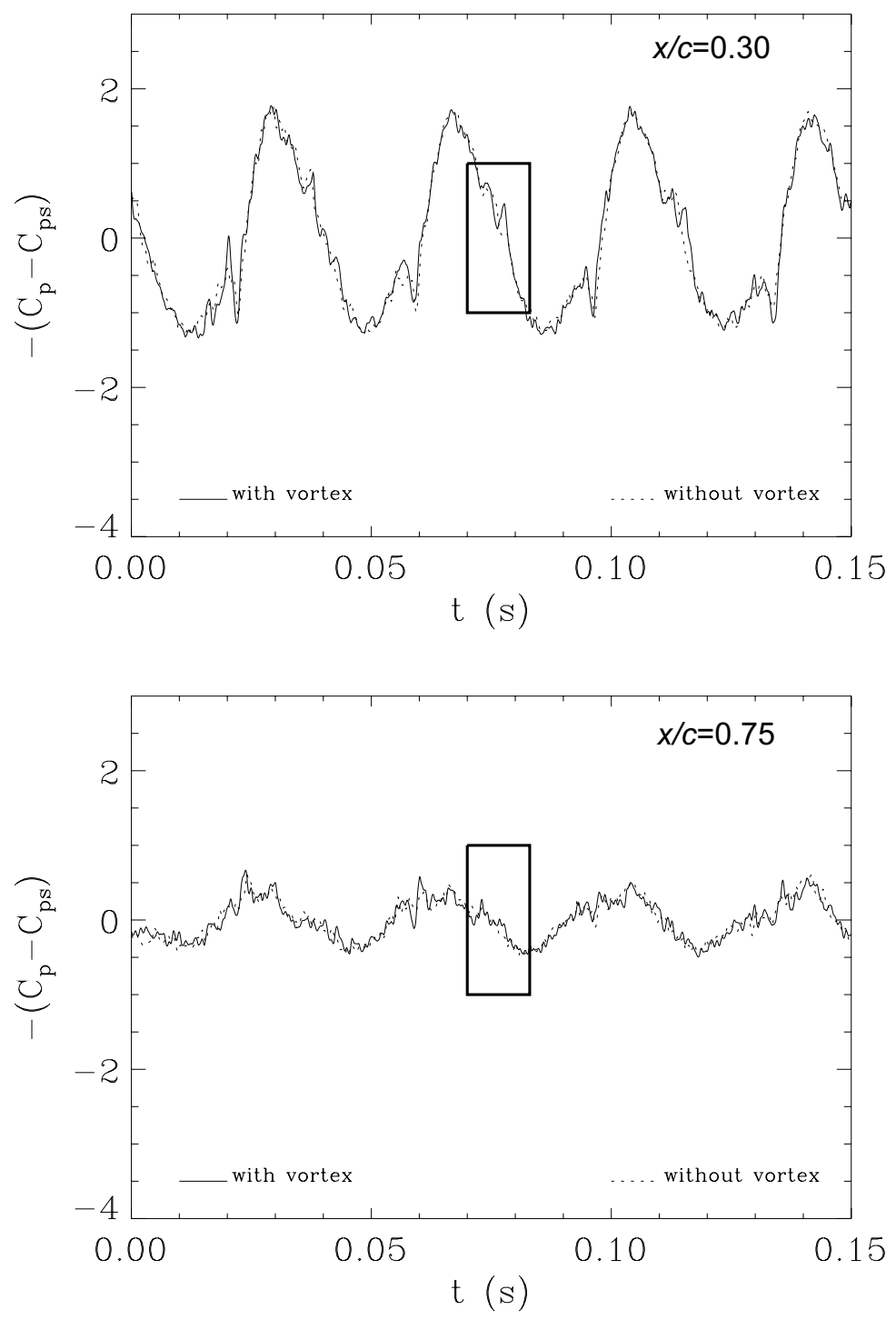

Figure 40 Pressure coefficient variations at two upper surface locations on a tail rotor blade undergoing blade vortex interaction measured in the Glasgow University $2.65 \mathrm{~m}$ x 2.04m low speed wind tunnel [64]. 


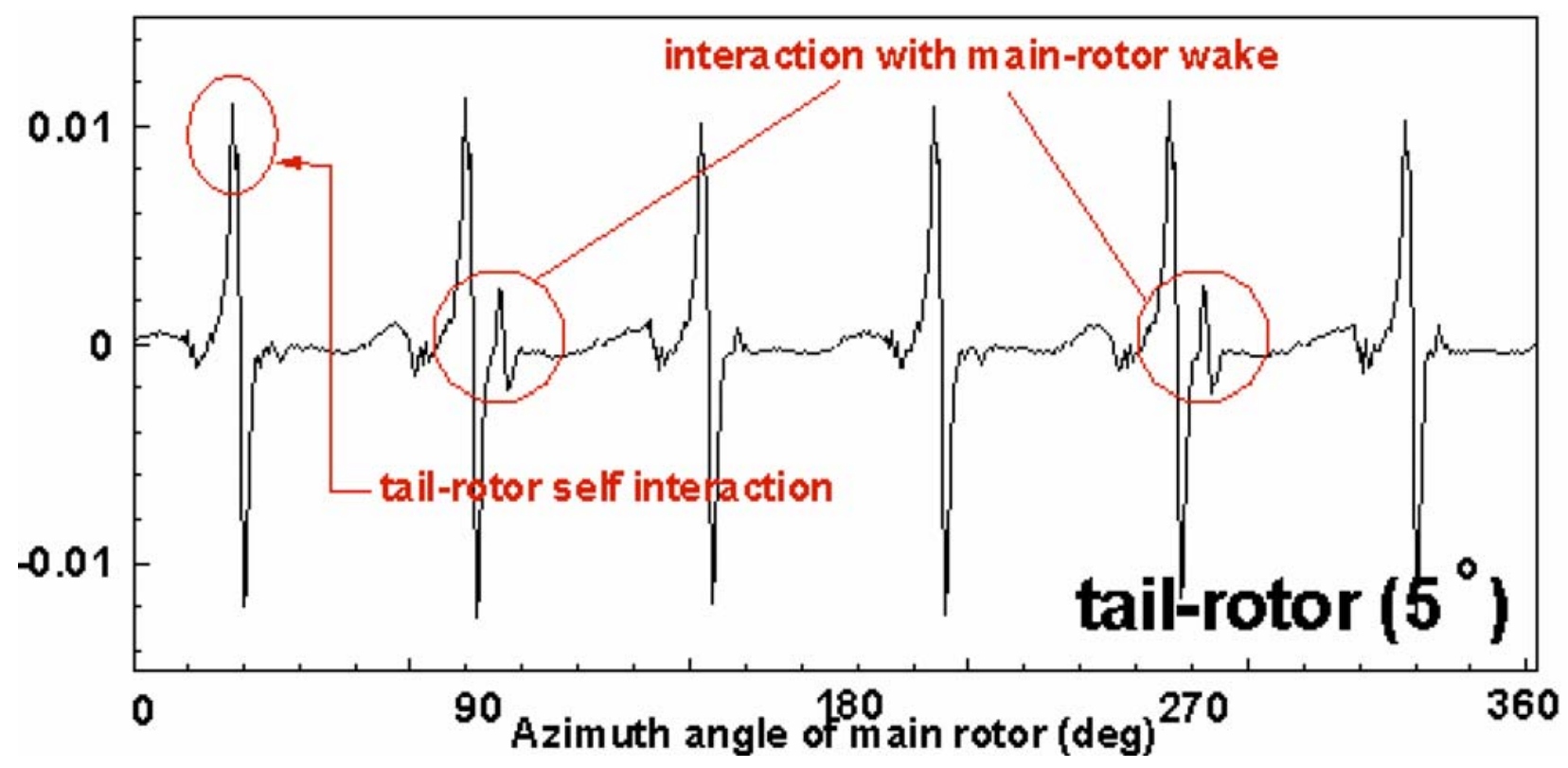

Figure 41 Computed tail rotor sound pressure levels for a $1 / 7^{\text {th }}$ scale $\mathrm{AH}-1$ model at a distance of 1000 rotor radii and at a position ahead of and below the helicopter on the port side [75] 


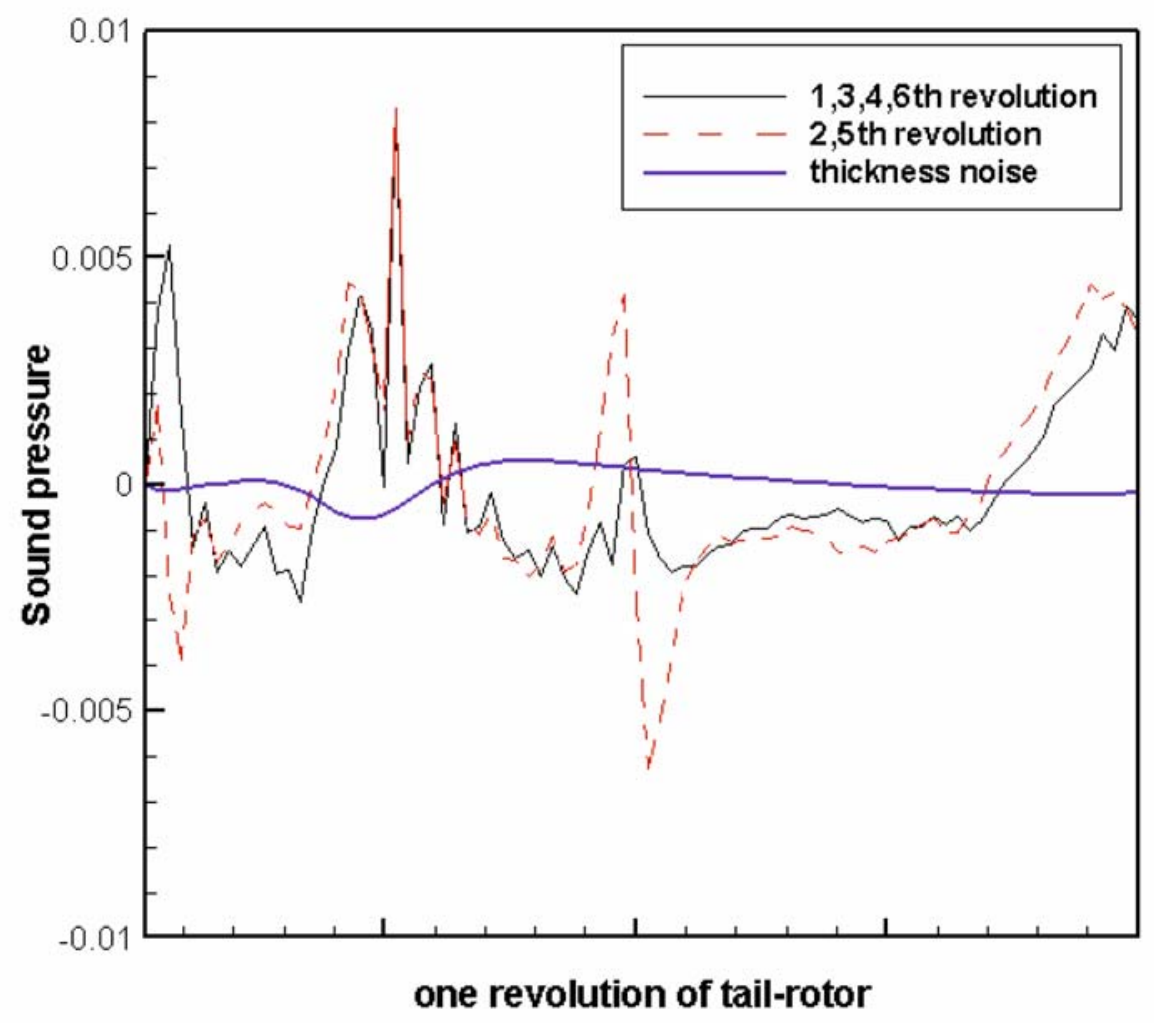

Figure 42 Computed tail rotor sound pressure levels for a $1 / 7^{\text {th }}$ scale AH-1 model at a distance of 1000 rotor radii and at a position ahead of and below the helicopter on the starboard side [75] 


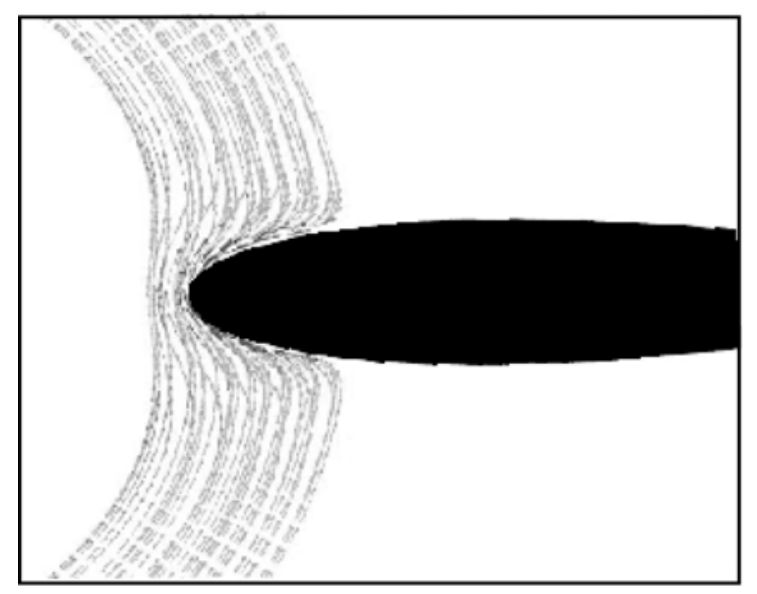

(a)

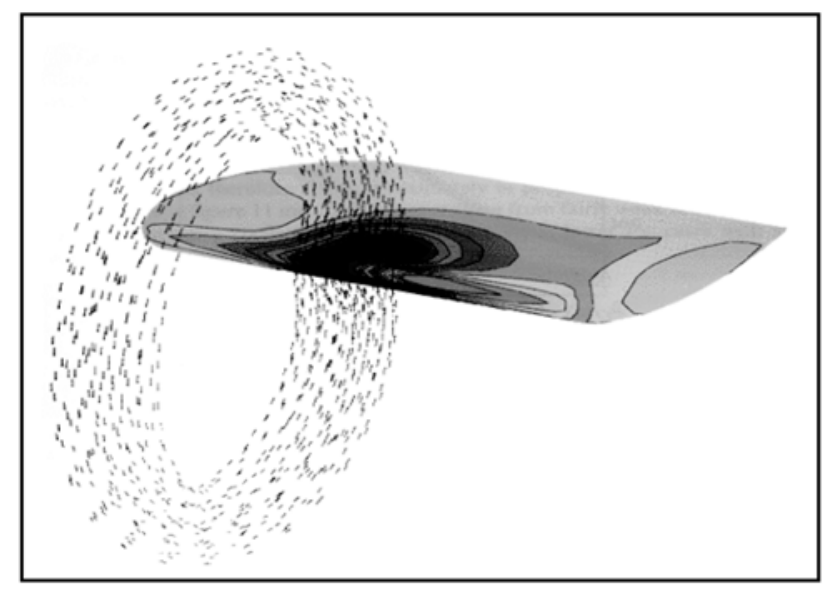

(b)

Figure 43. Results for inviscid computation of blade penetration into the core of a vortex ring, showing (a) vorticity vectors and (b) difference between pressure field and potential flow pressure field on blade surface [77]. 


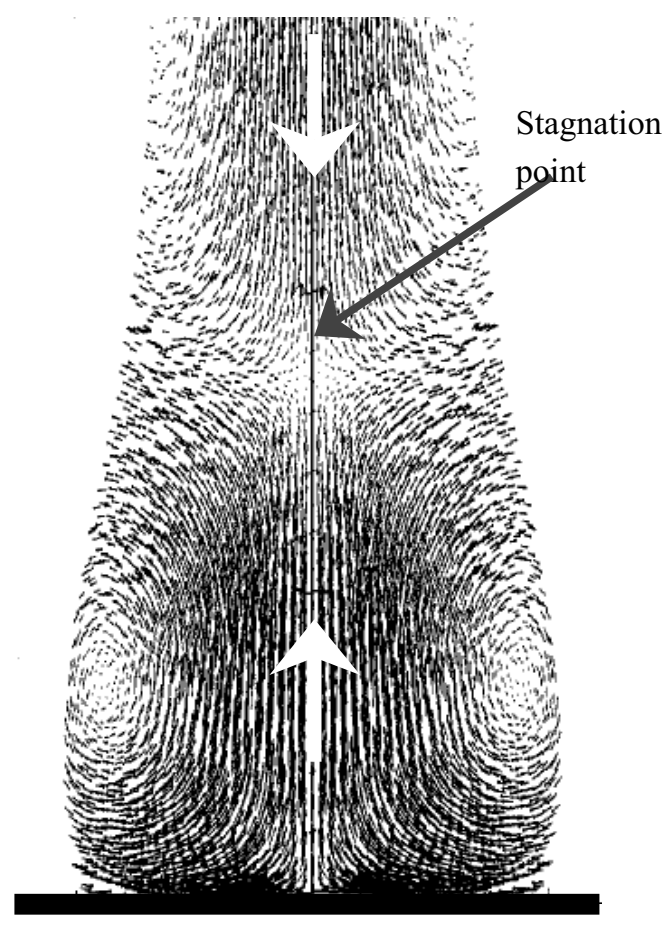

Figure 44. Axisymmetric computation showing reverse flow on upper side of vortex following impulsive cutting of a subcritical vortex $(A=0.51)$ [50]. White arrows indicate flow direction. 


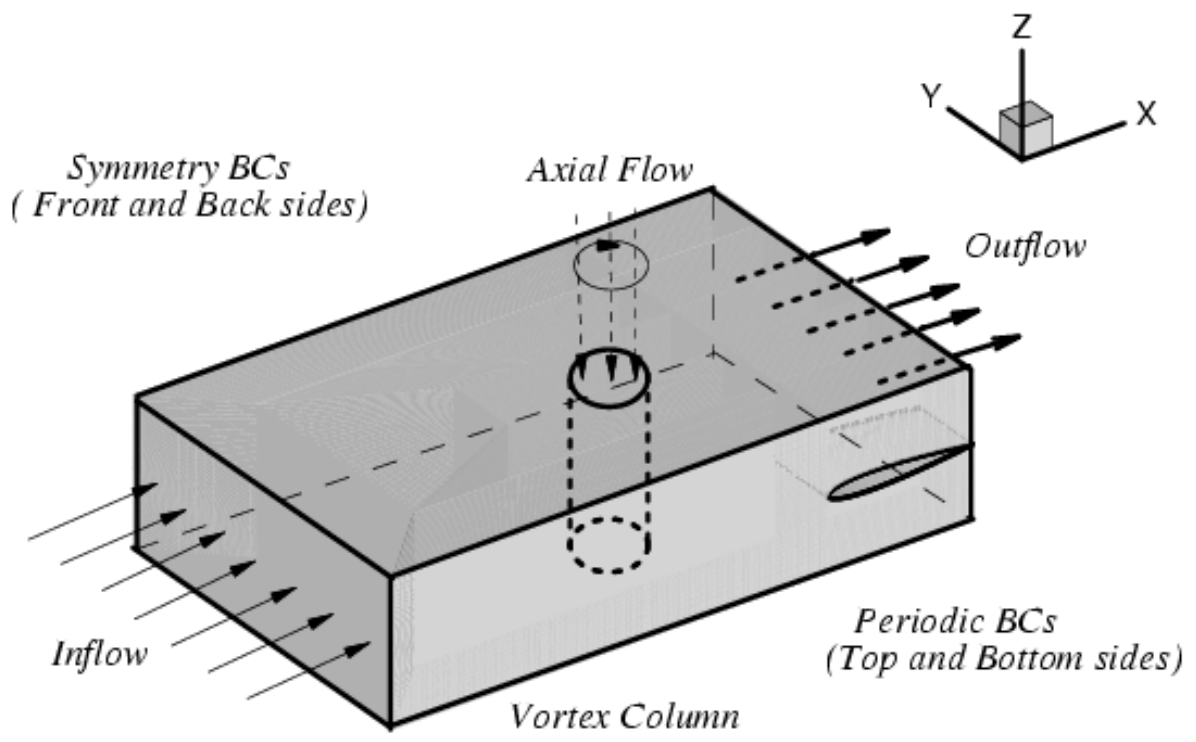

Figure 45. Boundary conditions on the computational domain for viscous vortex cutting computations [84]. 

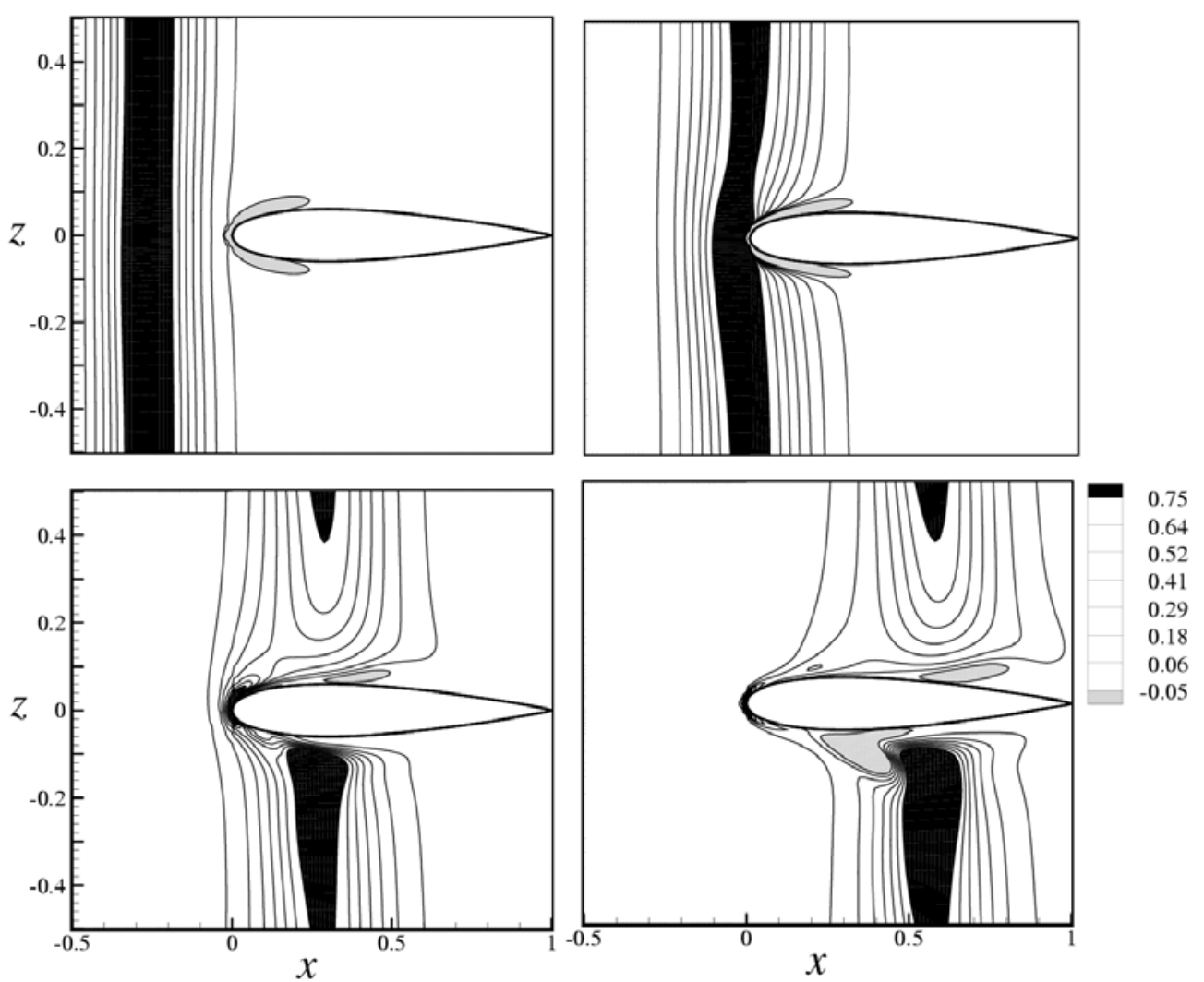

Figure 46. Contour plots of $\omega_{z}$ in $x-z$ plane for cutting of a supercritical vortex by a blade ( $A=3.8, I=4.0, R e_{v}=488$, $T=0.78)[84]$. 


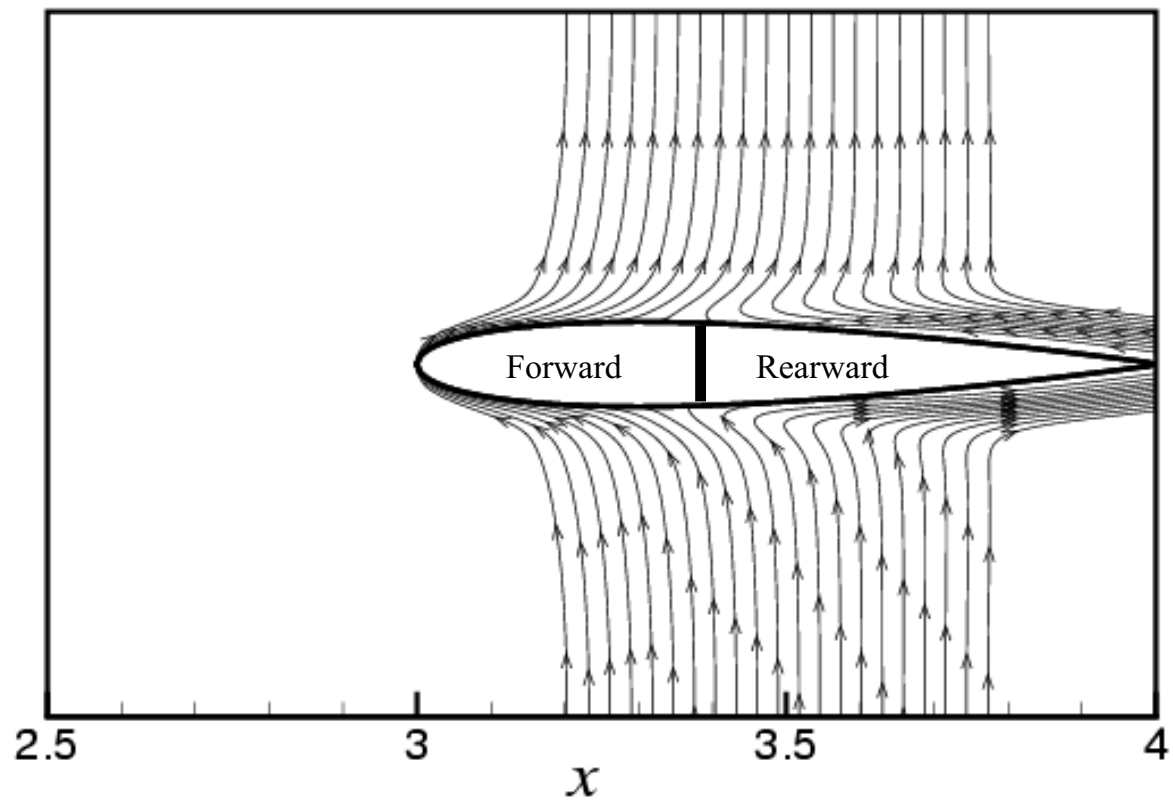

Figure 47. Vortex lines in the $x-z$ plane for a vortex with the same parameter values as in Figure 10, but with $A=0$, showing deflection of some vortex lines forward to wrap around the blade leading edge and reconnection of other vortex lines to those in the blade boundary layer, eventually leading into the blade wake [84]. 


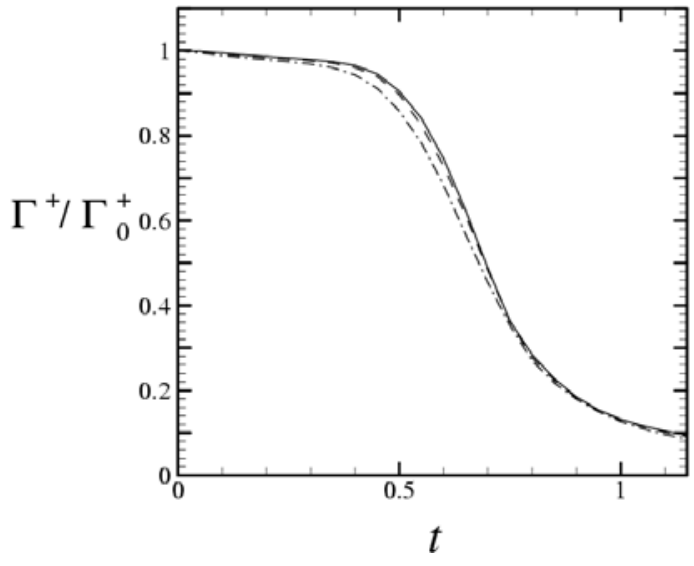

(a)

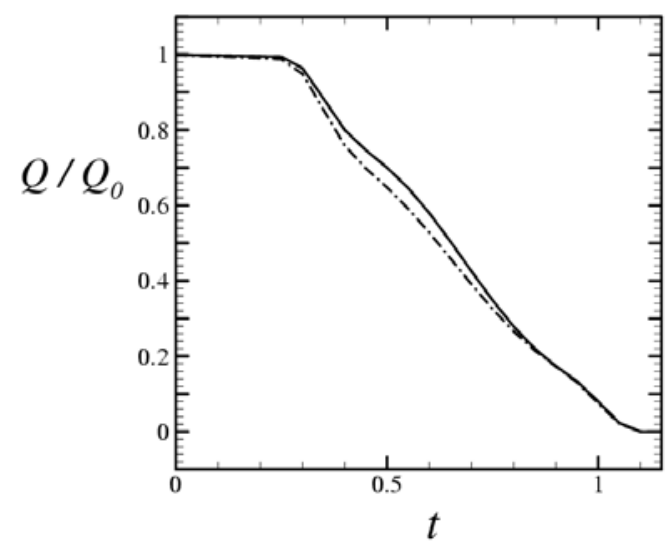

(b)

Figure 48. Effect of Reynolds number on (a) positive circulation and (b) flow rate in the $z$ (axial) direction in the blade centerplane $z=0$, nondimensinalized by their initial values, plotted for cases with vortex Reynolds number $\operatorname{Re}_{v}=488$ (solid curve), 245 (dashed curve), and 73 (dashed-dotted curve) (with other parameters the same as in Figure 10) [84]. 


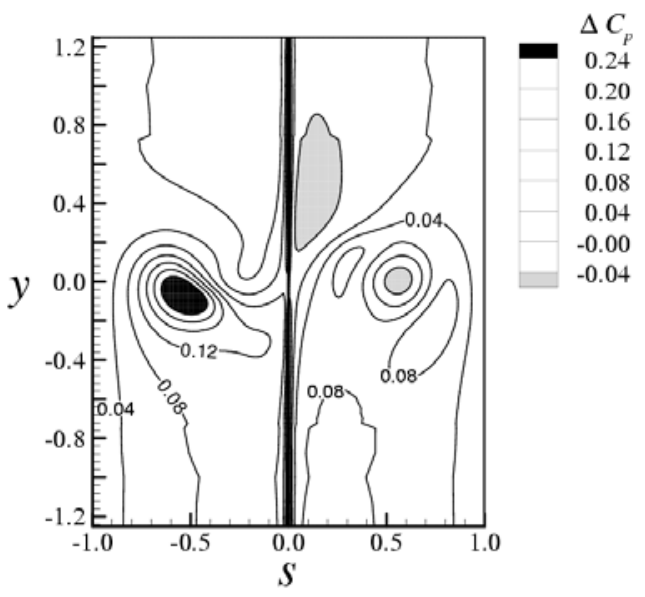

(a)

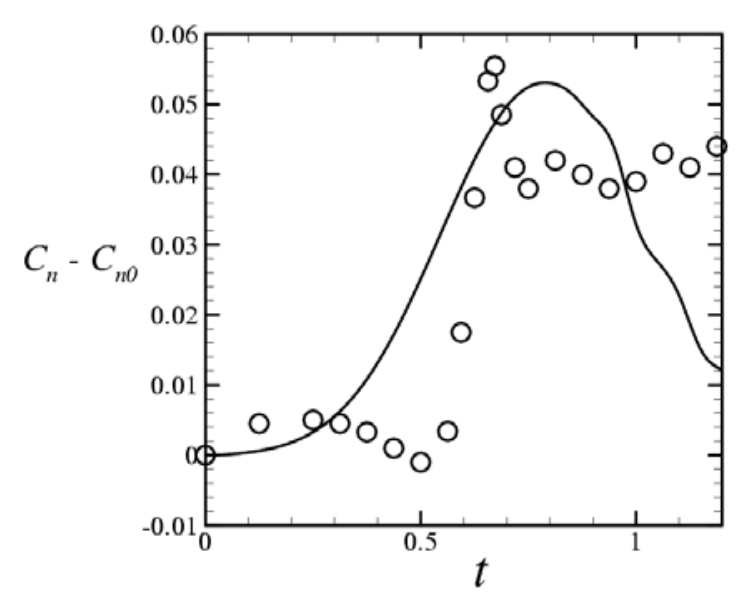

(b)

Figure 49. (a) Contour lines of the difference between the pressure coefficient with axial vortex flow (with same parameter values as in Figure 47) and with no axial vortex flow as a function of arc length around the blade surface.

(b) Comparison of the computed normal force coefficient within a slice at $\mathrm{y}=0$ for the computations (solid curve) and the experimental data of Wang et al. (2002) (circles) for a case with $A=1.8, I=41, T=0.42$, and $R e_{v}=44$ [84]. 


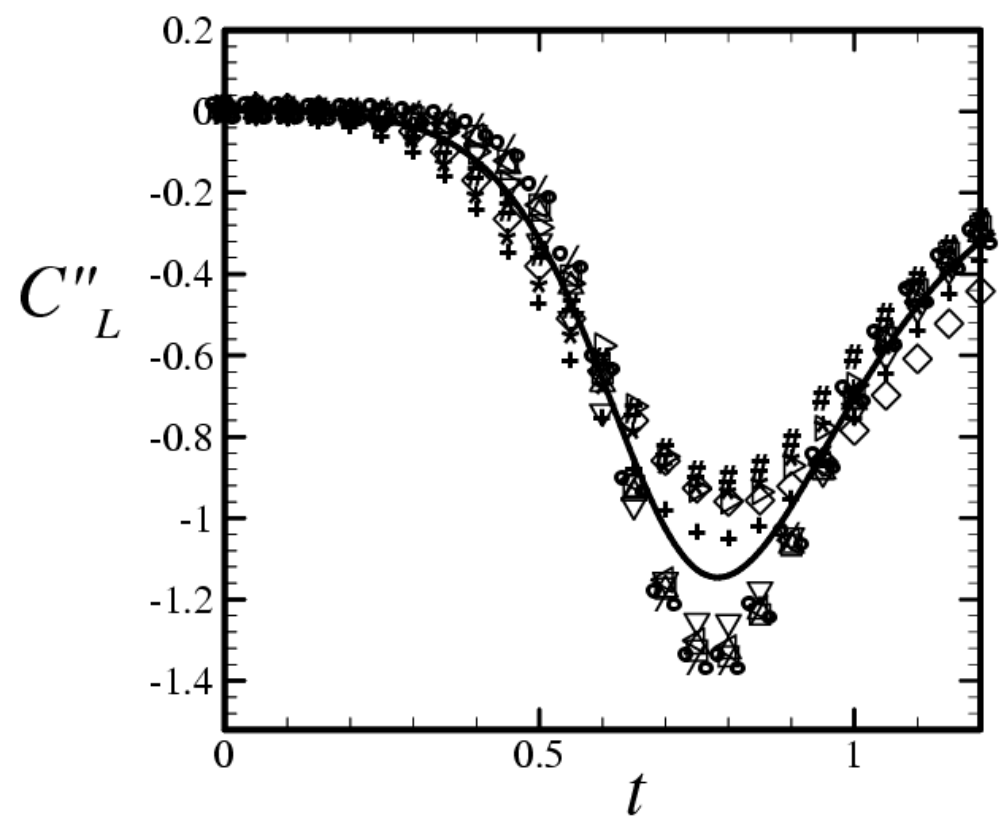

\begin{tabular}{|c|c|c|c|c|c|}
\hline Symbol & $I$ & $T$ & & $A$ & $\operatorname{Re}_{B}$ \\
\hline+ & 4.0 & 0.78 & 488 & 0.51 & 2000 \\
\hline$\#$ & 10 & 0.56 & 45 & 0.85 & 1000 \\
\hline$*$ & 41 & 0.42 & 44 & 1.8 & 1000 \\
\hline$\square$ & 4.0 & 0.78 & 488 & 3.8 & 2000 \\
\hline$\Delta$ & 4.0 & 0.78 & 245 & 3.8 & 1000 \\
\hline$\nabla$ & 4.0 & 0.78 & 73 & 3.8 & 300 \\
\hline$\triangleleft$ & 4.0 & 0.78 & 488 & 3.0 & 2000 \\
\hline$\triangleright$ & 16 & 0.78 & 488 & 3.0 & 2000 \\
\hline$\diamond$ & 33 & 0.78 & 488 & 3.0 & 2000 \\
\hline$\%$ & 4.0 & 0.39 & 488 & 3.8 & 2000 \\
\hline
\end{tabular}

Figure 50. Correlation for lift coefficient on the blade from the computational results as a function of dimensionless time [84]. 